\title{
Functional Integral Construction of the Thirring model: axioms verification and massless limit
}

\author{
G. Benfatto P. Falco V. Mastropietro \\ Dipartimento di Matematica, Università di Roma "Tor Vergata" \\ via della Ricerca Scientifica, I-00133, Roma
}

\begin{abstract}
We construct a QFT for the Thirring model for any value of the mass in a functional integral approach, by proving that a set of Grassmann integrals converges, as the cutoffs are removed and for a proper choice of the bare parameters, to a set of Schwinger functions verifying the Osterwalder-Schrader axioms. The corresponding Ward Identities have anomalies which are not linear in the coupling and which violate the anomaly non-renormalization property. Additional anomalies are present in the closed equation for the interacting propagator, obtained by combining a Schwinger-Dyson equation with Ward Identities.
\end{abstract}

\section{$1 \quad$ Introduction and Main result}

\subsection{Historical Introduction}

Proposed by Thirring [T] half a century ago, the Thirring model is a Quantum Field Theory (QFT) of a spinor field in a two dimensional space-time, with a self interaction of the form $(\lambda / 4) \int d \mathbf{x}\left(\bar{\psi}_{\mathbf{x}} \gamma^{\mu} \psi_{\mathbf{x}}\right)^{2}$. The interest of such a model, witnessed by the enormous number of papers devoted to it, is mainly due to the fact that it has a non trivial behavior, similar to the one of more realistic models, but at the same time it is simple enough to be in principle accessible to an analytic investigation. Hence the validity of several properties of QFT models, which in general can be verified at most by perturbative expansions, can be checked in principle in the Thirring model at a non-perturbative level. The Thirring model has been studied along the years following different approaches and we will recall here briefly the main achievements.

Exact approach. After a certain number of "solutions" of the model fell into disrepute after inconsistences were encountered, Johnson $[\mathrm{J}]$ was able to derive, in the massless case, an exact expression for the two point function; if $\left\langle T\left(\psi_{\mathbf{x}} \bar{\psi}_{\mathbf{0}}\right)\right\rangle$ is the two-point function in the Minkowski space, he found that 
$\left\langle T\left(\psi_{\mathbf{x}} \bar{\psi}_{\mathbf{0}}\right)\right\rangle=i\left(\bar{\gamma}^{\mu} \partial_{\mu}\right)^{-1}\left(|\mathbf{x}| / x_{0}\right)^{-\eta_{z}}$, where $\eta_{z}=2(\lambda / 4 \pi)^{2}\left[1-(\lambda / 4 \pi)^{2}\right]^{-1}, \bar{\gamma}_{\mu}$ are the Minkowski gamma matrices and $x_{0}$ is an arbitrary constant with the dimension of a length. This result was followed shortly $[\mathrm{K}]$ by the general $n$-point function at non-coinciding points. The Johnson solution, based on operator techniques, is essentially a self-consistency argument: a number of reasonable requirements on the correlations is assumed from which their explicit expression can be determined. The first assumpion is the validity of Ward-Takahashi Identities (WTi) of the form

$$
\begin{aligned}
& i \partial_{\mu}\left\langle T\left(j_{\mathbf{z}}^{\mu} \psi_{\mathbf{x}} \bar{\psi}_{\mathbf{y}}\right)\right\rangle=a[\delta(\mathbf{z}-\mathbf{x})-\delta(\mathbf{z}-\mathbf{y})] i\left\langle T\left(\psi_{\mathbf{x}} \bar{\psi}_{\mathbf{y}}\right)\right\rangle, \\
& i \partial_{\mu}\left\langle T\left(j_{\mathbf{z}}^{\mu, 5} \psi_{\mathbf{x}} \bar{\psi}_{\mathbf{y}}\right)\right\rangle=\bar{a}[\delta(\mathbf{z}-\mathbf{x})-\delta(\mathbf{z}-\mathbf{y})] \gamma^{5} i\left\langle T\left(\psi_{\mathbf{x}} \bar{\psi}_{\mathbf{y}}\right)\right\rangle,
\end{aligned}
$$

where the current $j_{\mathbf{x}}^{\mu}$ and pseudocurrent $j_{\mathbf{x}}^{\mu, 5}$ are operators, formally defined respectively as $\bar{\psi}_{\mathbf{x}} \bar{\gamma}^{\mu} \psi_{\mathbf{x}}$ and $\bar{\psi}_{\mathbf{x}} \bar{\gamma}^{\mu} \gamma^{5} \psi_{\mathbf{x}}$, and the coefficients $a^{-1}-1$ and $\bar{a}^{-1}-1$ are called anomalies; they would vanish in the naive WTi which one would expect from the classical conservation laws, see for instance [A1]. The second assumption was the validity of Schwinger-Dyson equations (the analogue of the equations of motion), and, combining them with the WTi, closed equations for the $n$-point functions were found; from them an explicit expression for the $n$-point function at distinct points was derived and, by a self-consistency argument, the following explicit values for the anomalies:

$$
a^{-1}=1-\frac{\lambda}{4 \pi} \quad \bar{a}^{-1}=1+\frac{\lambda}{4 \pi} .
$$

The anomalies are then linear in the coupling, that is no higher orders contributions are present; this property is called anomaly non-renormalization or Adler-Bardeen theorem, and it holds, as a statement valid at all orders in perturbation theory and with suitable regularizations $[\mathrm{AB}]$, in realistic models like $Q E D$ or the Electroweak model in $d=4$ (in the last model it plays a crucial role in the proof of its perturbative renormalizability). The validity of (2) in the Thirring model is particularly significant, as it has been considered $[\mathrm{GR}]$ as a non-perturbative verification of the perturbative analysis of $[\mathrm{AB}]$ adapted to this case; however the applicability of the $[\mathrm{AB}]$ analysis to the Thirring model has been also questioned $[\mathrm{AF}]$. Another remarkable relation found in the exact analysis in $[\mathrm{J}]$ is

$$
\eta_{z}=\frac{\lambda}{2 \pi}(a-\bar{a})
$$

relating the anomalous exponent of the two point function with the anomalies; it is an immediate consequence of the the closed equation for the two point functions obtained by inserting the WTi (1) in the Schwinger-Dyson equation. The outcome of this exact analysis is an explicit expression for the $n$-point function at non-coinciding points and for the WTi. However, as stressed by Wigthmann [W], the procedure is not satisfactory from a 
mathematical point of view, as it involves several formal manipulations of diverging quantities; even the meaning of the basic equation (1) is unclear as the averages in the l.h.s. and r.h.s. has to be (formally) divided by a vanishing constant to be not identically vanishing.

Axiomatic approach. The Johnson analysis still left as an open problem the rigorous construction of a QFT corresponding to the massless Thirring model. Wigthmann [W] proposed to construct the massless Thirring model following an axiomatic approach; one can start directly from the explicit expressions of the $n$-point functions at non-coinciding points derived in $[\mathrm{J}],[\mathrm{K}]$ (forgetting how they were derived) and try to verify the axioms necessary for the reconstruction theorem. Indeed all axioms can be easily verified except positive definiteness, which was proved later on in [DFZ] and [CRW]; the idea was to define certain field operators, depending on a certain number of parameters, whose expectations verify the positivity property by construction and such that their $n$-point functions coincide, for a suitable choice of the parameters, with the expression found in $[\mathrm{J}],[\mathrm{K}]$. As the axioms are verified by the $n$-point functions of $[\mathrm{J}],[\mathrm{K}]$, a rigorous construction of a QFT corresponding to massless Thirring model is then obtained. Note however that the fermionic mass cannot be included in this approach; moreover quadratic fermionic operators at coinciding points, like $j_{\mathbf{x}}^{\mu}$ or $j^{\mu, 5}$ cannot be considered, hence the WTi (1) cannot be rigorously derived.

Perturbative approach. The massive case is much less understood; Coleman $[\mathrm{C}]$ considered a perturbation expansion in the mass showing that it was order by order coinciding with the expansion of the Sine-Gordon model, if suitable identification of parameters is done; however an explicit expression for the n-point functions was not obtained, hence a QFT corresponding to the massive Thirring model has never been constructed.

Bosonic functional integral approach. If the coupling $\lambda$ is positive, the partition function and the generating functional of the massless (Euclidean) Thirring model can be written as bosonic functional integrals [FGS] by a Hubbard-Stratonovich transformation; one can then integrate the fermion variables and it turns out that the partition function of the Thirring model can be written as

$$
\int P(d A) \frac{\operatorname{det}\left(\gamma_{\mu}\left[\partial_{\mu}+A_{\mu}\right]\right)}{\operatorname{det}\left(\gamma_{\mu} \partial_{\mu}\right)}
$$

where $A_{\mu, \mathbf{x}}$ is a two-dimensional Gaussian field with covariance $\left\langle A_{\mu, \mathbf{x}} A_{\nu, \mathbf{y}}\right\rangle=$ $\lambda \delta_{\mu, \nu} \delta(\mathbf{x}-\mathbf{y})$ and $\gamma_{\mu}$ are the Euclidean gamma matrices. A similar expression holds for the generating functional. It is well known $[\mathrm{S}]$ that, under suitably regularity conditions over $A, \log \operatorname{det}\left(\gamma_{\mu} \partial_{\mu}+\gamma_{\mu} A_{\mu}\right)-\log \operatorname{det}\left(\gamma_{\mu} \partial_{\mu}\right)$ is quadratic in $A$; by replacing the determinant with a quadratic exponential, one then gets an explicitly solvable integral, from which the n-point functions 
can be derived. As stressed in [FGS], in this way one gets in a very simple way the results of the exact approach found in $[\mathrm{J}]$ and $[\mathrm{K}]$. In particular the relation (3) for the two point critical index $\eta_{z}$ is verified and the anomalies (2) can be easily computed. If a dimensional regularization is adopted, one finds $a=1$ and $\bar{a}^{-1}-1=\lambda /(2 \pi)$, while with a momentum regularization (2) holds; in both cases the anomaly non-renormalization holds. Of course in the above derivation an approximation is implicit; the logarithm of the fermionic determinant in (4) is given by a quadratic expression only if $A$ is sufficiently regular, but the integral is over all possible fields $A$, hence one is neglecting the contributions of the irregular fields and there is no guarantee at all that such contribution is negligible. This approximation is usually supported by the fact that one gets in this way the same results found in $[\mathrm{J}]$ and $[\mathrm{K}]$.

Fermionic functional integral approach. This is the approach we will follow in this paper. The generating functional for the Euclidean Thirring model is the following Grassmann integral (see below for a more precise definition)

$$
e^{\mathcal{W}(\phi, J)}=\frac{1}{\mathcal{N}} \int P_{N}(d \psi) e^{\int d \mathbf{x}\left[-\frac{\lambda}{4}\left(\bar{\psi}_{\mathbf{x}} \gamma^{\mu} \psi_{\mathbf{x}}\right)^{2}+J_{\mu, \mathbf{x}} \bar{\psi}_{\mathbf{x}} \gamma_{\mu} \psi_{\mathbf{x}}+\frac{\phi_{\mathbf{x}} \bar{\psi}_{\mathbf{x}}}{\sqrt{Z_{N}}+\frac{\bar{\phi}_{\mathbf{x}} \psi_{\mathbf{X}}}{\sqrt{Z_{N}}}},\right.}
$$

where $\mathcal{N}$ is a normalization constant, $\phi, J$ are external fields, $Z_{N}$ is the wave function renormalization, $\psi_{\mathbf{x}}, \bar{\psi}_{\mathbf{x}}$ are Grassmann variables, $P_{N}(d \psi)$ is the fermionic integration corresponding to a fermionic propagator with mass $\mu_{N}$ and a (smooth) momentum ultraviolet cut-off $\gamma^{N}$, with $\gamma>1$. Note that the averages of $\bar{\psi}_{\mathbf{x}} \gamma^{\mu} \gamma^{5} \psi_{\mathbf{x}}$ can be obtained by the derivatives with respect to $J_{\mu}$, using the relation $\bar{\psi}_{\mathbf{x}} \gamma^{\mu} \gamma^{5} \psi_{\mathbf{x}}=-i \varepsilon_{\mu, \nu} \bar{\psi}_{\mathbf{x}} \gamma^{\mu} \psi_{\mathbf{x}}$ with $\varepsilon_{\mu, \nu}=-\varepsilon_{\nu, \mu}$ and $\varepsilon_{1,0}=1$. When $J=\phi=0$ and $\mu_{N}=0$ the r.h.s. of (5) coincides with (4) (if $\lambda$ is positive) in the limit $N \rightarrow \infty$.

We will show that, by properly choosing the bare wave function renormalization $Z_{N}$ and the bare mass $\mu_{N}$, the Schwinger functions at noncoinciding points obtained from (5) converge, for $N \rightarrow \infty$, to a set of functions verifying the Osterwalder-Schrader axioms [OS2] for an Euclidean QFT. These functions depend on three parameters, the physical mass, the physical wave function renormalization and the physical coupling, but they are independent on the way the ultraviolet cutoff is explicitly realized. On the contrary, the relation between the physical and the bare parameters depends on the details of the ultraviolet cutoff.

In this way we have obtained for the first time a construction of a QFT for the Thirring model for any value of the (physical) mass. Moreover, even if in the massless case other constructions were known, we find in any case interesting to reach a complete construction of the Thirring model relying only on a functional integral approach, which could be the only possible one at higher dimensions or for more realistic models. 
The analysis of the functional integral (5) is performed by a multiscale analysis using a (Wilsonian) Renormalization Group approach as in [G]. After each iteration step an effective theory with new couplings, mass, wave function and current renormalizations is obtained. The effective parameters obey to a recursive equation called Beta function, and a major technical problem is that this iterative procedure can be controlled only by proving non trivial cancellations in the Beta function. Such cancellations are established by suitable WTi valid at each scale and reflecting the symmetries of the formal action; contrary to the WTi formally valid when all cutoffs are removed, they have corrections due to the cutoffs introduced for performing the multiscale integration. The crucial role of WTi in the construction of the theory is a feature that the functional integral (5) shares with realistic models like $Q E D$ or the Electroweak theory in $d=4$, requiring WTi even to prove the perturbative renormalizability, which is absent in the models previously rigorously constructed by functional integral methods, like the massive Yukawa model [Le] or the massive Gross-Neveu model [GK, FMRS]. From the functional integral (5) we obtain, for $N \rightarrow \infty$ and in the massless limit, WTi of the same form as the one postulated in $[\mathrm{J}]$ :

$$
\begin{aligned}
& \partial_{\mu}\left\langle\bar{\psi}_{\mathbf{z}} \gamma^{\mu} \psi_{\mathbf{z}} ; \psi_{\mathbf{x}} \bar{\psi}_{\mathbf{y}}\right\rangle=a[\delta(\mathbf{z}-\mathbf{x})-\delta(\mathbf{z}-\mathbf{y})]\left\langle\psi_{\mathbf{x}} \bar{\psi}_{\mathbf{y}}\right\rangle \\
& \partial_{\mu}\left\langle\bar{\psi}_{\mathbf{z}} \gamma^{\mu} \gamma^{5} \psi_{\mathbf{z}} ; \psi_{\mathbf{x}} \bar{\psi}_{\mathbf{y}}\right\rangle=\bar{a}[\delta(\mathbf{z}-\mathbf{x})-\delta(\mathbf{z}-\mathbf{y})] \gamma^{5}\left\langle\psi_{\mathbf{x}} \bar{\psi}_{\mathbf{y}}\right\rangle
\end{aligned}
$$

where $\left\langle\psi_{\mathbf{x}} \bar{\psi}_{\mathbf{y}}\right\rangle=\left.\lim _{N \rightarrow \infty} \frac{\partial^{2}}{\partial \phi_{\mathbf{x}} \partial \phi_{\mathbf{y}}} \mathcal{W}\right|_{0}$ (similar definitions hold for the other averages); however the anomaly coefficients in (6) are given by the following expression

$$
a^{-1}=1-\frac{\lambda}{4 \pi}+c_{+} \lambda^{2}+O\left(\lambda^{3}\right) \quad, \quad \bar{a}^{-1}=1+\frac{\lambda}{4 \pi}+c_{+} \lambda^{2}+O\left(\lambda^{3}\right),
$$

where $c_{+}$is a non-vanishing constant, (its explicit value is calculated in Appendix B). The anomaly coefficients are not linear in the bare coupling (the anomaly non-renormalization is violated), contrary to what happens in the values (2), found in the exact approach. Indeed the regularizations used in the exact solution are different with respect to the ones used in the functional integral approach, and it is not too surprising to get different properties (despite often is guessed that the same results should be obtained by the two approaches). In particular, the constant $c_{+}$, not only is different from 0 , but even depends on the way the ultraviolet cutoff is realized. The difference of (7) with respect to (2) also implies that the approximation in (4) of the determinant with a quadratic exponential does not lead to correct results, at least if a momentum regularization is used.

In (5) a bare wave function $Z_{N}$ for the fermionic fields has been introduced, to be fixed so that the "physical" renormalization has a fixed value at the "laboratory scale"; analogously we can introduce a (finite) bare charge also for the current, defining it as $\xi \bar{\psi} \gamma^{\mu} \psi$. A physically meaningful choice 
for $\xi$ could be $\xi=a^{-1}$, implying that the current has no anomalies; this choice fix the renormalization even of the pseudocurrent (remember that $\bar{\psi} \gamma^{\mu} \gamma^{5} \psi=i \varepsilon_{\mu, \nu} \bar{\psi} \gamma^{\mu} \psi$ ), which has then still anomalies.

Note that (7) is not in contrast to the Adler-Bardeen [AB] analysis, as they consider a boson-fermion interaction with a massive boson, which corresponds to require a non local current-current interaction. If we replace in (5) the local current-current interaction with a non local short ranged one, still a WTi like (6) is found for $N \rightarrow \infty$, but the anomalies are linear in $\lambda$ and identical to the ones found in the exact approach, that is they are given by (2) instead of $(7)$, see $[\mathrm{M}]$.

Finally we will show that a closed equation for the 2-point function is indeed valid starting from the functional integral (5); it is however different with respect to the one postulated in $[\mathrm{J}]$ (which was the natural one obtained inserting the WTi in the Schwinger-Dyson equation) for the presence of additional anomalies. As a consequence, we get a relation between the critical index of the two point function and the anomalies different with respect to (3), namely

$$
\eta_{z}=\frac{\lambda}{4 \pi}(a-\bar{a})\left[1-c_{0} \lambda+O\left(\lambda^{2}\right)\right]
$$

with $c_{0}>0$ nonvanishing. This additional anomalies says that the closed equation for the 2-point function is not simply obtained inserting the WTi in the Schwinger-Dyson equation.

In the rest of this section we will define more precisely our regularized functional integral and we state our main results. We will find more convenient, from the point of view of the notation, to introduce the Weyl spinors $\psi_{\mathbf{x}, \omega}^{ \pm}$, with $\omega= \pm$, such that $\psi_{\mathbf{x}}=\left(\psi_{\mathbf{x},+}^{-}, \psi_{\mathbf{x},-}^{-}\right), \bar{\psi} \equiv \psi^{\dagger} \gamma^{0}$ and $\psi_{\mathbf{x}}^{+}=\left(\psi_{\mathbf{x},+}^{+}, \psi_{\mathbf{x},-}^{+}\right)$; the $\gamma^{\prime}$ 's matrices are explicitly given by

$$
\gamma^{0}=\left(\begin{array}{cc}
0 & 1 \\
1 & 0
\end{array}\right), \quad \gamma^{1}=\left(\begin{array}{cc}
0 & -i \\
i & 0
\end{array}\right), \quad \gamma^{5}=-i \gamma^{0} \gamma^{1}=\left(\begin{array}{cc}
1 & 0 \\
0 & -1
\end{array}\right) .
$$

\subsection{Thirring model with cutoff}

We introduce in $\Lambda=[-L / 2, L / 2] \times[-L / 2, L / 2]$ a lattice $\Lambda_{a}$ whose sites are given by the space-time points $\mathbf{x}=\left(x, x_{0}\right)=\left(n a, n_{0} a\right)$, with $L / 2 a$ integer and $n, n_{0}=-L / 2 a, \ldots, L / 2 a-1$. We also consider the set $\mathcal{D}_{a}$ of spacetime momenta $\mathbf{k}=\left(k, k_{0}\right)$, with $k=\left(m+\frac{1}{2}\right) \frac{2 \pi}{L}$ and $k_{0}=\left(m_{0}+\frac{1}{2}\right) \frac{2 \pi}{L}$ and $m, m_{0}=-L / 2 a, \ldots, L / 2 a-1$. In order to introduce an ultraviolet and an infrared cutoff, we fix a number $\gamma>1$, a positive integer $N$ and a negative integer $h$; then we define the function $C_{h, N}^{-1}(\mathbf{k})$ in the following way; let $\chi_{0} \in C^{\infty}\left(\mathbb{R}_{+}\right)$be a non-negative, non-increasing smooth function such that

$$
\chi_{0}(t) \stackrel{\text { def }}{=}\left\{\begin{array}{rr}
1 & \text { if } 0 \leq t \leq 1 \\
0 & \text { if } t \geq \gamma_{0}
\end{array}\right.
$$


for a fixed choice of $\gamma_{0}: 1<\gamma_{0} \leq \gamma$; then we define, for any $h \leq j \leq N$,

$$
f_{j}(\mathbf{k}) \stackrel{\text { def }}{=} \chi_{0}\left(\gamma^{-j}|\mathbf{k}|\right)-\chi_{0}\left(\gamma^{-j+1}|\mathbf{k}|\right)
$$

and $C_{h, N}^{-1}(\mathbf{k})=\sum_{j=h}^{N} f_{j}(\mathbf{k})$; hence $C_{h, N}^{-1}(\mathbf{k})$ acts as a smooth cutoff for momenta $|\mathbf{k}| \geq \gamma^{N+1}$ (ultraviolet region) and $|\mathbf{k}| \leq \gamma^{h-1}$ (infrared region). It is useful for technical reasons to choose for $\chi_{0}(t)$ a Gevrey function, for example one of class 2 , that is a function such that, for any integer $n$,

$$
\left|d^{n} \chi_{0}(t) / d t^{n}\right| \leq C^{n}(n !)^{2}
$$

where $C$ is a symbol we shall use regularly in the following to denote a generic constant. With each $\mathbf{k} \in \mathcal{D}_{a}$ we associate four Grassmann variables $\left\{\widehat{\psi}_{\mathbf{k}, \omega}^{h, N] \sigma}, \sigma, \omega= \pm\right\}$, to be called field variables; we define $\mathcal{D}^{[h, N]} \stackrel{\text { def }}{=}\left\{\mathbf{k} \in \mathcal{D}_{a}: C_{h, N}^{-1}(\mathbf{k}) \neq 0\right\}$. On the finite Grassmannian algebra generated from these variables we define a linear functional $\mathrm{d} \widehat{\psi}^{[h, N]}$ (the Lebesgue measure), so that, given a monomial $\mathcal{Q}(\psi)$ in the field variables, $\int \mathrm{d} \widehat{\psi}^{[h, N]} \mathcal{Q}(\psi)=0$ except in the case $\mathcal{Q}(\psi)$ is equal to $\mathcal{Q}_{0}(\psi)=\prod_{\mathbf{k} \in \mathcal{D}^{[h, N]}} \prod_{\omega= \pm} \widehat{\psi}_{\mathbf{k}, \omega}^{[h, N]-} \widehat{\psi}_{\mathbf{k}, \omega}^{[h, N]+}$ or to one of the monomials obtained from $\mathcal{Q}_{0}(\psi)$ by a permutation of the field variables; in these cases the value of $\int \mathrm{d} \widehat{\psi}^{[h, N]} \mathcal{Q}(\psi)$ is determined by the condition $\int \mathrm{d} \widehat{\psi}^{[h, N]} \mathcal{Q}_{0}(\psi)=1$ and the anticommuting properties of the field variables.

We also define a Grassmann field on the lattice $\Lambda_{a}$ by Fourier transform, according to the following convention:

$$
\psi_{\mathbf{x}, \omega}^{[h, N] \sigma \stackrel{\text { def }}{=}} \frac{1}{L^{2}} \sum_{\mathbf{k} \in \mathcal{D}_{a}} e^{i \sigma \mathbf{k x}} \widehat{\psi}_{\mathbf{k}, \omega}^{[h, N] \sigma}, \quad \mathbf{x} \in \Lambda_{a}
$$

By the definition of $\mathcal{D}_{a}, \psi_{\mathbf{x}, \omega}^{[h, N] \sigma}$ is antiperiodic both in time and in space coordinate.

The Generating Functional of the Thirring model with cutoff is

$$
\begin{gathered}
\mathcal{W}(\varphi, J)=\log \int P_{Z_{N}}(d \psi) \exp \left\{-\lambda V\left(\sqrt{Z_{N}} \psi\right)+\right. \\
\left.+Z_{N} \sum_{\omega} \int d \mathbf{x} J_{\mathbf{x}, \omega} \psi_{\mathbf{x}, \omega}^{[h, N]+} \psi_{\mathbf{x}, \omega}^{[h, N]-}+\sum_{\omega} \int d \mathbf{x}\left[\varphi_{\mathbf{x}, \omega}^{+} \psi_{\mathbf{x}, \omega}^{[h, N]-}+\psi_{\mathbf{x}, \omega}^{[h, N]+} \varphi_{\mathbf{x}, \omega}^{-}\right]\right\}
\end{gathered}
$$

where $\int d \mathbf{x}$ is a short hand notation for $a^{2} \sum_{\mathbf{x} \in \Lambda_{a}}$,

$$
\begin{gathered}
P_{Z_{N}}(d \psi) \stackrel{\text { def }}{=} \mathrm{d} \widehat{\psi}^{[h, N]} \cdot \prod_{\mathbf{k} \in \mathcal{D}^{[h, N]}}\left[L^{-4} Z_{N}^{2} \mid\left(-|\mathbf{k}|^{2}-\mu_{N}^{2}\right) C_{h, N}^{2}(\mathbf{k})\right]^{-1} . \\
\exp \left\{-Z_{N} \frac{1}{L^{2}} \sum_{\omega, \omega^{\prime}= \pm} \sum_{\mathbf{k} \in \mathcal{D}^{[h, N]}} \frac{T_{\omega, \omega^{\prime}}(\mathbf{k})}{C_{h, N}^{-1}(\mathbf{k})} \widehat{\psi}_{\mathbf{k}, \omega}^{[h, N]+} \widehat{\psi}_{\mathbf{k}, \omega^{\prime}}^{[h, N]-}\right\}, \\
T_{\omega, \omega^{\prime}}(k) \stackrel{\text { def }}{=}\left(\begin{array}{cc}
D_{+}(\mathbf{k}) & \mu_{N} \\
\mu_{N} & D_{-}(\mathbf{k})
\end{array}\right)_{\omega, \omega^{\prime}} ; \quad D_{\omega}(\mathbf{k}) \stackrel{\text { def }}{=}-i k_{0}+\omega k_{1},
\end{gathered}
$$




$$
V(\psi) \stackrel{\text { def }}{=} \frac{1}{2} \sum_{\omega= \pm} \int d \mathbf{x} \widehat{\psi}_{\mathbf{x}, \omega}^{[h, N]+} \widehat{\psi}_{\mathbf{x}, \omega}^{[h, N]-} \widehat{\psi}_{\mathbf{x},-\omega}^{[h, N]+} \widehat{\psi}_{\mathbf{x},-\omega}^{[h, N]-}
$$

and $\left\{J_{\mathbf{x}, \omega}\right\}_{\mathbf{x}, \omega}$ are commuting variables, while $\left\{\varphi_{\mathbf{x}, \omega}^{\sigma}\right\}_{\mathbf{x}, \omega, \sigma}$ are anticommuting. $\left\{J_{\mathbf{x}, \omega}\right\}_{\mathbf{x}, \omega}$ and $\left\{\varphi_{\mathbf{x}, \omega}^{\sigma}\right\}_{\mathbf{x}, \omega, \sigma}$ are the external field variables.

Remark. It is immediate to check that (13) coincides with (5), if the notational conventions adopted at the end of $\$ 1.1$. are used and up to the trivial rescaling $\psi \rightarrow \sqrt{Z} \psi$ of the Grassmann variables. Note also that the continuum regularization we have introduced is very suitable to derive WTi and SDe ; its main disadvantage is that the positive definiteness property is not automatically ensured; such a property will be recovered indirectly later by introducing a different regularization preserving positive definiteness and such that, by a proper choice of the bare parameters, the Schwinger functions in the limit of removed cutoffs are coinciding.

Setting $\underline{\mathbf{x}} \stackrel{\text { def }}{=} \mathbf{x}_{1}, \ldots, \mathbf{x}_{n}$, and $\underline{\mathbf{y}} \stackrel{\text { def }}{=} \mathbf{y}_{1}, \ldots, \mathbf{y}_{m}$, for any given choice of the labels $\underline{\underline{\sigma}} \stackrel{\text { def }}{=}\left(\sigma_{1} \ldots, \sigma_{m}\right)$, $\underline{\underline{\omega}} \stackrel{\text { def }}{=}\left(\omega_{1} \ldots, \omega_{n}\right)$ and $\underline{\varepsilon} \stackrel{\text { def }}{=}\left(\varepsilon_{1} \ldots, \varepsilon_{n}\right)$, the Schwinger functions are defined as

$$
S_{\underline{\boldsymbol{\sigma}} ; \underline{\underline{\omega}, \underline{\varepsilon}}}^{N, \underline{\underline{\varepsilon}},(m ; n)}(\underline{\mathbf{y}} ; \underline{\mathbf{x}})^{\stackrel{d e f}{=}} \lim _{L \rightarrow \infty} \frac{\partial^{n+m} \mathcal{W}}{\partial J_{\mathbf{y}_{1}, \sigma_{1}} \cdots \partial J_{\mathbf{y}_{m}, \sigma_{m}} \partial \varphi_{\mathbf{x}_{1}, \omega_{1}}^{\varepsilon_{1}} \cdots \partial \varphi_{\mathbf{x}_{n}, \omega_{n}}^{\varepsilon_{n}}}(0,0) .
$$

We will follow the convention that a missing label means that the corre-

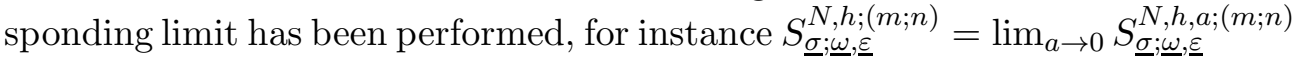
In particular, in order to shorten the notation of the most used Schwinger functions, let:

$$
\begin{aligned}
G_{\omega}^{2, N, h, a}(\mathbf{x}, \mathbf{y}) & \stackrel{\text { def }}{=} S_{\omega, \omega,(+,-)}^{N, h, a,(0 ; 2)}(\mathbf{x}, \mathbf{y}), \\
G_{\omega^{\prime}, \omega}^{2,1, N, h, a}(\mathbf{z} ; \mathbf{x}, \mathbf{y}) & \stackrel{\text { def }}{=} S_{\omega^{\prime} ; \omega, \omega,+,-}^{N, h, a,(1 ; 2)}(\mathbf{z} ; \mathbf{x}, \mathbf{y}) .
\end{aligned}
$$

We define the Fourier transforms so that, for example,

$$
\begin{aligned}
G_{\omega}^{2}(\mathbf{x}, \mathbf{y}) & \stackrel{\text { def }}{=} \int \frac{d \mathbf{k}}{(2 \pi)^{2}} e^{-i \mathbf{k}(\mathbf{x}-\mathbf{y})} \widehat{G}_{\omega}^{2}(\mathbf{k}) \\
G_{\omega^{\prime}, \omega}^{2,1}(\mathbf{z} ; \mathbf{x}, \mathbf{y}) & \stackrel{\text { def }}{=} \int \frac{d \mathbf{k} d \mathbf{p}}{(2 \pi)^{4}} e^{i \mathbf{p}(\mathbf{z}-\mathbf{y})} e^{-i \mathbf{k}(\mathbf{x}-\mathbf{y})} \widehat{G}_{\omega^{\prime}, \omega}^{2,1}(\mathbf{p}, \mathbf{k})
\end{aligned}
$$

The presence of the cutoffs makes the Schwinger functions $S_{\underline{\sigma} ; \underline{\omega},(\underline{\varepsilon})}^{N, a ;(m ; n)}(\underline{\mathbf{y}} ; \underline{\mathbf{x}})$ well defined, since the generating functional is simply a polynomial in the external field variables, for any finite $L$, and the limit $L \rightarrow \infty$ gives no problem, if $h$ is finite. Note that the lattice is introduced just to give a meaning to the Grassmann integral and it can be removed safely if $h, N$ are fixed. In $\S 2$ we will prove the following result. 
Theorem 1 Given $\lambda$ small enough and $\mu>0$, there exist functions $Z_{N} \equiv$ $Z_{N}(\lambda) \mu_{N} \equiv \mu g_{N}(\lambda)$, such that

$$
Z_{N}=\gamma^{-N \eta_{z}}\left(1+\mathrm{O}\left(\lambda^{2}\right)\right) \quad, \quad \mu_{N}=\mu \gamma^{-N \eta_{\mu}}(1+\mathrm{O}(\lambda))
$$

with $\eta_{z}=a_{z} \lambda^{2}+\mathrm{O}\left(\lambda^{4}\right), \eta_{\mu}=-a_{\mu} \lambda+\mathrm{O}\left(\lambda^{2}\right), a_{z}, a_{\mu}>0$, and the following is true.

1. The limit

$$
\lim _{N,-h, a^{-1} \rightarrow \infty} S_{\underline{\sigma} ; \underline{\omega}, \underline{\varepsilon}}^{N, h, a ;(m ; n)}(\underline{\mathbf{y}} ; \underline{\mathbf{x}})=S_{\underline{\sigma} ; \underline{\omega}, \underline{\varepsilon}}^{(m ; n)}(\underline{\mathbf{y}} ; \underline{\mathbf{x}}),
$$

exist at non coinciding points.

2. The family of functions $S_{2 n, \underline{\omega}}(\underline{\mathbf{x}})$, defined as equal to $S_{\underline{\omega}, \underline{\underline{w}}}^{(0 ; 2 n)}(\underline{\mathbf{x}})$, with $\varepsilon_{i}=+1$ for $i=1, \ldots, n$ and $\varepsilon_{i}=-1$ for $i=n+1, \ldots, 2 n$, fulfills the OSa.

3. The two point Schwinger function verifies the following bound

$$
\left|G_{\omega}^{2}(\mathbf{x}, \mathbf{y})\right| \leq \frac{C}{|\mathbf{x}-\mathbf{y}|^{1+\eta_{z}}} e^{-c \sqrt{\kappa \mu^{1+\eta_{\mu}^{\prime}}|\mathbf{x}-\mathbf{y}|}},
$$

with $\eta_{\mu}^{\prime}=a_{\mu} \lambda+O\left(\lambda^{2}\right)$. Moreover $G_{\omega}^{2}(\mathbf{x}, \mathbf{y})$ is singular for $\mathbf{x} \rightarrow \mathbf{y}$ and it diverges as $|\mathbf{x}-\mathbf{y}|^{-1-\eta_{z}}$.

4. In the massless limit $\mu \rightarrow 0$ two point Schwinger function can be written as

$$
\widehat{G}_{\omega}^{2}(\mathbf{k})=(1+f(\lambda)) \frac{|\mathbf{k}|^{\eta_{z}}}{-i k_{0}+\omega k},
$$

with $f(\lambda)=O(\lambda)$ and independent from $\mathbf{k}$.

Remark. It is an easy consequence of our proof that the Schwinger functions do not depend on the parameter $\gamma$, but are only functions functions of $\lambda$ and $\mu$.

\subsection{WTi and chiral anomalies}

Once that the model is constructed and the OSa are verified, we can compute the WTi in the massless limit. We will show that

$$
\begin{gathered}
D_{\omega}(\mathbf{p}) \widehat{G}_{\omega, \omega^{\prime}}^{2,1, N, h}(\mathbf{p} ; \mathbf{k})= \\
=\delta_{\omega, \omega^{\prime}}\left[\widehat{G}_{\omega}^{2, N, h}(\mathbf{k}-\mathbf{p})-\widehat{G}_{\omega}^{2, N, h}(\mathbf{k})\right]+\widehat{\Delta}_{\omega, \omega^{\prime}}^{2,1, N}(\mathbf{p} ; \mathbf{k})
\end{gathered}
$$

where $\widehat{\Delta}_{\omega, \omega^{\prime}}^{2,1, N, h}(\mathbf{p} ; \mathbf{k})$ is a correction term which is formally vanishing if we replace $C_{h, N}^{-1}(\mathbf{k})$ by 1 .

The anomaly manifests itself in the fact that $\widehat{\Delta}_{\omega, \omega^{\prime}}^{2,1, N}$ is nonvanishing in the limit $N,-h \rightarrow \infty$; we will prove in fact the following Theorem. 


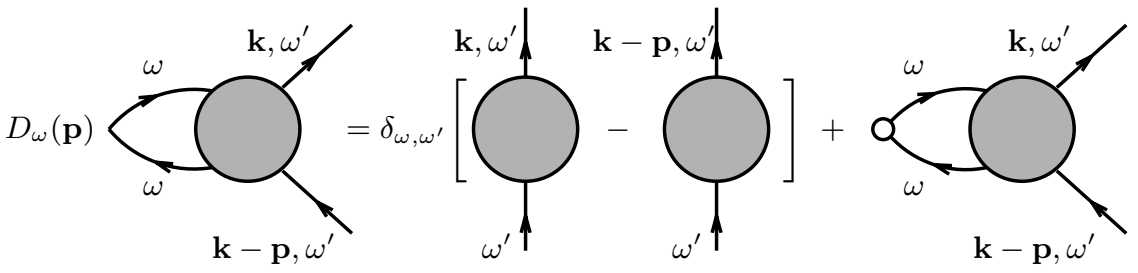

Figure 1: : Graphical representation of (26); the small circle in the last term represents the function in the r.h.s. of (111).

Theorem 2 Under the same conditions of Theorem 1, in the massless limit, i.e. $\mu=0$, it holds that for finite nonvanishing $\mathbf{k}, \mathbf{k}-\mathbf{p}, \mathbf{p}$

$$
\begin{gathered}
\widehat{\Delta}_{\omega, \omega^{\prime}}^{2,1, h}(\mathbf{p} ; \mathbf{k})=D_{\omega}(\mathbf{p}) \widehat{R}_{\omega, \omega^{\prime}}^{2,1, N, h}(\mathbf{p} ; \mathbf{k})+ \\
+\nu_{h, N}^{+} D_{\omega}(\mathbf{p}) \widehat{G}_{\omega, \omega^{\prime}}^{2,1, N, h}(\mathbf{p} ; \mathbf{k})+\nu_{-, h, N} D_{-\omega}(\mathbf{p}) \widehat{G}_{-\omega, \omega^{\prime}}^{2,1, N, h}(\mathbf{p} ; \mathbf{k}),
\end{gathered}
$$

where all the quantities appearing in this identity admit a $N,-h \rightarrow \infty$-limit, such that

$$
\nu_{-}=\frac{\lambda}{4 \pi}+O\left(\lambda^{2}\right), \quad \nu_{+}=c_{+} \lambda^{2}+O\left(\lambda^{2}\right)
$$

with $c_{+}<0,\left|\widehat{G}_{\omega, \omega^{\prime}}^{2,1}(\mathbf{p} ; \mathbf{k})\right|$ satisfies the bound (104) below, and

$$
\widehat{R}_{\omega, \omega^{\prime}}^{2,1}(\mathbf{p} ; \mathbf{k})=0 .
$$

It is immediate to check that the above result implies the WTi (6), with $a^{-1}=1-\nu^{-}-\nu^{+}$and $\bar{a}^{-1}=1+\nu^{-}-\nu^{+}$.

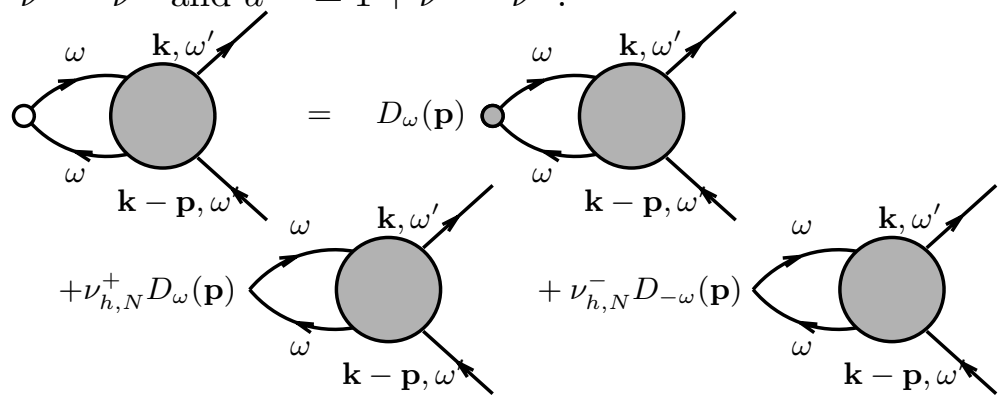

Figure 2: : Graphical representation of (27); the filled circle in the second term is the operator implicitly defined in $\S 4.1$

\subsection{Closed equation and additional anomaly}

It is easy to see (see for instance [BM4]) that the Schwinger functions of (13) in the massless limit verify the following SDe

$$
\widehat{G}_{\omega}^{2, N, h}(\mathbf{k})=\frac{\widehat{g}_{\omega}^{N, h}(\mathbf{k})}{Z_{N}}-\lambda \widehat{g}_{\omega}^{N, h}(\mathbf{k}) \int \frac{d \mathbf{p}}{(2 \pi)^{2}} \bar{\chi}_{N}(\mathbf{p}) \widehat{G}_{-\omega, \omega}^{2,1, N, h}(\mathbf{p} ; \mathbf{k}),
$$


where $\widehat{g}_{\omega}^{N, h}(\mathbf{k})=C_{h, N}^{-1}(\mathbf{k}) D_{\omega}(\mathbf{k})^{-1}$ and $\bar{\chi}_{N}(\mathbf{p})$ is a smooth function with support in $|\mathbf{p}| \leq 3 \gamma^{N+1}$, equal to 1 if $|\mathbf{p}| \leq 2 \gamma^{N+1}$ (we can insert it freely in the SDe, thanks to the support properties of the propagator).

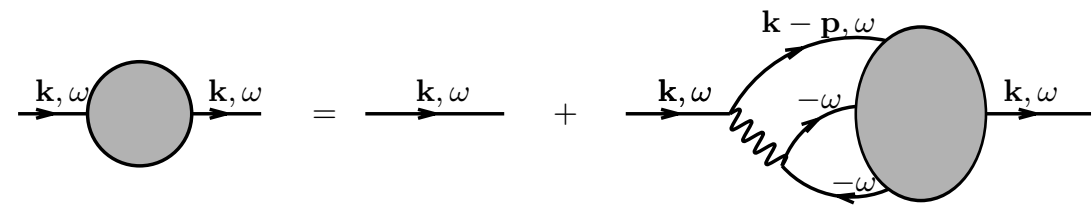

Figure 3: : Graphical representation of (30).

Inserting the WTi (26) in SDe (30) and using (27), we get

$$
\begin{aligned}
\widehat{G}_{\omega}^{2, N, h}(\mathbf{k}) & =\frac{\widehat{g}_{\omega}^{N, h}(\mathbf{k})}{Z_{N}}-\lambda \widehat{g}_{\omega}^{N, h}(\mathbf{k}) \int \frac{d \mathbf{p}}{(2 \pi)^{2}} \bar{\chi}_{h}(\mathbf{p}) \widehat{G}_{-\omega, \omega}^{2,1, h, N}(\mathbf{p} ; \mathbf{k})- \\
& -\lambda A_{+, h, N} \widehat{g}_{\omega}^{N, h}(\mathbf{k}) \int \frac{d \mathbf{p}}{(2 \pi)^{2}} \widetilde{\chi}(\mathbf{p}) \frac{\widehat{G}_{\omega}^{2, N, h}(\mathbf{k}-\mathbf{p})}{D_{-\omega}(\mathbf{p})}+ \\
& +\widehat{g}_{\omega}^{N, h}(\mathbf{k}) \sum_{\varepsilon} \lambda A_{\varepsilon, h, N} \int \frac{d \mathbf{p}}{(2 \pi)^{2}} \widetilde{\chi}(\mathbf{p}) \frac{D_{\varepsilon \omega}(\mathbf{p})}{D_{-\omega}(\mathbf{p})} \widehat{R}_{\varepsilon \omega, \omega}^{2,1, N, h}(\mathbf{p} ; \mathbf{k})
\end{aligned}
$$

where

$$
\begin{gathered}
A_{\varepsilon, h, N} \stackrel{\text { def }}{=} \quad \frac{a_{h, N}-\varepsilon \bar{a}_{h, N}}{2}, \\
a_{h, N}=\frac{1}{1-\nu_{h, N}^{-}-\nu_{h, N}^{+}} \quad, \quad \bar{a}_{h, N}=\frac{1}{1+\nu_{h, N}^{-}-\nu_{h, N}^{+}},
\end{gathered}
$$

$\bar{\chi}_{h}(\mathbf{p})$ is defined as $\bar{\chi}_{N}(\mathbf{p})$, with $h$ in place of $N$, and $\widetilde{\chi}(\mathbf{p})=\bar{\chi}_{N}(\mathbf{p})-\bar{\chi}_{h}(\mathbf{p})$ (so that the support of $\tilde{\chi}(\mathbf{p})$ is only for $2 \gamma^{h+1} \leq|\mathbf{p}| \leq 3 \gamma^{N+1}$ ). The bound (104) below implies that, if $\mathbf{k}$ is fixed to a non vanishing value, $\widehat{G}_{-\omega, \omega}^{2,1, h, N}(\mathbf{p} ; \mathbf{k})$ diverges more slowly that $|\mathbf{p}|^{-1 / 8}$, as $\mathbf{p} \rightarrow 0$; hence the second addend in the r.h.s. of (31) is vanishing in the limit $h=-\infty$.

If the last term in (31) were vanishing for $-h, N \rightarrow \infty$ (as the second addend), one would get a closed equation for $\widehat{G}_{\omega}^{2}$ which is identical to the one postulated in $[\mathrm{J}]$; it is just the formal Schwinger-Dyson equation combined with the WTi in the limit of removed cutoffs.

However this is not what happens; despite both WTi and SchwingerDyson equation are true in the limit, one cannot simply insert one in the other to obtain a closed equation. The last term is non vanishing and this is a additional anomaly effect which seems to be unnoticed in the literature.

Despite the presence of the additional anomaly, a closed equation (different with respect the one in $[\mathrm{J}]$ ) holds, as shown from the following theorem. 
Theorem 3 Under the same conditions of Theorem 1, in the massless limit there exist functions $\alpha_{\varepsilon, h, N}, \rho_{\varepsilon, h, N}$ such that, for non vanishing $\mathbf{k}$,

$$
\begin{gathered}
\widehat{g}_{\omega}^{N, h}(\mathbf{k}) \int \frac{d \mathbf{p}}{(2 \pi)^{2}} \widetilde{\chi}(\mathbf{p}) \frac{D_{\varepsilon \omega}(\mathbf{p})}{D_{-\omega}(\mathbf{p})} \widehat{R}_{\varepsilon \omega, \omega}^{2,1, N}(\mathbf{p} ; \mathbf{k})= \\
=-\alpha_{\varepsilon, h, N} \frac{\widehat{g}_{\omega}^{N, h}(\mathbf{k})}{Z_{N}}+\left(\alpha_{\varepsilon, h, N}+\rho_{\varepsilon, h, N}\right) \widehat{G}_{\omega}^{2, N, h}(\mathbf{k})+\widehat{R}_{\varepsilon}^{4, N, h}(\mathbf{k}),
\end{gathered}
$$

with

$$
\lim _{N,-h \rightarrow \infty} \widehat{R}_{\varepsilon}^{4, N, h}(\mathbf{k})=0
$$

and, in the limit of removed cutoff,

$$
\begin{array}{cl}
\alpha_{+}=c_{1} \lambda+O\left(\lambda^{2}\right), & \rho_{+}=c_{2} \lambda+O\left(\lambda^{2}\right), \\
\alpha_{-}=c_{3}+O(\lambda), & \rho_{-}=c_{4}+O(\lambda) .
\end{array}
$$

The above result says that, up to a vanishing term, the last addend in the r.h.s. of (31) can be written in terms of $g$ and $G^{2}$, so that a closed equation still holds in the limit, but different with respect to the one postulated in $[\mathrm{J}]$; in particular one gets a relation between the critical index $\eta_{z}$ and the anomalies $a, \bar{a}$, which is different with respect to the (3), that found in $[\mathrm{J}]$.

Corollary 1 The critical index of the massless two point Schwinger function (25) verifies

$$
\eta_{z}=\frac{\lambda}{2 \pi} \frac{a-\bar{a}}{1-\lambda \sum_{\varepsilon} A_{\varepsilon}\left(a_{\varepsilon}+\rho_{\varepsilon}\right)} .
$$

with $\sum_{\varepsilon} A_{\varepsilon}\left(a_{\varepsilon}+\rho_{\varepsilon}\right)=c_{0}+O\left(\lambda^{2}\right)$ with $c_{0}>0$.

\subsection{Lattice fermions and positive definiteness}

There are of course several ways to introduce a functional integral formulation of the Thirring model corresponding to different ways of regularizing the theory. The choice corresponding to (13) is the closest to the formal continuum limit (the regularized propagator is linear in $\mathbf{k}$ ) and this is convenient under many respects, for instance in the derivation of WTi and closed equation for the two point Schwinger function which we will discuss below. However such a choice has the big disadvantage that the crucial property of positive definiteness is quite difficult to prove; such a property is however automatically fulfilled with a lattice regularization. There is an extensive literature on the lattice fermions [MM]; if one simply replaces $k, k_{0}$ in the propagator with $a^{-1} \sin k a$ and $a^{-1} \sin k_{0} a$ the well known fermion doubling problem is encountered, namely that the massless fermion propagator has four poles instead of a single one. In the continuum limit $a \rightarrow 0$ this means that there are four fermion state per field component and such extra unwanted fermions influence possibly the physical behavior in a non trivial 
way. Several solutions have been proposed; we will follow here the Wilson formulation of adding a term to the free action, called Wilson term, to cancel the unwanted poles $[\mathrm{MM}]$. Then in the Wilson lattice regularization the fermionic integration is given by

$$
\begin{aligned}
& P_{Z_{a}}(d \psi) \stackrel{\text { def }}{=} \exp \left\{-\frac{Z_{a}}{L^{2}} \sum_{\omega, \omega^{\prime}= \pm} \sum_{\mathbf{k} \in \mathcal{D}_{a}}\left(\widehat{r}^{-1}(\mathbf{k})\right)_{\omega^{\prime}, \omega} \widehat{\psi}_{\mathbf{k}, \omega^{\prime}}^{+} \widehat{\psi}_{\mathbf{k}, \omega}^{-}\right\} . \\
& \cdot \prod_{\mathbf{k} \in \mathcal{D}_{a}} \prod_{\omega= \pm} \frac{\mathrm{d} \widehat{\psi}_{\mathbf{k}, \omega}^{+} \mathrm{d} \widehat{\psi}_{\mathbf{k}, \omega}^{-}}{\overline{\mathcal{N}}_{a}(\mathbf{k})}
\end{aligned}
$$

where the covariance $\widehat{r}_{\omega, \omega^{\prime}}(k)$ is defined as

$$
\widehat{r}_{\omega, \omega^{\prime}}(\mathbf{k}) \stackrel{\text { def }}{=} \frac{1}{e_{+}(\mathbf{k}) e_{-}(\mathbf{k})-\mu_{a}^{2}(\mathbf{k})}\left(\begin{array}{cc}
e_{-}(\mathbf{k}) & -\mu_{a}(\mathbf{k}) \\
-\mu_{a}(\mathbf{k}) & e_{+}(\mathbf{k})
\end{array}\right)_{\omega, \omega^{\prime}}
$$

with $k_{0}=\left(m_{0}+1 / 2\right) 2 \pi / L, k=(m+1 / 2) 2 \pi / L, n, n_{0}=-L / 2 a, 1, \ldots, L / 2 a-$ 1 ,

$$
\begin{aligned}
& e_{\omega}(\mathbf{k}) \stackrel{\text { def }}{=}-i \frac{\sin \left(k_{0} a\right)}{a}+\omega \frac{\sin (k a)}{a}, \\
& \mu_{a}(\mathbf{k}) \stackrel{\text { def }}{=} \mu+\frac{1-\cos \left(k_{0} a\right)}{a}+\frac{1-\cos (k a)}{a},
\end{aligned}
$$

and $\overline{\mathcal{N}}_{a}$ is the normalization. The generating functional is given by

$$
\begin{aligned}
& \int P_{Z_{a}}(d \psi) \exp \left\{-\lambda_{a} Z_{a}^{2} V(\psi)+\nu_{a} Z_{a} N(\psi)\right\} \\
& \cdot \exp \left\{Z_{a}^{(2)} \sum_{\omega} \int d \mathbf{x} J_{\mathbf{x}, \omega} \psi_{\mathbf{x}, \omega}^{+} \psi_{\mathbf{x}, \omega}^{-}+\sum_{\omega} \int d \mathbf{x}\left[\varphi_{\mathbf{x}, \omega}^{+} \psi_{\mathbf{x}, \omega}^{-}+\psi_{\mathbf{x}, \omega}^{+} \varphi_{\mathbf{x}, \omega}^{-}\right]\right\}
\end{aligned}
$$

where $N(\psi)=\sum_{\omega= \pm} \int d \mathbf{x} \psi_{\mathbf{x}, \omega}^{+} \psi_{\mathbf{x},-\omega}^{-}$. Note the presence of the term $(1-$ $\left.\cos \left(k_{0} a\right)\right) / a+(1-\cos (k a)) / a$ which has the effect that, in the massless case, only one pole is present. On the other hand it is not true, contrary to what happened in the previous case, that the massless case corresponds simply to $\mu=0$; the Wilson term breaks a parity symmetry leading to the generation though the interaction of a mass; we introduce then a counterterm $\nu_{a}$ to fix the mass proportional to $\mu$.

We call $S_{\underline{\sigma}, \underline{w}, \underline{\varepsilon}}^{N,(m ; n)}$ the Schwinger functions (17) (in the limit $a=0$ and $h=-\infty)$ and $\bar{S}_{\underline{\sigma} ; \underline{\underline{\omega}}, \underline{\underline{\varepsilon}}}^{a,(m ; n)}$ the Schwinger functions corresponding to (40); in $\S 5$ we shall prove the following theorem.

Theorem 4 Given $N>0$, let $a_{N}=\pi\left(4 \gamma^{N+1}\right)^{-1}$; if $\lambda$ is small enough, there exist functions $Z_{N}(\lambda), \mu g_{N}(\lambda)$ and $\lambda_{a_{N}}(\lambda), Z_{a_{N}}(\lambda), \nu_{a_{N}}(\lambda), \mu g_{a_{N}}(\lambda)$, such 
that, if all the points $\underline{\mathbf{z}}, \underline{\mathbf{x}}$ are different from each other, then $\bar{S}_{\underline{\sigma} ; \underline{\omega}, \underline{\underline{\varepsilon}}}^{\left.a_{N}, n\right)}(\underline{\mathbf{z}} ; \underline{\mathbf{x}})$ is well defined in the limit $N \rightarrow \infty$ and

$$
\lim _{N \rightarrow \infty}\left[S_{\underline{\sigma} ; \underline{\omega}, \underline{\varepsilon}}^{N,(m ; n)}(\underline{\mathbf{z}} ; \underline{\mathbf{x}})-\bar{S}_{\underline{\sigma} ; \underline{\omega}, \underline{\varepsilon}}^{a_{N},(m ; n)}(\underline{\mathbf{z}} ; \underline{\mathbf{x}})\right]=0 .
$$

The above result says that in the limit of removed cutoffs the two different regularizations of the Thirring model give the same Schwinger functions, if the "bare" parameters are suitably chosen.

The proof of the above results is based on many technical arguments, some of which were already proved in [BM1]-[BM4]; hence, in this paper we shall discuss in detail only the arguments not discussed in those papers.

\section{Continuum fermions with cutoff}

\subsection{Renormalization Group analysis}

The integration of the generating functional (13) is done almost exactly (essentially up to a trivial rescaling) as described in [BM1]-[BM4]; hence we briefly resume here such procedure to fix notations. It is possible to prove by induction that, for any $j: h \leq j \leq N$, there are a constant $E_{j}$, two positive functions $\tilde{Z}_{j}(\mathbf{k}), \tilde{\mu}_{j}(\mathbf{k})$ and two functionals $\mathcal{V}^{(j)}$ and $\mathcal{B}^{(j)}$, such that, if $Z_{j}=\max _{\mathbf{k}} \tilde{Z}_{j}(\mathbf{k})$,

$$
e^{\mathcal{W}(\varphi, J)}=e^{-L^{2} E_{j}} \int P_{\tilde{Z}_{j}, \tilde{\mu}_{j}, C_{h, j}}\left(d \psi^{[h, j]}\right) e^{-\mathcal{V}^{(j)}\left(\sqrt{Z_{j}} \psi^{[h, j]}\right)+\mathcal{B}^{(j)}\left(\sqrt{Z_{j}} \psi^{[h, j]}, \varphi, J\right)},
$$

where:

1. $P_{\widetilde{Z}_{j}, \tilde{\mu}_{j}, C_{h, j}}\left(d \psi^{[h, j]}\right)$ is the effective Grassmannian measure at scale $j$, equal to

$$
\begin{aligned}
& P_{\widetilde{Z}_{j}, \tilde{\mu}_{j}, C_{h, j}}\left(d \psi^{[h, j]}\right) \prod_{\mathbf{k}: C_{h, j}^{-1}(\mathbf{k})>0} \prod_{\omega, \omega^{\prime}= \pm 1} \frac{d \widehat{\psi}_{\mathbf{k}, \omega}^{[h, j]+} d \widehat{\psi}_{\mathbf{k}, \omega}^{[h, j]-}}{\mathcal{N}_{j}(\mathbf{k})} \cdot \\
& \cdot \exp \left\{-\frac{1}{L^{2}} \sum_{\mathbf{k}: C_{h, j}^{-1}(\mathbf{k})>0} C_{h, j}(\mathbf{k}) \tilde{Z}_{j}(\mathbf{k}) \sum_{\omega \pm 1} \widehat{\psi}_{\mathbf{k}, \omega}^{[h, j]+} T_{\omega, \omega^{\prime}}^{(j)}(\mathbf{k}) \widehat{\psi}_{\mathbf{k}, \omega^{\prime}}^{[h, j]-}\right\},
\end{aligned}
$$

with $T_{\omega, \omega^{\prime}}^{(j)}$ given by (15) with $\tilde{\mu}_{j}(\mathbf{k})$ replacing $\mu_{N}, C_{h, j}(\mathbf{k})^{-1}=\sum_{r=h}^{j} f_{r}(\mathbf{k})$ and $\mathcal{N}_{j}(\mathbf{k})$ a suitable normalization constant.

2. The effective potential on scale $j, \mathcal{V}^{(j)}(\psi)$, is a sum of monomial of Grassmannian variables multiplied by suitable kernels. i.e. it is of the form

$$
\mathcal{V}^{(j)}(\psi)=\sum_{n=1}^{\infty} \frac{1}{L^{4 n}} \sum_{\substack{\mathbf{k}_{1}, \ldots, \mathbf{k}_{2 n} \\ \omega_{1}, \ldots, \omega_{2 n}}}\left[\prod_{i=1}^{2 n} \widehat{\psi}_{\mathbf{k}_{i}, \omega_{i}}^{\sigma_{i}}\right] \widehat{W}_{2 n, \underline{\omega}}^{(j)}\left(\mathbf{k}_{1}, \ldots, \mathbf{k}_{2 n-1}\right) \delta\left(\sum_{i=1}^{2 n} \sigma_{i} \mathbf{k}_{i}\right),
$$


where $\sigma_{i}=+$ for $i=1, \ldots, n, \sigma_{i}=-$ for $i=n+1, \ldots, 2 n$ and $\underline{\omega}=\left(\omega_{1}, \ldots, \omega_{2 n}\right)$;

3. The effective source term at scale $j, \mathcal{B}^{(j)}\left(\sqrt{Z_{j}} \psi, \varphi, J\right)$, is a sum of monomials of Grassmannian variables and $\varphi^{ \pm}, J$ field, with at least one $\varphi^{ \pm}$ or one $J$ field; we shall write it in the form

$$
\mathcal{B}^{(j)}\left(\sqrt{Z_{j}} \psi, \varphi, J\right)=\mathcal{B}_{\varphi}^{(j)}\left(\sqrt{Z_{j}} \psi\right)+\mathcal{B}_{J}^{(j)}\left(\sqrt{Z_{j}} \psi\right)+W_{R}^{(j)}\left(\sqrt{Z_{j}} \psi, \varphi, J\right),
$$

where $\mathcal{B}_{\varphi}^{(j)}(\psi)$ and $\mathcal{B}_{J}^{(j)}(\psi)$ denote the sums over the terms containing only one $\varphi$ or $J$ field, respectively. $\mathcal{B}^{(j)}\left(\sqrt{Z_{j}} \psi, \varphi, J\right)$ can be written as sum over monomials of $\psi, \varphi, J$ multiplied by kernels $\widehat{W}_{2 n, n_{\varphi}, n_{J}, \underline{\omega}}^{(j)}$.

Of course (42) is true for $j=N$, with $\tilde{Z}_{N}(\mathbf{k})=Z_{N}, W_{R}^{(0)}=0$, and $\mathcal{V}^{(N)}(\psi), \mathcal{B}_{\varphi}^{(N)}, \mathcal{B}_{J}^{(N)}$ given implicitly by $(13)$. The kernels in $\widehat{W}^{(j)}, \mathcal{V}^{(j)}$ and $\mathcal{B}^{(j)}, j<N$, are functions of $\mu_{k}, Z_{k}$ and the effective couplings $\lambda_{k}$ (to be defined later), with $k \geq j$; the iterative construction below will inductively implies that the dependence on these variables is well defined.

We now begin to describe the iterative construction leading to (42). We introduce two operators $\mathcal{P}_{r}, r=0,1$, acting on the kernels $\widehat{W}^{(j)}$ in the following way

$$
\mathcal{P}_{0} \widehat{W}^{(j)}=\left.\widehat{W}^{(j)}\right|_{\tilde{\mu}_{j}, . . \mu_{N}=0}, \quad \mathcal{P}_{1} \widehat{W}^{(j)}=\left.\sum_{k \geq j, \mathbf{k}} \tilde{\mu}_{k}(\mathbf{k}) \frac{\partial \widehat{W}^{(j)}}{\partial \tilde{\mu}_{k}(\mathbf{k})}\right|_{\tilde{\mu}_{j}, . . \mu_{N}=0}
$$

We introduce also two operators $\mathcal{L}_{r}, r=0,1$, acting on the kernels $\widehat{W}^{(j)}$ in the following way:

1. If $n=1$,

$$
\begin{aligned}
& \mathcal{L}_{0} \widehat{W}_{2, \underline{\omega}}^{(j)}(\mathbf{k}) \stackrel{\text { def }}{=} \frac{1}{4} \sum_{\eta, \eta^{\prime}= \pm 1} \widehat{W}_{2, \underline{\omega}}^{(j)}\left(\overline{\mathbf{k}}_{\eta \eta^{\prime}}\right), \\
& \mathcal{L}_{1} \widehat{W}_{2, \underline{\omega}}^{(j)}(\mathbf{k}) \stackrel{\text { def }}{=} \frac{1}{4} \sum_{\eta, \eta^{\prime}= \pm 1} \widehat{W}_{2, \underline{\omega}}^{(j)}\left(\overline{\mathbf{k}}_{\eta \eta^{\prime}}\right)\left[\eta \frac{k_{0} L}{\pi}+\eta^{\prime} \frac{k L}{\pi}\right],
\end{aligned}
$$

where $\overline{\mathbf{k}}_{\eta \eta^{\prime}}=\left(\eta \frac{\pi}{L}, \eta^{\prime} \frac{\pi}{L}\right)$.

2. If $n=2, \mathcal{L}_{1} \widehat{W}_{4, \underline{\omega}} \stackrel{\text { def }}{=} 0$ and

$$
\mathcal{L}_{0} \widehat{W}_{4, \underline{\omega}}^{(j)}\left(\mathbf{k}_{1}, \mathbf{k}_{2}, \mathbf{k}_{3}\right) \stackrel{\operatorname{def}}{=} \widehat{W}_{4, \underline{\omega}}^{(j)}\left(\overline{\mathbf{k}}_{++}, \overline{\mathbf{k}}_{++}, \overline{\mathbf{k}}_{++}\right) .
$$

3. If $n>2, \mathcal{L}_{0} \widehat{W}_{2 n, \underline{\omega}}^{(j)} \stackrel{\text { def }}{=} \mathcal{L}_{1} \widehat{W}_{2 n, \underline{\omega}}^{(j)} \stackrel{\text { def }}{=} 0$. 
Given $\mathcal{L}_{j}, \mathcal{P}_{j}, j=0,1$ as above, we define the action of $\mathcal{L}$ on the kernels $\widehat{W}_{2 n, \underline{\omega}}^{(j)}$ as follows.

4. If $n=1$, then

$$
\mathcal{L} \widehat{W}_{2, \omega, \omega^{\prime}}^{(j)} \stackrel{\text { def }}{=}\left(\mathcal{L}_{0}+\mathcal{L}_{1}\right) \mathcal{P}_{0} \widehat{W}_{2, \omega, \omega^{\prime}}^{(j)}+\mathcal{L}_{0} \mathcal{P}_{1} \widehat{W}_{2, \omega, \omega^{\prime}}^{(j)} .
$$

5. If $n=2$, then $\mathcal{L} \widehat{W}_{4, \underline{\omega}}^{(j)} \stackrel{\text { def }}{=} \mathcal{L}_{0} \mathcal{P}_{0} \widehat{W}_{4, \underline{\omega}}^{(j)}$.

6. If $n>2$, then $\mathcal{L} \widehat{W}_{2 n, \underline{\omega}}^{(j)}=0$.

Note that $\mathcal{L}_{0} \mathcal{P}_{0} \widehat{W}_{2, \omega, \omega}^{(j)}=0$, because of the parity properties (in the exchange $\mathbf{k} \rightarrow-\mathbf{k}$ ) of the diagonal propagators, whose number is surely odd in each Feynmann graph contributing to $W_{2, \omega, \omega}^{(j)} ; \mathcal{L}_{0} \mathcal{P}_{1} \widehat{W}_{2, \omega, \omega}^{(j)}=0$, because there are no contributions of first order in $\mu_{k} ; \mathcal{P}_{0} \widehat{W}_{2, \omega,-\omega}^{(j)}=0$, since the only way to get a contribution to $\widehat{W}_{2, \omega,-\omega}^{(j)}$ is to use at least one antidiagonal propagator. Therefore (49) reads

$$
\mathcal{L} \widehat{W}_{2, \omega, \omega}^{(j)}=\mathcal{L}_{1} \mathcal{P}_{0} \widehat{W}_{2, \omega, \omega}^{(j)}, \quad \mathcal{L} \widehat{W}_{2, \omega,-\omega}^{(j)}=\mathcal{L}_{0} \mathcal{P}_{1} \widehat{W}_{2, \omega,-\omega}^{(j)} .
$$

Note also that $\mathcal{L}^{2} \mathcal{V}^{(j)}=\mathcal{L} \mathcal{V}^{(j)}$. The effect of $\mathcal{L}$ on $\mathcal{V}^{(j)}$ is, by definition, to replace on the r.h.s. of (44) $\widehat{W}_{2 n, \underline{\omega}}^{(j)}$ with $\mathcal{L} \widehat{W}_{2 n, \underline{\omega}}^{(j)}$; we get

$$
\mathcal{L} \mathcal{V}^{(j)}\left(\psi^{[h, j]}\right)=z_{j} F_{\zeta}^{[h, j]}+s_{j} F_{\sigma}^{[h, j]}+l_{j} F_{\lambda}^{[h, j]},
$$

where $z_{j}, a_{j}$ and $l_{j}$ are real numbers and

$$
\begin{aligned}
& F_{\zeta}^{[h, j]}=\frac{1}{L^{2}} \sum_{\omega} \sum_{\mathbf{k}: C_{h, j}^{-1}(\mathbf{k})>0} D_{\omega}(\mathbf{k}) \widehat{\psi}_{\mathbf{k}, \omega}^{[h, j]+} \widehat{\psi}_{\mathbf{k}, \omega}^{[h, j]-} \\
& F_{\sigma}^{[h, j]}=\frac{1}{L^{2}} \sum_{\omega} \sum_{\mathbf{k}: C_{h, j}^{-1}(\mathbf{k})>0} \widehat{\psi}_{\mathbf{k}, \omega}^{[h, j]+} \widehat{\psi}_{\mathbf{k},-\omega}^{[h, j]-} \\
& F_{\lambda}^{[h, j]}=\frac{1}{L^{8}} \sum_{\mathbf{k}_{1}, \ldots, \mathbf{k}_{4}: C_{h, j}^{-1}\left(\mathbf{k}_{i}\right)>0} \widehat{\psi}_{\mathbf{k}_{1},+}^{[h, j]+} \widehat{\psi}_{\mathbf{k}_{2},+}^{[h, j]-} \widehat{\psi}_{\mathbf{k}_{3},-}^{[h, j]+} \widehat{\psi}_{\mathbf{k}_{4},-}^{[h, j]-} \delta\left(\mathbf{k}_{1}-\mathbf{k}_{2}+\mathbf{k}_{3}-\mathbf{k}_{4}\right) .
\end{aligned}
$$

Analogously, we write $\mathcal{B}^{(j)}=\mathcal{L B}^{(j)}+\mathcal{R} \mathcal{B}^{(j)}, \mathcal{R}=1-\mathcal{L}$, according to the following definition. First of all, we put $\mathcal{L} W_{R}^{(j)}=0$. Let us consider now $\mathcal{B}_{J}^{(j)}\left(\sqrt{Z_{j}} \psi\right)$. It is easy to see that the field $J$ is equivalent, from the point of view of dimensional considerations, to two $\psi$ fields. Hence, the only terms which need to be renormalized are those of second order in $\psi$, which are indeed marginal. We shall use for them the definition

$$
\mathcal{B}_{J}^{(j, 2)}\left(\sqrt{Z_{j}} \psi\right)=\sum_{\omega, \tilde{\omega}} \frac{1}{L^{4}} \sum_{\mathbf{p}, \mathbf{k}} \widehat{B}_{\omega, \tilde{\omega}}^{(j)}(\mathbf{p}, \mathbf{k}) \widehat{J}_{\mathbf{p}, \omega}\left(\sqrt{Z_{j}} \widehat{\psi}_{\mathbf{p}+\mathbf{k}, \tilde{\omega}}^{[h, j]+}\right)\left(\sqrt{Z_{j}} \widehat{\psi}_{\mathbf{k}, \tilde{\omega}}^{[h, j]-}\right) .
$$


We regularize $\mathcal{B}_{J}^{(j, 2)}\left(\sqrt{Z_{j}} \psi\right)$, in analogy to what we did for the effective potential, by decomposing it as the sum of $\mathcal{L B}_{J}^{(j, 2)}\left(\sqrt{Z_{j}} \psi\right)$ and $\mathcal{R} \mathcal{B}_{J}^{(j, 2)}\left(\sqrt{Z_{j}} \psi\right)$, where $\mathcal{L}$ is defined through its action on $\widehat{B}_{\omega, \tilde{\omega}}^{(j)}(\mathbf{p}, \mathbf{k})$ in the following way:

$$
\mathcal{L} \widehat{B}_{\omega, \tilde{\omega}}^{(j)}(\mathbf{p}, \mathbf{k})=\frac{1}{4} \delta_{\omega, \tilde{\omega}} \sum_{\eta, \eta^{\prime}= \pm 1} \mathcal{P}_{0} \widehat{B}_{\omega, \tilde{\omega}}^{(j)}\left(0, \overline{\mathbf{k}}_{\eta, \eta^{\prime}}\right)
$$

note that $\mathcal{L} \widehat{B}_{\omega,-\omega}^{(j)}=0$ because of the symmetry property

$$
\widehat{g}_{\omega}^{(j)}(\mathbf{k})=-i \omega \widehat{g}_{\omega}^{(j)}\left(\mathbf{k}^{*}\right) \quad, \quad \mathbf{k}=\left(k, k_{0}\right), \quad \mathbf{k}^{*}=\left(-k_{0}, k\right) .
$$

We get

$$
\mathcal{L B}_{J}^{(j, 2)}\left(\sqrt{Z_{j}} \psi\right)=\sum_{\omega} \frac{Z_{j}^{(2)}}{Z_{j}} \int \mathrm{d} \mathbf{x} J_{\mathbf{x}, \omega}\left(\sqrt{Z_{j}} \psi_{\mathbf{x}, \omega}^{+}\right)\left(\sqrt{Z_{j}} \psi_{\mathbf{x}, \omega}^{-}\right),
$$

which defines the renormalization constant $Z_{j}^{(2)}$; we shall extend this definition to $j=N$ by putting, in agreement with (13), $Z_{N}^{(2)}=Z_{N}$.

Finally we have to define $\mathcal{L}$ for $\mathcal{B}_{\varphi}^{(j)}\left(\sqrt{Z_{j}} \psi\right)$; we want to show that, by a suitable choice of the localization procedure, if $j \leq N-1$, it can be written in the form

$$
\begin{gathered}
\mathcal{B}_{\varphi}^{(j)}\left(\sqrt{Z_{j}} \psi\right)=\sum_{\omega, \omega^{\prime}} \sum_{i=j+1}^{N} \int d \mathbf{x} d \mathbf{y} \cdot \\
\cdot\left[\varphi_{\mathbf{x}, \omega}^{+} g_{\omega, \omega^{\prime}}^{Q,(i)}(\mathbf{x}-\mathbf{y}) \frac{\partial}{\partial \psi_{\mathbf{y}, \omega^{\prime}}^{+}} \mathcal{V}^{(j)}\left(\sqrt{Z_{j}} \psi\right)+\frac{\partial}{\partial \psi_{\mathbf{y}, \omega}^{-}} \mathcal{V}^{(j)}\left(\sqrt{Z_{j}} \psi\right) g_{\omega, \omega^{\prime}}^{Q,(i)}(\mathbf{y}-\mathbf{x}) \varphi_{\mathbf{x}, \omega^{\prime}}^{-}\right]+ \\
+\sum_{\omega, \omega^{\prime}} \frac{1}{L^{2}} \sum_{\mathbf{k}: C_{h, j}^{-1}(\mathbf{k})>0}\left[\widehat{\psi}_{\mathbf{k}, \omega}^{[h, j]+} \widehat{Q}_{\omega, \omega^{\prime}}^{(j+1)}(\mathbf{k}) \widehat{\varphi}_{\mathbf{k}, \omega^{\prime}}^{-}+\widehat{\varphi}_{\mathbf{k}, \omega}^{+} \widehat{Q}_{\omega, \omega^{\prime}}^{(j+1)}(\mathbf{k}) \widehat{\psi}_{\mathbf{k}, \omega^{\prime}}^{[h, j]-}\right]
\end{gathered}
$$

where $\widehat{g}_{\omega, \omega^{\prime}}^{Q,(i)}(\mathbf{k})=\sum_{\omega^{\prime \prime}} \widehat{g}_{\omega, \omega^{\prime \prime}}^{(i)}(\mathbf{k}) \widehat{Q}_{\omega^{\prime \prime}, \omega^{\prime}}^{(i)}(\mathbf{k}), g_{\omega, \omega^{\prime \prime}}^{(i)}$ is the renormalized propagator of the field on scale $j$ (see (63) below for a precise definition) and $\widehat{Q}_{\omega, \omega^{\prime}}^{(j)}(\mathbf{k})$ is defined inductively by the relations

$$
\begin{aligned}
& \widehat{Q}_{\omega, \omega^{\prime}}^{(j)}(\mathbf{k})=\widehat{Q}_{\omega, \omega^{\prime}}^{(j+1)}(\mathbf{k})-z_{j} Z_{j} D_{\omega}(\mathbf{k}) \sum_{i=j+1}^{N} \widehat{g}_{\omega, \omega^{\prime}}^{Q,(i)}(\mathbf{k})-s_{j} Z_{j} \sum_{i=j+1}^{N} \widehat{g}_{\omega,-\omega^{\prime}}^{Q,(i)}(\mathbf{k}), \\
& \widehat{Q}_{\omega, \omega^{\prime}}^{(0)}(\mathbf{k})=1 .
\end{aligned}
$$

The $\mathcal{L}$ operation for $\mathcal{B}_{\varphi}^{(j)}$ is defined by decomposing $\mathcal{V}^{(j)}$ in the r.h.s. of (57) as $\mathcal{L} \mathcal{V}^{(j)}+\mathcal{R} \mathcal{V}^{(j)}, \mathcal{L} \mathcal{V}^{(j)}$ being defined by $(51)$. 
After writing $\mathcal{V}^{(j)}=\mathcal{L} \mathcal{V}^{(j)}+\mathcal{R} \mathcal{V}^{(j)}$ and $\mathcal{B}^{(j)}=\mathcal{L} \mathcal{B}^{(j)}+\mathcal{R} \mathcal{B}^{(j)}$, the next step is to renormalize the free measure $P_{\tilde{Z}_{j}, \tilde{\mu}_{j}, C_{h, j}}\left(d \psi^{[h, j]}\right)$, by adding to it part of the r.h.s. of (51). We get that (42) can be written as

$$
e^{-L^{2} t_{j}} \int P_{\tilde{Z}_{j-1}, \tilde{\mu}_{j-1}, C_{h, j}}\left(d \psi^{[h, j]}\right) e^{-\tilde{\mathcal{V}}^{(j)}\left(\sqrt{Z_{j}} \psi^{[h, j]}\right)+\tilde{\mathcal{B}}^{(j)}\left(\sqrt{Z_{j}} \psi^{[h, j]}\right)},
$$

where, since $\tilde{Z}_{j}(\mathbf{k})=Z_{j} \equiv \max _{\mathbf{k}} \tilde{Z}_{j}(\mathbf{k})$ and $\tilde{\mu}_{j}(\mathbf{k})=\mu_{j} \equiv\left(Z_{j+1} / Z_{j}\right)\left(\mu_{j+1}+\right.$ $\left.s_{j+1}\right)$, if $C_{h, j}^{-1}(\mathbf{k}) \neq 0$, then

$$
\begin{gathered}
\tilde{Z}_{j-1}(\mathbf{k})=Z_{j}\left[1+C_{h, j}^{-1}(\mathbf{k}) z_{j}\right], \quad \tilde{\mu}_{j-1}(\mathbf{k})=\frac{Z_{j}}{\tilde{Z}_{j-1}(\mathbf{k})}\left[\mu_{j}+C_{h, j}^{-1}(\mathbf{k}) s_{j}\right] \\
\tilde{\mathcal{V}}^{(j)}\left(\psi^{[h, j]}\right)=\mathcal{V}^{(j)}\left(\psi^{[h, j]}\right)-z_{j} F_{\zeta}^{[h, j]}-s_{j} F_{\sigma}^{[h, j]}
\end{gathered}
$$

and the factor $\exp \left(-L^{2} t_{j}\right)$ in (59) takes into account the different normalization of the two measures. Moreover

$$
\tilde{\mathcal{B}}^{(j)}\left(\sqrt{Z_{j}} \psi^{[h, j]}\right)=\tilde{\mathcal{B}}_{\varphi}^{(j)}\left(\sqrt{Z_{j}} \psi^{[h, j]}\right)+\mathcal{B}_{J}^{(j)}\left(\sqrt{Z_{j}} \psi^{[h, j]}\right)+W_{R}^{(j)},
$$

where $\tilde{\mathcal{B}}_{\varphi}^{(j)}$ is obtained from $\mathcal{B}_{\varphi}^{(j)}$ by inserting (62) in the second line of (57) and by absorbing the terms proportional to $z_{j}, s_{j}$ in the terms in the third line of (57).

If $j>h$, the r.h.s of (59) can be written as

$$
\begin{aligned}
& e^{-L^{2} t_{j}} \int P_{\tilde{Z}_{j-1}, \mu_{j-1}, C_{h, j-1}}\left(d \psi^{[h, j-1]}\right) \int P_{Z_{j-1}, \mu_{j-1}, \tilde{f}_{j}^{-1}}\left(d \psi^{(j)}\right) \cdot \\
& \cdot e^{-l_{j} F_{\lambda}\left(\sqrt{Z_{j}} \psi^{[h, j]}\right)-\mathcal{R} \mathcal{V}\left(\sqrt{Z_{j}} \psi^{[h, j]}\right)+\tilde{\mathcal{B}}^{(j)}}\left(\sqrt{Z_{j}} \psi^{[h, j]}\right)
\end{aligned}
$$

where $\tilde{f}_{j}(\mathbf{k})=f_{j}(\mathbf{k}) Z_{j-1}\left[\tilde{Z}_{j-1}(\mathbf{k})\right]^{-1}$.

The above integration procedure is done till the scale $h^{*}=\max \left\{h, \bar{h}^{*}\right\}$, where $\bar{h}^{*}$ is the maximal $j$ such that $\gamma^{j} \leq \mu_{j}$. If $\bar{h}^{*}<j \leq N$, by using the Gevray property (11) of $\chi_{0}$, see $[\mathrm{DR}]$, we get

$$
\begin{aligned}
\left|g_{\omega, \omega}^{(j)}(\mathbf{x}, \mathbf{y})\right| & \leq \frac{C}{Z_{j-1}} \gamma^{j} e^{-c \sqrt{\gamma^{j}|\mathbf{x}-\mathbf{y}|}} \\
\left|g_{\omega,-\omega}^{(j)}(\mathbf{x}, \mathbf{y})\right| & \leq \frac{C}{Z_{j-1}}\left(\frac{\mu_{j}}{\gamma^{j}}\right) \gamma^{j} e^{-c \sqrt{\gamma^{j}|\mathbf{x}-\mathbf{y}|}},
\end{aligned}
$$

where $C$ and $c$ are suitable constants; moreover,

$$
\begin{aligned}
& \left|g_{\omega, \omega}^{\left(\leq \bar{h}^{*}\right)}(\mathbf{x}, \mathbf{y})\right| \leq \frac{C}{Z_{\bar{h}^{*}-1}} \gamma^{\bar{h}^{*}} e^{-c \sqrt{\gamma^{\bar{h}^{*}}|\mathbf{x}-\mathbf{y}|}}, \\
& \left|g_{\omega,-\omega}^{\left(\leq \bar{h}^{*}\right)}(\mathbf{x}, \mathbf{y})\right| \leq \frac{C}{Z_{\bar{h}^{*}-1}}\left(\frac{\mu_{\bar{h}^{*}}}{\gamma^{\bar{h}^{*}}}\right) \gamma^{\bar{h}^{*}} e^{-c \sqrt{\gamma^{\bar{h}^{*}}|\mathbf{x}-\mathbf{y}|}} .
\end{aligned}
$$


Note that the propagator $\widehat{g}_{\omega}^{Q,(i)}(\mathbf{k})$ is equivalent to $\widehat{g}_{\omega}^{(i)}(\mathbf{k})$, as concerns the dimensional bounds, since the sum in the r.h.s. of (58) contains at most two nonvanishing terms. We now rescale the field so that

$$
\begin{aligned}
& l_{j} F_{\lambda}\left(\sqrt{Z_{j}} \psi^{[h, j]}\right)+\mathcal{R} \mathcal{V}\left(\sqrt{Z_{j}} \psi^{[h, j]}\right)=\widehat{\mathcal{V}}^{(j)}\left(\sqrt{Z_{j-1}} \psi^{[h, j]}\right), \\
& \tilde{\mathcal{B}}^{(j)}\left(\sqrt{Z_{j}} \psi^{[h, j]}\right)=\widehat{\mathcal{B}}^{(j)}\left(\sqrt{Z_{j-1}} \psi^{[h, j]}\right) ;
\end{aligned}
$$

it follows that $\mathcal{L} \widehat{\mathcal{V}}^{(j)}\left(\psi^{[h, j]}\right)=\lambda_{j} F_{\lambda}^{[h, j]}$ where $\lambda_{j}=\left(Z_{j} Z_{j-1}^{-1}\right)^{2} l_{j}$; we shall extend this definition to $j=N$ by putting, in agreement with (13), $\lambda_{N}=\lambda$. If we now define

$$
\begin{aligned}
& e^{-\mathcal{V}^{(j-1)}\left(\sqrt{Z_{j-1}} \psi^{[h, j-1]}\right)+\mathcal{B}^{(j-1)}\left(\sqrt{Z_{j-1}} \psi^{[h, j-1]}\right)-L^{2} E_{j}}=
\end{aligned}
$$

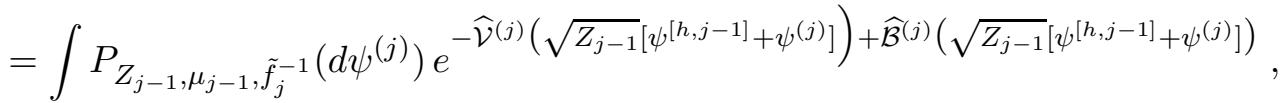

it is easy to see that $\mathcal{V}^{(j-1)}$ and $\mathcal{B}^{(j-1)}$ are of the same form of $\mathcal{V}^{(j)}$ and $\mathcal{B}^{(j)}$ and that the procedure can be iterated. Note that the above procedure allows, in particular, to write $\lambda_{j}, Z_{j}, \mu_{j}$, for any $j$ such that $N>j \geq h^{*}$, in terms of $\lambda_{j^{\prime}}, Z_{j^{\prime}}, \mu_{j^{\prime}}, j^{\prime}>j$.

At the end of the iterative integration procedure, we get

$$
\mathcal{W}(\varphi, J)=-L^{2} E_{L}+\sum_{m^{\varphi}+n^{J} \geq 1} S_{2 m^{\varphi}, n^{J}}^{(h)}(\varphi, J)
$$

where $E_{L}$ is the free energy and $S_{2 m^{\varphi}, n^{J}}^{(h)}(\varphi, J)$ are suitable functionals, which can be expanded, as well as $E_{L}$, the effective potentials and the various terms in the r.h.s. of (45) and (44), in terms of trees. We do not repeat here the analysis leading to the tree expansion, as it is essentially identical to the one for instance in $\S 3$ of [BM1], and we quote the results; it turns out the kernels $S_{2 m^{\varphi}, n^{J}}^{(h)}(\varphi, J)$ can be written as in formula (102) of [BM2]:

$$
\begin{gathered}
S_{2 m^{\varphi}, n^{J}}^{(h)}(\varphi, J)=\sum_{n=0}^{\infty} \sum_{j_{0}=h^{*}-1}^{N-1} \sum_{\underline{\omega}} \sum_{\tau \in \mathcal{T}_{j_{0}, n, 2 m}, n^{J}} \sum_{\substack{\mathbf{P} \in \mathcal{P} \\
\left|P_{v_{0}}\right|=2 m \varphi}} \\
\int d \underline{\mathbf{x}} \prod_{i=1}^{2 m^{\varphi}} \varphi_{\mathbf{x}_{i}, \omega_{i}}^{\sigma_{i}} \prod_{r=1}^{n^{J}} J_{\mathbf{x}_{2 m^{\varphi}+r}, \omega_{2 m} \varphi+r} S_{2 m^{\varphi}, n^{J}, \tau, \underline{\omega}}(\underline{\mathbf{x}}),
\end{gathered}
$$

where we refer to $\S 3.4$ of [BM2] for the notation. In particular,

- $\mathcal{T}_{j_{0}, n, 2 m^{\varphi}, n^{J}}$ is a family of trees (identical to the those defined in $\S 3.2$ of [BM2], up to the (trivial) difference that the maximum scale of the vertices is $N+1$ instead of +1 ), with root at scale $j_{0}, n$ normal endpoints (i.e. endpoints not associated to $\varphi$ or $J$ fields), $n^{\varphi}=2 m^{\varphi}$ endpoints of type $\varphi$ and $n^{J}$ endpoints of type $J$. 
- If $v$ is a vertex of the tree $\tau, P_{v}$ is a set of labels which distinguish the external fields of $v$, that is the field variables of type $\psi$ which belong to one of the endpoints following $v$ and either are not yet contracted in the vertex $v$ (we shall call $P_{v}^{(n)}$ the set of these variables) or are contracted with the $\psi$ variable of an endpoint of type $\varphi$ through a propagator $g^{Q\left(h_{v}\right)}$; note that $\left|P_{v}\right|=\left|P_{v}^{(n)}\right|+n_{v}^{\varphi}$, if $n_{v}^{\varphi}$ is the number of endpoints of type $\varphi$ following $v$.

$-\mathbf{x}_{v}$, if $v$ is not an endpoint, is the family of all space-time points associated with one of the endpoints following $v$.

\subsection{Convergence of the RG expansion}

In order to control the RG expansion, it is sufficient to show that $\bar{\lambda}_{h} \equiv$ $\max _{h \leq j \leq N}\left|\lambda_{j}\right|$ stays small if $\lambda=\lambda_{N}$ is small enough. This property is surely true if $|h-N|$ is at most of order $\lambda^{-1}$, but to prove that it is true for any $h, N$ is quite nontrivial. In $\S 4.3$, by using WTi and SDe, we shall prove the following Theorem, essentially taken from [BM4].

Theorem 5 There exists a constant $\varepsilon_{1}$, independent of $N$, such that, if $|\lambda| \leq \varepsilon_{1}$, the constants $\lambda_{j}, Z_{j}, Z_{j}^{(2)}$ and $\mu_{j}$ are well defined for any $j \leq N$; moreover there exist suitable sequences $\widehat{\lambda}_{j}, \widehat{Z}_{j}, \widehat{Z}_{j}^{(2)}$ and $\widehat{\mu}_{j}$, defined for $j \leq 0$ and independent of $N$, such that $\lambda_{j}=\widehat{\lambda}_{j-N}, Z_{j}=\widehat{Z}_{j-N}, Z_{j}^{(2)}=\widehat{Z}_{j-N}^{(2)}$ and $\mu_{j}=\widehat{\mu}_{j-N}$. The sequence $\widehat{\lambda}_{j}$ converges, as $j \rightarrow-\infty$, to a function $\lambda_{-\infty}(\lambda)=\lambda+O\left(\lambda^{2}\right)$, such that

$$
\left|\widehat{\lambda}_{j}-\lambda_{-\infty}\right| \leq C \lambda^{2} \gamma^{j / 4}
$$

Finally, there exist $\eta_{\mu}=-a_{\mu} \lambda+O\left(\lambda^{2}\right)$ and $\eta_{z}=a_{z} \lambda^{2}+O\left(\lambda^{3}\right)$, with $a_{\mu}$ and $a_{z}$ strictly positive, such that, for any $j \leq 0,\left|\log _{\gamma}\left(\widehat{Z}_{j-1} / \widehat{Z}_{j}\right)-\eta_{z}\right| \leq C \lambda^{2} \gamma^{j / 4}$, $\left|\log _{\gamma}\left(\widehat{Z}_{j-1}^{(2)} / \widehat{Z}_{j}^{(2)}\right)-\eta_{z}\right| \leq C \lambda^{2} \gamma^{j / 4}$ and $\left|\log _{\gamma}\left(\widehat{\mu}_{j-1} / \widehat{\mu}_{j}\right)-\eta_{\mu}\right| \leq C|\lambda| \gamma^{j / 4}$.

Remark. Note that the definitions of $\lambda_{j}, \mu_{j}, Z_{j}$ and $Z_{j}^{(2)}$ are independent of the $\mu$ value; however, in the theory with $\mu \neq 0$, there appear only their values with $j \geq \bar{h}^{*}$.

The above result implies that we can remove the cutoffs and take the limit $N,-h \rightarrow \infty$, by choosing the normalization conditions

$$
Z_{0}=1, \quad \mu_{0}=\mu
$$

In fact, by using (71), it is easy to prove that, if $Z_{N}=Z_{N}^{(2)}=\left[\prod_{i=1}^{N}\left(Z_{j-1} / Z_{j}\right)\right]^{-1}$ and $\mu_{N}=\left[\prod_{i=1}^{N}\left(\mu_{j-1} / \mu_{j}\right)\right]^{-1}$, then

$$
\mu_{j}=\mu \gamma^{-\eta_{\mu} j} F_{1, j, N}(\lambda), \quad Z_{j}=\gamma^{-\eta_{z} j} F_{2, j, N}(\lambda), \quad Z_{j}^{(2)}=\zeta(\lambda) \gamma^{-\eta_{z} j} F_{3, j, N}(\lambda),
$$


where

$$
\zeta(\lambda)=\prod_{j=-\infty}^{0} \frac{\widehat{Z}_{j-1}^{(2)} \widehat{Z}_{j}}{\widehat{Z}_{j}^{(2)} \widehat{Z}_{j-1}}
$$

and $F_{i, j, N}(\lambda), i=1,2,3$, satisfy the conditions

$$
F_{i, 0, N}(\lambda)=1, \quad\left|F_{i, j, N}(\lambda)-1\right| \leq C|\lambda|^{2} \gamma^{-[N-\max \{j, 0\}] / 4} .
$$

Note also that the first of (72) implies that, in the limit $N,-h \rightarrow \infty$, if $[x]$ denotes the largest integer $\leq x$,

$$
\bar{h}^{*}=\left[\frac{\log _{\gamma}|\mu|}{1-\eta_{\mu}}\right] .
$$

Moreover, the proof of Theorem 5 implies that the critical indices $\eta_{z}$ and $\eta_{\mu}$ are given by tree expansions, such that everywhere the constants $\lambda_{j}$ and $Z_{j}$ are substituted with $\lambda_{-\infty}$ and $\gamma^{-\eta_{z} j}$. In particular $\eta_{z}$ is the solution of an equation of the form

$$
\eta_{z}=a_{z} \lambda_{-\infty}^{2}+\lambda_{-\infty}^{4} H\left(\lambda_{-\infty}, \eta_{z}\right),
$$

which allows to explicitly calculate the perturbative expansion of $\eta_{z}$ through an iteratively procedure.

Remark. The normalization conditions (71) could also include the value of $Z_{j}^{(2)}$ for $j=0$, but we have chosen to fix the value of $Z_{j}^{(2)}$ for $j=N$, by putting it equal to $Z_{N}$. A different choice would only change the value of $\zeta(\lambda)$ by an arbitrary finite constant.

\subsection{The Schwinger functions}

Theorem 5 allows us to control the expansion of the Schwinger functions, by using the following bound for the kernels appearing in the expansion (69):

$$
\begin{aligned}
& \int d \underline{\mathbf{x}}\left|S_{2 m^{\varphi}, n^{J}, \tau, \underline{\omega}}(\underline{\mathbf{x}})\right| \leq L^{2} C^{2 m_{\varphi}+n_{J}}\left(C \bar{\lambda}_{j_{0}}\right)^{n} \gamma^{-j_{0}\left(-2+m^{\varphi}+n^{J}\right)} . \\
& \cdot \prod_{i=1}^{2 m^{\varphi}} \frac{\gamma^{-h_{i}}}{\left(Z_{h_{i}}\right)^{1 / 2}} \prod_{r=1}^{n^{J}} \frac{Z_{\bar{h}_{r}}^{(2)}}{Z_{\bar{h}_{r}}} \prod_{\text {v not e.p. }}\left(\frac{Z_{h_{v}}}{Z_{h_{v}-1}}\right)^{\left|P_{v}\right| / 2} \gamma^{-d_{v}},
\end{aligned}
$$

where $h_{i}$ is the scale of the propagator linking the $i$-th endpoint of type $\varphi$ to the tree, $\bar{h}_{r}$ is the scale of the $r$-th endpoint of type $J$ and

$$
d_{v}=-2+\left|P_{v}\right| / 2+n_{v}^{J}+\tilde{z}\left(P_{v}\right),
$$


with

$$
\tilde{z}\left(P_{v}\right)= \begin{cases}3 / 4 & \text { if }\left|P_{v}\right|=4, n_{v}^{\varphi}=0,1, n_{v}^{J}=0, \\ 3 / 2 & \text { if }\left|P_{v}\right|=2, n_{v}^{\varphi}=0,1, n_{v}^{J}=0, \\ 3 / 4 & \text { if }\left|P_{v}\right|=2, n_{v}^{\varphi}=0, n_{v}^{J}=1, \\ 0 & \text { otherwise. }\end{cases}
$$

The above bound has a simple dimensional interpretation; how to prove it rigorously has been explained in detail in the very similar model studied in [BM1] (see also $\S 3$ of [BM2]). We simply remark here that, had we defined $\mathcal{L}=0$, we would have obtained a bound similar to $(77)$ with $\tilde{z}\left(P_{v}\right)=0$ in (79). The regularization procedure has the effect that the vertex dimension $d_{v}$ gets an extra $\tilde{z}\left(P_{v}\right)$, whose value can be understood in the following way. If we apply the regularizing operator $1-\mathcal{L}_{0}$ to the kernel associated with the vertex $v$, the bound improves by a dimensional factor $\gamma^{h_{v^{\prime}}-h_{v}}$, if $v^{\prime}$ is the first non trivial vertex preceding $v$; if we apply $1-\mathcal{L}_{0}-\mathcal{L}_{1}$, the bound improves by a factor $\gamma^{2\left(h_{v^{\prime}}-h_{v}\right)}$. Moreover, if to a kernel associated with the vertex $v$ the operator $1-\mathcal{P}_{0}$ is applied, the bound improves by a factor

$$
\begin{aligned}
& \left|\mu_{h_{v}}\right| \gamma^{-h_{v}} \leq\left|\mu_{h^{*}}\right|\left|\mu_{h_{v}} / \mu_{h^{*}}\right| \gamma^{-h_{v}} \leq \gamma^{h^{*}} \gamma^{c \bar{\lambda}_{j_{0}}\left(h_{v}-h^{*}\right)} \gamma^{-h_{v}}= \\
& =\gamma^{\left(1-c \bar{\lambda}_{j_{0}}\right)\left(h^{*}-h_{v}\right)} \leq \gamma^{\frac{3}{4}\left(h_{v^{\prime}}-h_{v}\right)} ;
\end{aligned}
$$

if $1-\mathcal{P}_{0}-\mathcal{P}_{1}$ is applied, the bound improves by a factor $\left(\left|\mu_{h_{v}}\right| \gamma^{-h_{v}}\right)^{2} \leq$ $\gamma^{\frac{3}{2}\left(h_{v^{\prime}}-h_{v}\right)}$.

By suitably modifying the analysis leading to the bound (77), we can derive a bound for all the Schwinger functions and get a relatively simple tree expansion for their removed cutoffs limit. We shall here consider in detail the Schwinger functions with $n^{J}=0$, at fixed non coinciding points; we shall get a bound sufficient to prove two of the OSa, the boundedness and the cluster property. Since relativistic invariance is obvious by construction, to complete the proof of OSa there will remain to prove only positive definiteness.

Given a set $\underline{\mathbf{x}}=\left\{\mathbf{x}_{1}, \ldots, \mathbf{x}_{k}\right\}$ of $k$ (an even integer) space-time points, such that $\delta \equiv \min _{\mathbf{x} \neq \mathbf{y} \in \underline{\mathbf{x}}}|\mathbf{x}-\mathbf{y}|>0$, and a set $\underline{\omega}=\left\{\omega_{1}, \ldots, \omega_{k}\right\}$ of $\omega$ indices, the $k$-points Schwinger function $S_{k, \underline{\omega}}(\underline{\mathbf{x}})$ is defined as the $k$-th order functional derivative of the generating function (69) with respect to $\varphi_{\mathbf{x}_{1}, \omega_{1}}^{+}, \ldots, \varphi_{\mathbf{x}_{k / 2}, \omega_{k / 2}}^{+}$and $\varphi_{\mathbf{x}_{k / 2+1}, \omega_{k / 2+1}}^{-}, \ldots, \varphi_{\mathbf{x}_{k}, \omega_{k}}^{-}$at $J=\varphi=0$, see (17) and item 2) in Theorem 1. By using (69), we can write

$$
S_{k, \underline{\omega}}(\underline{\mathbf{x}})=\lim _{|h|, N \rightarrow \infty} \sum_{\pi(\underline{\mathbf{x}}, \underline{\omega})} \sum_{n=0}^{\infty} \sum_{j_{0}=h^{*}-1}^{N-1} \sum_{\underline{\omega}} \sum_{\tau \in \mathcal{T}_{j_{0}, n, k, 0}} \sum_{\substack{\mathbf{P} \in \mathcal{P} \\\left|P_{v_{0}}\right|=k}} S_{k, 0, \tau, \underline{\omega}}(\underline{\mathbf{x}}),
$$

where $\sum_{\pi(\underline{\mathbf{x}}, \underline{\omega})}$ denotes the sum over the permutations of the $\mathbf{x}$ and $\omega$ labels associated with the $k / 2$ endpoints of type $\varphi^{+}$, as well as those associated with the $k / 2$ endpoints of type $\varphi^{-}$. 
We need some extra definitions. Given a tree $\tau$ contributing to the r.h.s. of (81), we call $\tau^{*}$ the tree which is obtained from $\tau$ by erasing all the vertices which are not needed to connect the $k$ special endpoints (all of type $\varphi$ ). The endpoints of $\tau^{*}$ are the $k$ special endpoints of $\tau$, which we denote $v_{i}^{*}, i=1, \ldots, k$; with each of them a space-time point $\mathbf{x}_{i}$ is associated. Given a vertex $v \in \tau^{*}$, we shall call $\mathbf{x}_{v}^{*}$ the subset of $\underline{\mathbf{x}}$ made of all points associated with the endpoints following $v$ in $\tau^{*}$; we shall use also the definition $D_{v}=\max _{\mathbf{x}, \mathbf{y} \in \mathbf{x}_{v}^{*}}|\mathbf{x}-\mathbf{y}|$. Moreover, we shall call $s_{v}^{*}$ the number of branches following $v$ in $\tau^{*}, s_{v}^{*, 1}$ the number of branches containing only one endpoint and $s_{v}^{*, 2}=s_{v}^{*}-s_{v}^{*, 1}$. Note that $\mathbf{x}_{v}^{*} \subset \mathbf{x}_{v}$ and $s_{v}^{*} \leq s_{v}$.

The bound of $S_{k, 0, \tau, \underline{\omega}}(\underline{\mathbf{x}})$ can be obtained by slightly modifying the procedure described in detail in $\S 3$ of [BM1], which allowed us to prove the integral estimate (77), in order to take into account the fact that the points in $\underline{\mathbf{x}}$ are not integrated. First of all, we note that it is possible to extract a factor $e^{-c^{\prime} \sqrt{\gamma^{h_{v}} D_{v}}}$ for each non trivial (that is with $s_{v}^{*} \geq 2$, n.t. in the following) vertex $v \in \tau^{*}$, by partially using the decaying factors $e^{-c \sqrt{\gamma^{j}|\mathbf{x}-\mathbf{y}|}}$ appearing in the bounds (64), which are used for the propagators of the spanning tree $T_{\tau}=\bigcup_{v} T_{v}$ of $\tau$ (see (3.81) of [BM1]); we can indeed use the bound

$$
e^{-c \sqrt{\gamma^{h}|\mathbf{x}|}} \leq e^{-\frac{c}{2} \sqrt{\gamma^{h}|\mathbf{x}|}} \cdot e^{-c^{\prime} \sum_{j=-\infty}^{h} \sqrt{\gamma^{j}|\mathbf{x}|}} \quad, \quad c^{\prime}=\frac{c}{2 \sum_{j=0}^{\infty} \gamma^{-j / 2}}
$$

and the remark that, given a n.t. $v \in \tau^{*}$, there is a subtree $T_{v}^{*}$ of $T_{\tau}$, connecting the points in $\mathbf{x}_{v}^{*}$ (together with a subset of the internal points in $\mathbf{x}_{v}$ ), made of propagators of scale $j \geq h_{v}$. It follows that, given two points $\mathbf{x}, \mathbf{y} \in \mathbf{x}_{v}^{*}$, such that $D_{v}=|\mathbf{x}-\mathbf{y}|$, there is a path connecting $\mathbf{x}$ and $\mathbf{y}$, made of propagators in $T_{v}^{*}$, whose length is at least $D_{v}$; the decomposition of the decaying factors in the r.h.s. of (82) allows us to extract, for each of these propagators, a factor $e^{-c^{\prime} \sqrt{\gamma^{h_{v}}|\mathbf{x}|}}$ and the product of these factors can be bounded by $e^{-c^{\prime} \sqrt{\gamma^{h_{v} D_{v}}}}$.

Note that, after this operation, there will remain a factor $e^{-(c / 2) \sqrt{\gamma^{j}|\mathbf{x}-\mathbf{y}|}}$ for each propagator of $T_{\tau}$, to be used for the integration over the internal vertices. Moreover, there will be $1+\sum_{v \in \tau^{*}}\left(s_{v}^{*}-1\right)=k$ integrations less to do; by suitably choosing them, the lacking integrations produce in the bound an extra factor $\prod_{v \in \tau^{*}} \gamma^{2 h_{v}\left(s_{v}^{*}-1\right)} L^{-2}$ so that we get

$$
\begin{aligned}
& \left|S_{k, 0, \tau, \underline{\omega}}(\underline{\mathbf{x}})\right| \leq C^{k}\left(C \bar{\lambda}_{j_{0}}\right)^{n} \gamma^{-j_{0}(-2+k / 2)}\left[\prod_{n . t . v \in \tau^{*}} \gamma^{2 h_{v}\left(s_{v}^{*}-1\right)} e^{-c^{\prime} \sqrt{\gamma^{h_{v} D_{v}}}}\right] . \\
& \cdot \prod_{i=1}^{k} \frac{\gamma^{-h_{i}}}{\left(Z_{h_{i}}\right)^{1 / 2}} \prod_{\text {v not e.p. }}\left(\frac{Z_{h_{v}}}{Z_{h_{v}-1}}\right)^{\left|P_{v}\right| / 2} \gamma^{-d_{v}} .
\end{aligned}
$$

Let $E_{i}$ be the family of trivial vertices belonging to the branch of $\tau^{*}$ which connects $v_{i}^{*}$ with the higher non trivial vertex of $\tau^{*}$ preceding it; 
the definition of $s_{v}^{*, 1}$ and the fact that, by assumption, $1 / Z_{h_{i}} \leq \gamma^{h_{i} \eta}$, with $\eta \leq c \bar{\lambda}_{j_{0}}^{2}$, imply that, if $E=\cup_{i} E_{i}$,

$$
\prod_{i=1}^{k} \frac{\gamma^{-h_{i}}}{\left(Z_{h_{i}}\right)^{1 / 2}} \leq \prod_{v \in E} \gamma^{-(1-\eta / 2)} \prod_{n . t . v \in \tau^{*}} \gamma^{-h_{v}(1-\eta / 2) s_{v}^{*, 1}} .
$$

Let $v_{0}^{*}$ the first vertex following $v_{0}$ (the vertex immediately following the root of $\tau$, of scale $j_{0}+1$ ) with $s_{v}^{*} \geq 2$; then we have, if $k_{v}$ denotes the number of elements in $\mathbf{x}_{v}^{*}$ (hence $k_{v}=k$, if $v_{0} \leq v \leq v_{0}^{*}$ ),

$$
\left.\gamma^{-j_{0}(-2+k / 2}\right) \prod_{v_{0} \leq v<v_{0}^{*}} \gamma^{-d_{v}}=\gamma^{-h_{v_{0}^{*}}\left(-2+k_{v_{0}^{*}} / 2\right)} \prod_{v_{0} \leq v<v_{0}^{*}} \gamma^{-\tilde{d}_{v}}
$$

where we used the definition,

$$
\tilde{d}_{v}=d_{v}-\left(-2+\frac{k_{v}}{2}\right)=\frac{\left|P_{v}\right|-k_{v}}{2}
$$

note that $\tilde{d}_{v} \geq 1 / 2$, for any $v \in \tau^{*}$.

By inserting (84) and (85) in the r.h.s. of (83), we get

$$
\begin{aligned}
& \left|S_{k, 0, \tau, \underline{\omega}}(\underline{\mathbf{x}})\right| \leq C^{k}\left(C \bar{\lambda}_{j_{0}}\right)^{n}\left[\prod_{n . t . v \in \tau^{*}} e^{\left.-c^{\prime} \sqrt{\gamma^{h_{v} D_{v}}}\right]}\left[\prod_{v \text { not e.p. }}\left(\frac{Z_{h_{v}}}{Z_{h_{v}-1}}\right)^{\left|P_{v}\right| / 2}\right] .\right. \\
& \cdot\left[\prod_{\substack{v \notin \tau^{*} \\
\text { not e.p. }}} \gamma^{-d_{v}}\right]\left[\prod_{v \in E} \gamma^{-d_{v}-1+\eta / 2}\right]\left[\prod_{v_{0} \leq v<v_{0}^{*}} \gamma^{-\tilde{d}_{v}}\right] \cdot F_{\tau}
\end{aligned}
$$

where

$$
F_{\tau}=\gamma^{-h_{v_{0}^{*}}\left(-2+k_{v_{0}^{*}} / 2\right)} \prod_{n . t . v \in \tau^{*}} \gamma^{h_{v}\left[2\left(s_{v}^{*}-1\right)-(1-\eta / 2) s_{v}^{*, 1}\right]}\left[\prod_{\substack{v_{0}^{*} \leq v \in \tau^{*} \\ v \notin E}} \gamma^{-d_{v}}\right] .
$$

Given a n.t. vertex $v \in \tau^{*}$, let $s=s_{v}^{*}, s_{1}=s_{v}^{*, 1}, \tilde{s}=s-s_{1}$ and $v_{1}, \ldots, v_{\tilde{s}}$ the n.t. vertices immediately following $v$ in $\tau^{*}$. Note that $k_{v}=s_{1}+\sum_{i=1}^{\tilde{s}} k_{v_{i}}$; hence, given $\varepsilon>0$, we can write

$$
\begin{aligned}
& -\left(-2+\varepsilon+k_{v} / 2\right)+\left[2(s-1)-(1-\eta / 2) s_{1}\right]= \\
& =2-\varepsilon-k_{v} / 2+\varepsilon(s-1)+(2-\varepsilon)\left(s_{1}+\tilde{s}-1\right)-(1-\eta / 2) s_{1}= \\
& =-\frac{1}{2}\left(s_{1}+\sum_{i=1}^{\tilde{s}} k_{v_{i}}\right)+\varepsilon(s-1)+(2-\varepsilon) \tilde{s}+s_{1}(2-\varepsilon-1+\eta / 2)= \\
& =\varepsilon(s-1)+s_{1}(1 / 2-\varepsilon+\eta / 2)-\sum_{i=1}^{\tilde{s}}\left(-2+\varepsilon+k_{v_{i}} / 2\right) .
\end{aligned}
$$


This identity, applied to the vertex $v_{0}^{*}$, implies that, if $v_{1}, \ldots, v_{\tilde{s}}, \tilde{s}=s_{v_{0}^{*}}^{*}-$ $s_{v_{0}^{*}}^{*, 1}$, are the n.t. vertices immediately following $v_{0}^{*}$ in $\tau^{*}$, then

$$
\begin{aligned}
& \gamma^{-h_{v_{0}^{*}}\left(-2+k_{v_{0}^{*}} / 2\right)} \gamma^{h_{v_{0}^{*}}\left[2\left(s_{v_{0}^{*}}^{*}-1\right)-(1-\eta / 2) s_{v_{0}^{*}}^{*, 1}\right]}= \\
& =\gamma^{\varepsilon h_{v_{0}^{*}}} \gamma^{\alpha_{v_{0}^{*}} h_{v_{0}^{*}}} \prod_{i=1}^{\tilde{s}}\left[\gamma^{-h_{v_{i}}\left(-2+\varepsilon+k_{v_{i}} / 2\right)} \cdot \prod_{v \in \mathcal{C}_{i}} \gamma^{-2+\varepsilon+k_{v} / 2}\right],
\end{aligned}
$$

where $\mathcal{C}_{i}$ is the path connecting $v_{0}^{*}$ with $v_{i}$ in $\tau^{*}$ (not including $v_{i}$ ) and we used the definition

$$
\alpha_{v}=\varepsilon\left(s_{v}^{*}-1\right)+s_{v}^{*, 1}(1 / 2-\varepsilon+\eta / 2) .
$$

The presence of the factor $\gamma^{-h_{v_{i}}\left(-2+\varepsilon+k_{v_{i}} / 2\right)}$ for each vertex $v_{i}$ in the r.h.s. of (90) implies that an identity similar to (90) can be used for each n.t. vertex $v \in \tau^{*}$. It is then easy to show that

$$
F_{\tau}=\gamma^{\varepsilon h_{v_{0}^{*}}}\left[\prod_{n . t . v \in \tau^{*}} \gamma^{\alpha_{v} h_{v}}\right]\left[\prod_{v \in \tau^{*}, v \notin E} \gamma^{-\tilde{d}_{v}+\varepsilon}\right] .
$$

By inserting this equation in (87), we get

$$
\begin{aligned}
& \left|S_{k, 0, \tau, \underline{\omega}}(\underline{\mathbf{x}})\right| \leq C^{k}\left(C \bar{\lambda}_{j_{0}}\right)^{n} \gamma^{\varepsilon h_{v_{0}^{*}}}\left[\prod_{n . t . v \in \tau^{*}} \gamma^{\alpha h_{v}} e^{-c^{\prime} \sqrt{\gamma^{h v} D_{v}}}\right] . \\
& \cdot\left[\prod_{v \text { not e.p. }}\left(\frac{Z_{h_{v}}}{Z_{h_{v}-1}}\right)^{\left|P_{v}\right| / 2} \gamma^{-\bar{d}_{v}}\right],
\end{aligned}
$$

where

$$
\bar{d}_{v}= \begin{cases}\tilde{d}_{v} & \text { if } v_{0} \leq v<v_{0}^{*} \\ \tilde{d}_{v}-\varepsilon & \text { if } v \in \tau^{*}, v_{0}^{*} \leq v \notin E \\ d_{v}+1-\eta / 2 & \text { if } v \in E \\ d_{v} & \text { otherwise }\end{cases}
$$

Note that $\bar{d}_{v}>0$ for any $v \in \tau$, if $\varepsilon<1 / 2$; moreover, if this condition is satisfied, $\alpha_{v} \geq \varepsilon>0$, for any n.t. vertex $v \in \tau^{*}$, uniformly in $\bar{\lambda}_{j_{0}}$. Moreover, since by hypothesis $D_{v} \geq \delta>0$, there is $c_{0}$ such that

$$
\gamma^{\alpha_{v} h_{v}} e^{-c^{\prime} \sqrt{\gamma^{h_{v} D_{v}}}} \leq \sup _{x>0} x^{2 \alpha_{v}} e^{-c^{\prime} x \sqrt{\delta}} \leq\left(\frac{c_{0}}{\delta}\right)^{\alpha_{v}} \alpha_{v}^{2 \alpha_{v}}
$$

Note that

$$
\sum_{n . t . v \in \tau^{*}} \alpha_{v}=\frac{1}{2} k(1+\eta)-\varepsilon \leq \frac{1}{2} k(1+\eta) .
$$


Hence, by using (93) and (96), we get

$$
\begin{aligned}
& \left|S_{k, 0, \tau, \underline{\omega}}(\underline{\mathbf{x}})\right| \leq C^{k}(k !)^{1+\eta}\left(C \bar{\lambda}_{j_{0}}\right)^{n} \delta^{-[k(1+\eta) / 2-\varepsilon]+\alpha_{v_{0}^{*}}} . \\
& \cdot \gamma^{\left(\alpha_{v_{0}^{*}}+\varepsilon\right) h_{v_{0}^{*}}} e^{-c^{\prime} \sqrt{\gamma^{h} v_{0}^{*} D_{\underline{\mathbf{x}}}}}\left[\prod_{v \text { not e.p. }}\left(\frac{Z_{h_{v}}}{Z_{h_{v}-1}}\right)^{\left|P_{v}\right| / 2} \gamma^{-\bar{d}_{v}}\right] \text {, }
\end{aligned}
$$

where $D_{\underline{\mathbf{x}}}$ denotes the diameter of the set $\underline{\mathbf{x}}$.

Let us now observe that, since the vertex dimensions $\bar{d}_{v}$ are all strictly positive, if we insert the bound (97) in the r.h.s of (81), we can easily perform all the sums (by using the arguments explained, for instance, in [BM1]), once we have fixed the scale of the vertex $v_{0}^{*}$ and the values of $s_{v_{0}^{*}}^{*}$ and $s_{v_{0}^{*}}^{*, 1}$ (so that the value of $\alpha_{v_{0}^{*}}$ is fixed) and we can take the limit $-h, N \rightarrow \infty$. By using Theorem 5 and the remark that the bound (97) implies that the trees giving the main contribution to $S_{k, \underline{\omega}}(\underline{\mathbf{x}})$ are those with $\gamma^{h_{v_{0}^{*}}^{*}} D_{\underline{\mathbf{x}}}$ of order 1 , it is easy to prove that the limit can be expressed as an expansion similar to (81), with the sum over $j_{0}$ going from $-\infty$ to $+\infty$, the sum over $\tau$ including trees with endpoints of arbitrary scale (satisfying the usual constraints) and the values of $S_{k, 0, \tau, \underline{\omega}}(\underline{\mathbf{x}})$ modified in the following way:

1) in every endpoint there is the same constant $\lambda_{-\infty}$ in place of $\lambda_{h_{v}}$;

2) the constants $Z_{j}$, and $\mu_{j}$ are substituted everywhere by $\gamma^{-\eta_{z} j}$ and $\mu \gamma^{-\eta_{\mu} j}$, respectively, see (72);

$3)$ in the expansion which defines the constants $z_{j}$ and $s_{j}$ needed, respectively, in the definition of $\tilde{Z}_{j-1}(\mathbf{k})$ and $\mu_{j-1}(\mathbf{k})$, see (60), one has to make the same substitutions of items 1) and 2).

The bound (97) also implies the following one (valid for $C_{\varepsilon}|\lambda| \leq 1$, with $C_{\varepsilon} \rightarrow \infty$ as $\left.\varepsilon \rightarrow 1 / 2\right)$ :

$$
\begin{aligned}
& \left|S_{k, \underline{\omega}}(\underline{\mathbf{x}})\right| \leq C^{k}\left(C_{\varepsilon}|\lambda|\right)^{k / 2-1}(k !)^{2+\eta} \delta^{-[k(1+\eta) / 2-\varepsilon]} \sum_{s=2}^{k} \sum_{s_{1}=0}^{s} \delta^{\varepsilon(s-1)+s_{1}(1-2 \varepsilon+\eta) / 2} . \\
& \cdot \sum_{h=-\infty}^{+\infty} \gamma^{\left[\varepsilon s+s_{1}(1-2 \varepsilon+\eta) / 2\right] h} e^{-c^{\prime} \sqrt{\gamma^{h} D_{\underline{\mathbf{x}}}}} \leq \\
& \leq C^{k}\left(C_{\varepsilon}|\lambda|\right)^{k / 2-1}(k !)^{3+2 \eta} \delta^{-k(1+\eta) / 2} \sum_{s=2}^{k} \sum_{s_{1}=0}^{s}\left(\frac{\delta}{D_{\underline{\mathbf{x}}}}\right)^{\varepsilon s+s_{1}(1-2 \varepsilon+\eta) / 2}
\end{aligned}
$$

Since $\delta / D_{\underline{\mathbf{x}}} \leq 1$, the sum over $s$ and $s_{1}$ is bounded by $C k^{2}\left(\delta / D_{\underline{\mathbf{x}}}\right)^{2 \varepsilon}$; hence we get the bound

$$
\left|S_{k, \underline{\omega}}(\underline{\mathbf{x}})\right| \leq C^{k}\left(C_{\varepsilon}|\lambda|\right)^{k / 2-1}(k !)^{3+2 \eta} \delta^{-[k(1+\eta) / 2-2 \varepsilon]} \frac{1}{1+D_{\underline{\mathbf{x}}}^{2 \varepsilon}},
$$


which proves both the boundedness and the cluster property, see Appendix A.

In conclusion, we have proved the following result.

Theorem 6 If $\varepsilon_{1}$ is defined as in Theorem 5, there exists $\varepsilon_{2} \leq \varepsilon_{1}$ such that, if the normalization conditions (71) are satisfied and $|\lambda| \leq \varepsilon_{2}$, then the Schwinger functions $S_{k, \underline{\omega}}(\underline{\mathbf{x}})$ are well defined at non coinciding points and verify all the $O S$ axioms, possibly except the axiom of positive definiteness.

The positivity property will be proved in $\S 4$, together with the claim in item 4) of Theorem 1. Moreover, it is easy to derive from the previous bounds (see for instance [BM4] for the case $\mu=0$ ) the bound for the two point Schwinger functions (24). Finally, the previous arguments can be extended to prove that also the Schwinger functions with $n_{J}>0$ are well defined in the limit of removed cutoffs, so completing the proof of Theorem 1 , except for eq. (25), which will be proved in $\S 2.5$ below.

\subsection{Bounds for the Fourier transform of the Schwinger func- tions}

The main bound (77) can be also used to get bounds on the Fourier transform of the Schwinger functions at non zero external momenta; these bounds are uniform in the cutoffs and allow, in particular, to prove (by some obvious technicality, that we shall ship) that the removed cutoffs limit is well defined.

Here we shall only consider, as an example, the function $\widehat{G}_{\omega, \omega^{\prime}}^{2,1, N}(\mathbf{p} ; \mathbf{k})$ in the massless case.

By using (69), we can write

$$
\widehat{G}_{\omega, \omega^{\prime}}^{2,1, N}(\mathbf{p} ; \mathbf{k})=\sum_{n=0}^{\infty} \sum_{j_{0}=h-1}^{N-1} \sum_{\tau \in \mathcal{T}_{j_{0}, n, 2,1}} \sum_{\substack{\mathbf{P} \in \mathcal{P} \\\left|v_{0}\right|=2}} \widehat{G}_{\tau}^{2,1}(\mathbf{p}, \mathbf{k}),
$$

with an obvious definition of $\widehat{G}_{\tau}^{2,1}(\mathbf{p}, \mathbf{k})$. Let us define, for any $\mathbf{k} \neq 0$, $h_{\mathbf{k}}=\min \left\{j: f_{j}(\mathbf{k}) \neq 0\right\}$ and suppose that $\mathbf{p}, \mathbf{k}, \mathbf{p}-\mathbf{k}$ are all different from 0 . It follows that, given $\tau$, if $h_{-}$and $h_{+}$are the scale indices of the $\psi$ fields belonging to the endpoints associated with $\varphi^{+}$and $\varphi^{-}$, while $h_{J}$ denotes the scale of the endpoint of type $J, \widehat{G}_{\tau}^{2,1}(\mathbf{p}, \mathbf{k})$ can be different from 0 only if $h_{-}=h_{\mathbf{k}}, h_{\mathbf{k}}+1, h_{+}=h_{\mathbf{k}-\mathbf{p}}, h_{\mathbf{k}-\mathbf{p}}+1$ and $h_{J} \geq h_{\mathbf{p}}-\log _{\gamma} 2$. Moreover, if $\mathcal{T}_{j_{0}, n, \mathbf{p}, \mathbf{k}}$ denotes the set of trees satisfying the previous conditions and $\tau \in \mathcal{T}_{j_{0}, n, \mathbf{p}, \mathbf{k}},\left|\widehat{G}_{\tau}^{2,1}(\mathbf{p}, \mathbf{k})\right|$ can be bounded by $\int d \mathbf{z} d \mathbf{x}\left|G_{\tau}^{2,1}(\mathbf{z} ; \mathbf{x}, \mathbf{y})\right|$. Hence, by using (77) and (72), we get

$$
\left|\widehat{G}_{\omega, \omega^{\prime}}^{2,1, N, h}(\mathbf{p} ; \mathbf{k})\right| \leq C \gamma^{-h_{\mathbf{k}}\left(1-\eta_{z} / 2\right)} \gamma^{-h_{\mathbf{k}-\mathbf{p}}\left(1-\eta_{z} / 2\right)} .
$$




$$
\cdot \sum_{n=0}^{\infty} \sum_{j_{0}=h-1}^{N-1} \sum_{\tau \in \mathcal{T}_{j_{0}, n, \mathbf{p}, \mathbf{k}}} \sum_{\substack{\mathbf{P} \in \mathcal{P} \\\left|P_{v_{0}}\right|=2}}(C|\lambda|)^{n} \prod_{\text {v not e.p. }} \gamma^{-d_{v}} .
$$

The bound of the r.h.s. of (101) could be easily performed by using the procedure described in $\S 3$ of [BM1], if $d_{v}$ were greater than 0 for any $v$; however, by looking at (78), one sees that this is not true. Given $\tau \in \mathcal{T}_{j_{0}, n, \mathbf{p}, \mathbf{k}}$, let $v_{0}^{*}$ the higher vertex preceding all three special endpoints and $v_{1}^{*} \geq v_{0}^{*}$ the higher vertex preceding either the two endpoints of type $\varphi$ (to be called $v_{\varphi,+}$ and $v_{\varphi,-}$ ) or one endpoint of type $\varphi$ and the endpoint of type $J$ (to be called $\left.v_{J}\right)$. It turns out that $d_{v}>0$, except for the vertices belonging to the path $\mathcal{C}^{*}$ connecting $v_{1}^{*}$ with $v_{0}^{*}$, where, if $\left|P_{v}\right|=4$ and $n_{v}^{J}=0$ or $\left|P_{v}\right|=2$ and $n_{v}^{J}=1, d_{v}=0$. Hence, we can perform as in $\S 3$ of [BM1] the sums over the scale and $P_{v}$ labels of $\tau$, only if we fix the scale indices $h_{0}^{*}$ and $h_{1}^{*}$ of $v_{0}^{*}$ and $v_{1}^{*}$, after multiplying by $\gamma^{-\delta\left(h_{1}^{*}-h_{0}^{*}\right)}$ the r.h.s. of $(101), \delta$ being any positive number. Of course, we have also to perform the sum over $h_{0}^{*}, h_{1}^{*}$ of $\gamma^{\delta\left(h_{1}^{*}-h_{0}^{*}\right)}$, which is divergent, if we proceed exactly in this way.

In order to solve this problem, we note that, if $v \notin \mathcal{C}^{*}, d_{v}-1 / 4>0$. Hence, before performing the sums over the scale and $P_{v}$ labels, we can extract from each $\gamma^{-d_{v}}$ factor associated with the vertices belonging to the paths connecting the three special endpoints with $v_{0}^{*}$ or $v_{1}^{*}$, a $\gamma^{-1 / 4}$ piece, to be used to perform safely the sums over $h_{0}^{*}, h_{1}^{*}$ in the following way.

Let us consider first the family $\mathcal{T}_{j_{0}, n, \mathbf{p}, \mathbf{k}}^{(1)}$ of trees such that the two special endpoints following $v_{1}^{*}$ are $v_{\varphi,+}$ and $v_{\varphi,-}$ and let us suppose that $|\mathbf{k}| \geq|\mathbf{k}-\mathbf{p}|$. In this case, before doing the sums over the the scale and $P_{v}$ labels, we fix also the scale $h_{J}$ of $v_{J}$. We get, if $h_{J}^{*} \equiv \max \left\{h_{\mathbf{p}}+2, h_{0}^{*}+1\right\}$ :

$$
\begin{aligned}
& \sum_{n=0}^{\infty} \sum_{j_{0}=h-1}^{N-1} \sum_{\tau \in \mathcal{T}_{j_{0}, n, \mathbf{p}, \mathbf{k}}^{(1)}} \sum_{\substack{\mathbf{p} \in \mathcal{P} \\
\left|P_{0}\right|=2}}(C|\lambda|)^{n} \prod_{\mathrm{v} \text { not e.p. }} \gamma^{-d_{v}} \leq \\
& \leq C \sum_{h_{1}^{*}=-\infty}^{h_{\mathbf{k}-\mathbf{p}}} \sum_{h_{0}^{*}=-\infty}^{h_{1}^{*}} \sum_{h_{J}=h_{J}^{*}}^{+\infty} \gamma^{\delta\left(h_{1}^{*}-h_{0}^{*}\right)} \gamma^{-\frac{1}{4}\left[\left(h_{\mathbf{k}}-h_{1}^{*}\right)+\left(h_{\mathbf{k}-\mathbf{p}}-h_{1}^{*}\right)+\left(h_{J}-h_{0}^{*}\right)\right]},
\end{aligned}
$$

and it is easy to prove that the r.h.s. of (102) is bounded by $C \gamma^{\delta\left(h_{\mathbf{k}}-h_{\mathbf{p}}\right)}$, if $\delta \leq 1 / 8$. If $|\mathbf{k}-\mathbf{p}| \geq|\mathbf{k}|$, we get a similar result, with $h_{\mathbf{k}-\mathbf{p}}$ in place of $h_{\mathbf{k}}$.

Let us consider now the family $\mathcal{T}_{j_{0}, n, \mathbf{p}, \mathbf{k}}^{(2,+)}$ of trees such that the two special endpoints following $v_{1}^{*}$ are $v_{J}$ and $v_{\varphi,+}$. We get, if $h_{J}^{*} \equiv \max \left\{h_{\mathbf{p}}+2, h_{1}^{*}+1\right\}$ and $\bar{h}_{0}=\min \left\{h_{\mathbf{k}-\mathbf{p}}, h_{1}^{*}\right\}$ :

$$
\begin{aligned}
& \sum_{n=0}^{\infty} \sum_{j_{0}=h-1}^{N-1} \sum_{\tau \in \mathcal{T}_{j_{0}, n, \mathbf{p}, \mathbf{k}}^{(1,+)}} \sum_{\substack{\mathbf{P} \in \mathcal{P} \\
\left|v_{0}\right|=2}}(C|\lambda|)^{n} \prod_{\mathrm{v} \text { not e.p. }} \gamma^{-d_{v}} \leq \\
& \leq C \sum_{h_{1}^{*}=-\infty}^{h_{\mathbf{k}}} \sum_{h_{0}^{*}=-\infty}^{\bar{h}_{0}} \sum_{h_{J}=h_{J}^{*}}^{+\infty} \gamma^{\delta\left(h_{1}^{*}-h_{0}^{*}\right)} \gamma^{-\frac{1}{4}\left[\left(h_{\mathbf{k}}-h_{1}^{*}\right)+\left(h_{\mathbf{k}-\mathbf{p}}-h_{0}^{*}\right)+\left(h_{J}-h_{1}^{*}\right)\right]},
\end{aligned}
$$


and it is easy to prove that, if $\delta \leq 1 / 8$, the r.h.s. of (103) is bounded by $C \gamma^{\delta\left(h_{\mathbf{k}}-h_{\mathbf{k}-\mathbf{p}}\right)}$, if $|\mathbf{k}| \geq|\mathbf{k}-\mathbf{p}|$, by a constant, otherwise. The family $\mathcal{T}_{j_{0}, n, \mathbf{p}, \mathbf{k}}^{(2,-)}$ of trees such that the two special endpoints following $v_{1}^{*}$ are $v_{J}$ and $v_{\varphi,-}$ can be treated in a similar way and one obtains a bound $C \gamma^{\delta\left(h_{\mathbf{k}-\mathbf{p}}-h_{\mathbf{k}}\right)}$, if $|\mathbf{k}-\mathbf{p}| \geq|\mathbf{k}|$, or a constant, otherwise.

By putting together all these bounds, we get, for any positive $\delta \leq 1 / 8$ :

$$
\begin{aligned}
& \left|\widehat{G}_{\omega, \omega^{\prime}}^{2,1, N, h}(\mathbf{p} ; \mathbf{k})\right| \leq \frac{C_{\delta}}{|\mathbf{k}|^{1-\eta_{z}}|\mathbf{k}-\mathbf{p}|^{1-\eta_{z}}} . \\
& {\left[\left(\frac{|\mathbf{k}|}{|\mathbf{p}|}\right)^{\delta}+\left(\frac{|\mathbf{k}-\mathbf{p}|}{|\mathbf{p}|}\right)^{\delta}+\left(\frac{|\mathbf{k}|}{|\mathbf{k}-\mathbf{p}|}\right)^{\delta}+\left(\frac{|\mathbf{k}-\mathbf{p}|}{|\mathbf{k}|}\right)^{\delta}\right],}
\end{aligned}
$$

with $C_{\delta} \rightarrow \infty$ as $\delta \rightarrow 0$.

\subsection{Calculation of $\widehat{G}_{\omega}^{2}(\mathbf{k})$ in the massless case}

We want now to discuss the structure of the limit $-h, N \rightarrow \infty$ of the interacting propagator $\widehat{G}_{\omega}^{2, N, h}(\mathbf{k})$ for $\mu=0$.

By using (69), we can write

$$
\widehat{G}_{\omega}^{2, N, h}(\mathbf{k})=\sum_{n=0}^{\infty} \sum_{j_{0}=h-1}^{N-1} \sum_{\tau \in \mathcal{T}_{j_{0}, n, 2,0}} \sum_{\substack{\mathbf{P} \in \mathcal{P} \\\left|P_{v_{0}}\right|=2}} \widehat{G}_{\tau}^{2}(\mathbf{k}),
$$

with an obvious definition of $\widehat{G}_{\tau}^{2}(\mathbf{k})$.

Let us define $h_{\mathbf{k}}$ as in $\S 2.4$ and suppose that $\mathbf{k} \neq 0$. It follows that, given $\tau$, if $h_{-}$and $h_{+}$are the scale indices of the $\psi$ fields belonging to the endpoints associated with $\varphi^{+}$and $\varphi^{-}, \widehat{G}_{\tau}^{2}(\mathbf{k})$ can be different from 0 only if $h_{ \pm}=h_{\mathbf{k}}, h_{\mathbf{k}}+1$. Moreover, if $\mathcal{T}_{j_{0}, n, \mathbf{k}}$ denotes the set of trees satisfying the previous conditions and $\tau \in \mathcal{T}_{j_{0}, n, \mathbf{k}},\left|\widehat{G}_{\tau}^{2}(\mathbf{k})\right|$ can be bounded by $\int d \mathbf{x}\left|G_{\tau}^{2}(\mathbf{x}, \mathbf{y})\right|$. Hence, by using (77) and (72), we get

$$
\begin{aligned}
& \left|\widehat{G}_{\omega}^{2, N, h}(\mathbf{k})\right| \leq C \gamma^{-\left(h_{\mathbf{k}}-j_{0}\right)} \frac{\gamma^{-h_{\mathbf{k}}}}{Z_{h_{\mathbf{k}}}} . \\
& \cdot \sum_{n=0}^{\infty} \sum_{j_{0}=h-1}^{N-1} \sum_{\tau \in \mathcal{T}_{j_{0}, n, \mathbf{k}}} \sum_{\substack{\mathbf{P} \in \mathcal{P} \\
\left|P_{0}\right|=2}}(C|\lambda|)^{n} \prod_{\mathrm{v} \text { not e.p. }} \gamma^{-d_{v}},
\end{aligned}
$$

where $d_{v}>0$, except for the vertices belonging to the path connecting the root with $v^{*}$, the higher vertex preceding both the two special endpoints, where $d_{v}$ can be equal to 0 . These vertices can be regularized by using the factor $\gamma^{-\left(h_{\mathbf{k}}-j_{0}\right)}$ in the r.h.s. of (106); hence, by proceeding as in $\S 2.4$, we can easily perform the sum over the trees with a fixed value of the scale label $h^{*}$ of $v^{*}$ and we get the bound

$$
\left|\widehat{G}_{\omega}^{2, N, h}(\mathbf{k})\right| \leq C \frac{\gamma^{-h_{\mathbf{k}}}}{Z_{h_{\mathbf{k}}}} \sum_{h^{*}=-\infty}^{h_{\mathbf{k}}} \gamma^{-\left(h_{\mathbf{k}}-h^{*}\right) / 2} \leq C \frac{\gamma^{-h_{\mathbf{k}}}}{Z_{h_{\mathbf{k}}}} .
$$


By using Theorem 5, it is not hard to argue, as in $\S 2.3$, that the removed cutoffs limit $\widehat{G}_{\omega}^{2}(\mathbf{k})$ is well defined and is given by an expansion similar to (105), with the sum over $j_{0}$ going from $-\infty$ to $+\infty$ and the quantity $\widehat{G}_{\tau}^{2}(\mathbf{k})$ modified by substituting, in every endpoint, $\lambda_{j}$ with $\lambda_{-\infty}$, and, in every propagator, $Z_{j}$ with $\gamma^{-\eta j}, \eta \equiv \eta_{z}$; this property easily implies that $\widehat{G}_{\omega}^{2}(\gamma \mathbf{k})=$ $\gamma^{\eta-1} \widehat{G}_{\omega}^{2}(\mathbf{k})$. On the other hand, the symmetries of the model imply that there is a function $g(x, \lambda)$, defined for $x>0$ and $\lambda$ small enough, such that $\widehat{G}_{\omega}^{2}(\mathbf{k})=D_{\omega}^{-1}(\mathbf{k}) g(|\mathbf{k}|, \lambda)$; by the previous scaling property, $g(\gamma x, \lambda)=$ $\gamma^{\eta} g(x, \lambda)$. We want to show that $g(x, \lambda)=x^{\eta} f(\lambda)$, with $f(\lambda)$ independent of $x$.

To prove this claim, first of all note that $\widehat{G}_{\omega}^{2, N, h}(\mathbf{k})$ is independent of $\gamma$, since the cutoff function $C_{h, N}^{-1}(\mathbf{k})$ only depends on $\gamma_{0}$ and $\gamma \geq \gamma_{0}$, see $\S 1.2$. This property is then valid also for $\widehat{G}_{\omega}^{2}(\mathbf{k})$, hence for $g(x, \lambda)$. However, since the expansion heavily depends on $\gamma$, the value of $\eta$ is apparently a function of $\gamma$; we want to show that this is not true.

Note that, for any $\gamma$ and any integer $j, g\left(\gamma^{j}, \lambda\right)=\gamma^{j \eta(\gamma)} g(1, \lambda)$; it follows that, if there exist, given $\gamma_{1}$ and $\gamma_{2}$, two integers $j_{1}, j_{2}$, such that $\gamma_{1}^{j_{1}}=\gamma_{2}^{j_{2}}$, then $\eta\left(\gamma_{1}\right)=\eta\left(\gamma_{2}\right)$. Hence, given an interval $I=\left[\gamma_{0}, \bar{\gamma}\right]$ and $\gamma \in I$, the set $\left\{\gamma^{\prime} \in I: \eta\left(\gamma^{\prime}\right)=\eta(\gamma)\right\}$ is dense in $I$, as the set of rational numbers is dense in the interval $\left[\log _{\gamma} \gamma_{0}, \log _{\gamma} \bar{\gamma}\right]$. Since $\eta(\gamma)$ is obviously continuous in $\gamma$, it follows that it is constant.

Let us now put $g(x, \lambda)=x^{\eta} f(x, \lambda)$; we see immediately that $f(\gamma x, \lambda)=$ $f(x, \lambda)$. Hence, by varying $\gamma$ in the interval $[2,4]$ and by choosing $x=1 / \gamma$, we see that $f(1, \lambda)=f(x, \lambda)$, if $x \in[1 / 4,1 / 2]$. By using this equation, by varying $x$ in the interval $[1 / 4,1 / 2]$ and by choosing $\gamma=2$, we get also $f(1, \lambda)=f(x, \lambda)$, if $x \in[1 / 2,1]$. By proceeding in this way, it is easy to show that $f(1, \lambda)=f(x, \lambda)$, for any $x>0$.

The previous discussion and the fact that, in the expansion (106), $d_{v}>$ $1 / 4$ for any $v>v^{*}$, imply also that

$$
\widehat{G}_{\omega}^{2, N, h}(\mathbf{k})=\frac{|\mathbf{k}|^{\eta}}{D_{\omega}(\mathbf{k})}\left[f(\lambda)+O\left(|\mathbf{k}| \gamma^{-N}\right)^{1 / 4}\right] .
$$

\section{$3 \quad$ Ward-Takahashi Identities}

\subsection{Proof of Theorem 2}

In order to derive WTi in the massless case $\mu=0$ from the generating functional (13) (in the continuum limit $a=0$ ), it is convenient to introduce a cutoff function $\left[C_{h, N}^{\varepsilon}(\mathbf{k})\right]^{-1}$ equivalent to $\left[C_{h, N}(\mathbf{k})\right]^{-1}$ as far as the scaling features are concerned, but such that the support of $\left[C_{h, N}^{\varepsilon}(\mathbf{k})\right]^{-1}$ is the whole set $\mathcal{D}_{0}$ and $\lim _{\varepsilon \rightarrow 0}\left[C_{h, N}^{\varepsilon}(\mathbf{k})\right]^{-1}=\left[C_{h, N}(\mathbf{k})\right]^{-1}$; we refer to [BM2] $\S 2.2$ for its exact definition. We then substitute $\left[C_{h, N}(\mathbf{k})\right]^{-1}$ with $\left[C_{h, N}^{\varepsilon}(\mathbf{k})\right]^{-1}$ in the r.h.s. of (13) and perform the gauge transformation $\psi_{\mathbf{x}, \omega}^{ \pm} \rightarrow e^{ \pm \alpha_{\mathbf{x}, \omega}} \psi_{\mathbf{x}, \omega}^{ \pm}$ 
(equivalent to the usual phase and chiral transformations). The change in the cutoff function has the effect that the Lebesgue measure $d \widehat{\psi}^{[h, N]}$ is invariant under this transformation and we get the WTi (26), where, if $\left\langle.>_{h, N}\right.$ denotes the expectation with respect to measure $\mathcal{N}^{-1} P_{Z_{N}}(d \psi) e^{-\lambda_{N} Z_{N}^{2} V(\psi)}$ (see (14)-(16) for the definitions), $\widehat{\Delta}_{\omega, \omega^{\prime}}^{2,1, N}(\mathbf{p}, \mathbf{k})$ is the Fourier transform of

$$
\Delta_{\omega, \omega^{\prime}}^{2,1, N, h}(\mathbf{x} ; \mathbf{y}, \mathbf{z}) \stackrel{\text { def }}{=}\left\langle\psi_{\mathbf{y}, \omega^{\prime}}^{-} ; \psi_{\mathbf{z}, \omega^{\prime}}^{+} ; \delta T_{\mathbf{x}, \omega}\right\rangle_{h, N}
$$

where $\langle-;-;-\rangle_{h, n}$ denotes the truncated expectation with respect to the measure (14),

$$
\delta T_{\mathbf{x}, \omega} \stackrel{\text { def }}{=} \frac{Z_{N}}{L^{4}} \sum_{\substack{\mathbf{k}^{+}, \mathbf{k}^{-} \\ \mathbf{k}^{+} \neq \mathbf{k}^{-}}} e^{i\left(\mathbf{k}^{+}-\mathbf{k}^{-}\right) \mathbf{x}} C_{h, N ; \omega}^{\varepsilon}\left(\mathbf{k}^{+}, \mathbf{k}^{-}\right) \widehat{\psi}_{\mathbf{k}^{+}, \omega}^{+} \widehat{\psi}_{\mathbf{k}^{-}, \omega}^{-},
$$

and

$$
C_{h, N ; \omega}^{\varepsilon}\left(\mathbf{k}^{+}, \mathbf{k}^{-}\right)=\left[C_{h, N}^{\varepsilon}\left(\mathbf{k}^{-}\right)-1\right] D_{\omega}\left(\mathbf{k}^{-}\right)-\left[C_{h, N}^{\varepsilon}\left(\mathbf{k}^{+}\right)-1\right] D_{\omega}\left(\mathbf{k}^{+}\right) .
$$

Let us now suppose that $\mathbf{p}$ is fixed independently of $h$ and $N$, as well as $\mathbf{k}$, and that $\mathbf{p}, \mathbf{k}$ and $\mathbf{k}-\mathbf{p}$ are all different from 0 . This implies, in particular, that the condition $\tilde{\chi}(\mathbf{p})=1$ is satisfied if $|h|$ and $N$ are large enough and $\tilde{\chi}(\mathbf{p})$ is the function appearing in (31). Hence we can prove Theorem 2 by substituting in $(27) \widehat{R}_{\omega, \omega^{\prime}}^{2,1, N}(\mathbf{p}, \mathbf{k})$ with $\widetilde{\chi}(\mathbf{p}) \widehat{R}_{\omega, \omega^{\prime}}^{2,1, N, h}(\mathbf{p}, \mathbf{k})$, which is the Fourier transform of

$$
\left.\frac{\partial}{\partial J_{\mathbf{x}, \omega}} \frac{\partial^{2}}{\partial \varphi_{\mathbf{y}, \omega^{\prime}}^{+} \partial \varphi_{\mathbf{z}, \omega^{\prime}}^{-}} \mathcal{W}_{\Delta}\right|_{J=\varphi=0}
$$

where

$$
\begin{aligned}
e^{\mathcal{W}_{\Delta}(J, \varphi) \stackrel{\text { def }}{=}} \int \mathrm{d} P^{[h, N]}(\psi) \exp \left\{-\lambda_{N} V\left(\sqrt{Z_{N}} \psi\right)+\right. \\
\left.+\sum_{\omega} \int d \mathbf{x}\left[\varphi_{\mathbf{x}, \omega}^{+} \psi_{\mathbf{x}, \omega}^{-}+\psi_{\mathbf{x}, \omega}^{+} \varphi_{\mathbf{x}, \omega}^{-}\right]\right\} . \\
\cdot \quad \exp \left\{\sum_{\omega}\left[\bar{T}_{0, \omega}-\nu_{+, N} \bar{T}_{+, \omega}-\nu_{-, N} \bar{T}_{-, \omega}\right]\left(J, \sqrt{Z_{N}} \psi\right)\right\},
\end{aligned}
$$

with

$$
\begin{aligned}
\bar{T}_{0, \omega}(J, \psi) & =\frac{1}{L^{4}} \sum_{\mathbf{k}, \mathbf{p}} J_{\mathbf{p}, \omega} \widetilde{\chi}(\mathbf{p}) \frac{C_{h, N ; \omega}^{\varepsilon}(\mathbf{k}, \mathbf{k}-\mathbf{p})}{D_{\omega}(\mathbf{p})} \widehat{\psi}_{\mathbf{k}, \omega}^{+} \widehat{\psi}_{\mathbf{k}-\mathbf{p}, \omega}^{-} \equiv \frac{1}{L^{2}} \sum_{\mathbf{p} \neq 0} J_{\mathbf{p}, \omega} \delta \rho_{\mathbf{p}, \omega}, \\
\bar{T}_{ \pm, \omega}(J, \psi) & =\frac{1}{L^{4}} \sum_{\mathbf{k}, \mathbf{p}} J_{\mathbf{p}, \omega} \widetilde{\chi}(\mathbf{p}) \frac{D_{ \pm \omega}(\mathbf{p})}{D_{\omega}(\mathbf{p})} \widehat{\psi}_{\mathbf{k}, \pm \omega}^{+} \widehat{\psi}_{\mathbf{k}-\mathbf{p}, \pm \omega}^{-}
\end{aligned}
$$


The coefficients $\nu_{ \pm, N}$ will be fixed by the requirement that (29) holds. A crucial role in the analysis is played by the function

$$
\Delta_{\omega}^{(i, j)}\left(\mathbf{k}^{+}, \mathbf{k}^{-}\right)=\frac{C_{h, N ; \omega}^{\varepsilon}\left(\mathbf{k}^{+}, \mathbf{k}^{-}\right)}{D_{\omega}\left(\mathbf{k}^{+}-\mathbf{k}^{-}\right)} \widehat{g}_{\omega}^{(i)}\left(\mathbf{k}^{+}\right) \widehat{g}_{\omega}^{(j)}\left(\mathbf{k}^{-}\right),
$$

where $\widehat{g}_{\omega}^{(j)} \equiv \widehat{g}_{\omega, \omega}^{(j)}$. By proceeding as in $\S(4.2$.) of [BM2] (where only the case $N=0$ is considered), one can show that, if $\mathbf{p}=\mathbf{k}^{+}-\mathbf{k}^{-} \neq 0$ and $|\mathbf{p}| \geq 2 \gamma^{h+1}$ (which is true since $\tilde{\chi}(\mathbf{p})=1$ ):

1. if $h<i, j<N$, since $\left[C_{h, N}^{\varepsilon}\left(\mathbf{k}^{ \pm}\right)\right]^{-1}=1$,

$$
\Delta_{\omega}^{(i, j)}\left(\mathbf{k}^{+}, \mathbf{k}^{-}\right)=0
$$

2. if $h<j \leq N$,

$$
\Delta_{\omega}^{(N, j)}\left(\mathbf{k}^{+}, \mathbf{k}^{-}\right)=\frac{\mathbf{p}}{D_{\omega}(\mathbf{p})} \mathbf{S}_{\omega}^{(j)}\left(\mathbf{k}^{+}, \mathbf{k}^{-}\right)
$$

where $\mathbf{S}_{\omega}^{(j)}\left(\mathbf{k}^{+}, \mathbf{k}^{-}\right) \stackrel{\text { def }}{=}\left(S_{\omega, 0}^{(j)}\left(\mathbf{k}^{+}, \mathbf{k}^{-}\right), S_{\omega, 1}^{(j)}\left(\mathbf{k}^{+}, \mathbf{k}^{-}\right)\right)$is a vector of smooth functions such that

$$
\left|\partial_{\mathbf{k}^{+}}^{m_{+}} \partial_{\mathbf{k}^{-}}^{m_{-}} S_{\omega, i}^{(j)}\left(\mathbf{k}^{+}, \mathbf{k}^{-}\right)\right| \leq C_{m_{+}+m_{-}} \frac{\gamma^{-N\left(1+m_{+}\right)} \gamma^{-j\left(1+m_{-}\right)}}{Z_{N} Z_{j-1}} ;
$$

3. if $h \leq i \leq N$,

$$
\left|\Delta_{\omega}^{(i, h)}\left(\mathbf{k}^{+}, \mathbf{k}^{-}\right)\right| \leq C \gamma^{-(i-h)} \frac{\gamma^{-h-i}}{Z_{i-1} Z_{N}}
$$

4. if $i=j=h$,

$$
\Delta_{\omega}^{(h, h)}\left(\mathbf{k}^{+}, \mathbf{k}^{-}\right)=0 .
$$

Note that, in the r.h.s. of (119), there is apparently a $Z_{N} / Z_{h-1}$ factor missing, but the bound can not be improved; this is a consequence of the fact that $\tilde{Z}_{h-1}(\mathbf{k})=0$ for $|\mathbf{k}| \leq \gamma^{h-1}$, see eq. (63) of [BM2].

The multiscale integration of $\mathcal{W}_{\Delta}$ has been described in detail in $\S 4$ of [BM2] (of course the scale 0 has to be replaced with the scale $N$ ). After the integration of $\psi^{(N)}$ we get an expression like (42) and the terms linear in $J$ and quadratic in $\psi$ in the exponent will be denoted by $K_{J}^{(N-1)}\left(\sqrt{Z_{N-1}} \psi^{[h, N-1]}\right)$; we write $K_{J}^{(N-1)}=K_{J}^{(a, N-1)}+K_{J}^{(b, N-1)}$, where $K_{J}^{(a, N-1)}$ was obtained by the integration of $\bar{T}_{0}$ and $K_{J}^{(b, N-1)}$ from the integration of $\bar{T}_{ \pm}$. We can write $K_{J}^{(a, N-1)}$ as

$$
\begin{aligned}
& K_{J}^{(a, N-1)}\left(\sqrt{Z_{N-1}} \psi\right)=\sum_{\omega} Z_{N} \int d \mathbf{x} J_{\mathbf{x}, \omega}\left\{\bar{T}_{0, \omega}(J, \psi)+\right. \\
& \left.+\sum_{\tilde{\omega}} \int d \mathbf{y} d \mathbf{z}\left[F_{2, \omega, \tilde{\omega}}^{(N-1)}(\mathbf{x}, \mathbf{y}, \mathbf{z})+F_{1, \omega}^{(N-1)}(\mathbf{x}, \mathbf{y}, \mathbf{z}) \delta_{\omega, \tilde{\omega}}\right]\left[\psi_{\mathbf{y}, \tilde{\omega}}^{+} \psi_{\mathbf{z}, \tilde{\omega}}^{-}\right]\right\}
\end{aligned}
$$


where $F_{2, \omega, \tilde{\omega}}^{(N-1)}$ and $F_{1, \omega}^{(N-1)}$ are the analogous of eq. (132) of [BM2]; they represent the terms in which both or only one of the fields in $\delta \rho_{\mathbf{p},+}$, respectively, are contracted. Both contributions to the r.h.s. of (121) are dimensionally marginal; however, the regularization of $F_{1, \omega}^{(N-1)}$ is trivial, as it is of the form

$$
F_{1, \omega}^{(N-1)}\left(\mathbf{k}^{+}, \mathbf{k}^{-}\right)=\frac{\left[C_{h, N}\left(\mathbf{k}^{-}\right)-1\right] D_{\omega}\left(\mathbf{k}^{-}\right) Z_{N} \widehat{g}_{\omega}^{(N)}\left(\mathbf{k}^{+}\right)-u_{N}\left(\mathbf{k}^{+}\right)}{D_{\omega}\left(\mathbf{k}^{+}-\mathbf{k}^{-}\right)} G_{\omega}^{(2)}\left(\mathbf{k}^{+}\right)
$$

or the similar one, obtained exchanging $\mathbf{k}^{+}$with $\mathbf{k}^{-} ; u_{N}(\mathbf{k})=0$ if $|\mathbf{k}| \leq \gamma^{N}$ and $u_{N}(\mathbf{k})=1-f_{N}(\mathbf{k})$ for $|\mathbf{k}| \geq \gamma^{N}$. By the oddness of the propagator in the momentum, $G_{\omega}^{(2)}(0)=0$, hence we can regularize such term without introducing any local term, by simply rewriting it as

$$
\begin{gathered}
F_{1, \omega}^{(N-1)}\left(\mathbf{k}^{+}, \mathbf{k}^{-}\right)=\left[G_{\omega}^{(2)}\left(\mathbf{k}^{+}\right)-G_{\omega}^{(2)}(0)\right] . \\
\frac{\left[C_{h, N}\left(\mathbf{k}^{-}\right)-1\right] D_{\omega}\left(\mathbf{k}^{-}\right) Z_{N} \widehat{g}_{\omega}^{(N)}\left(\mathbf{k}^{+}\right)-u_{N}\left(\mathbf{k}^{+}\right)}{D_{\omega}\left(\mathbf{k}^{+}-\mathbf{k}^{-}\right)} .
\end{gathered}
$$

By using the symmetry property $(55), F_{2, \omega, \tilde{\omega}}^{(N-1)}$ can be written as

$$
F_{2, \omega, \tilde{\omega}}^{(N-1)}\left(\mathbf{k}^{+}, \mathbf{k}^{-}\right)=\frac{1}{D_{\omega}(\mathbf{p})}\left[p_{0} A_{0, \omega, \tilde{\omega}}\left(\mathbf{k}^{+}, \mathbf{k}^{-}\right)+p_{1} A_{1, \omega, \tilde{\omega}}\left(\mathbf{k}^{+}, \mathbf{k}^{-}\right)\right]
$$

where $A_{i, \omega, \tilde{\omega}}\left(\mathbf{k}^{+}, \mathbf{k}^{-}\right)$are functions such that, if we define

$$
\mathcal{L} F_{2, \omega, \tilde{\omega}}^{(N-1)}=\frac{1}{D_{\omega}(\mathbf{p})}\left[p_{0} A_{0, \omega, \tilde{\omega}}(0,0)+p_{1} A_{1, \omega, \tilde{\omega}}(0,0)\right]
$$

then

$$
\mathcal{L} F_{2, \omega, \omega}^{(N-1)}=Z_{N-1}^{3,+} \quad, \quad \mathcal{L} F_{2, \omega,-\omega}^{(N-1)}=\frac{D_{-\omega}(\mathbf{p})}{D_{\omega}(\mathbf{p})} Z_{N-1}^{3,-},
$$

where $Z_{N-1}^{3,+}$ and $Z_{N-1}^{3,-}$ are suitable real constants. Hence the local part of the marginal term in the second line of (121) is, by definition, equal to

$$
\sum_{\omega}\left[Z_{N} Z_{N-1}^{3,+} \bar{T}_{+, \omega}\left(J, \psi^{[h, N-1]}\right)+Z_{N} Z_{N-1}^{3,-} \bar{T}_{-, \omega}\left(J, \psi^{[h, N-1]}\right)\right] .
$$

The terms linear in $J$ and quadratic in $\psi$ obtained by the integration of $\bar{T}_{ \pm}$ have the form

$$
\begin{aligned}
& K_{J}^{(b, N-1)}\left(\sqrt{Z_{N-1}} \psi\right)=Z_{N} \frac{1}{L^{4}} \sum_{\mathbf{k}^{+}, \mathbf{p}} \tilde{\chi}(\mathbf{p}) J_{\mathbf{p}, \omega} \sum_{\omega, \tilde{\omega}} \widehat{\psi}_{\mathbf{k}^{+}, \tilde{\omega}}^{+} \widehat{\psi}_{\mathbf{k}^{+}-\mathbf{p}, \tilde{\omega}}^{-} \\
& \cdot\left[-\nu_{+, N} G_{\omega, \tilde{\omega}}^{(N)}\left(\mathbf{k}^{+}, \mathbf{k}^{+}-\mathbf{p}\right)-\nu_{-, N} \frac{D_{-\omega}(\mathbf{p})}{D_{\omega}(\mathbf{p})} G_{-\omega, \tilde{\omega}}^{(N)}\left(\mathbf{k}^{+}, \mathbf{k}^{+}-\mathbf{p}\right)\right](
\end{aligned}
$$

By using the symmetry property of the propagators, it is easy to show that $G_{\omega,-\omega}^{(N)}(0,0)=0$. Hence, if we regularize $(128)$ by subtracting $G_{\omega, \tilde{\omega}}^{(N)}(0,0)$ to 
$G_{\omega, \tilde{\omega}}^{(N)}\left(\mathbf{k}^{+}, \mathbf{k}^{+}-\mathbf{p}\right)$, we still get a local term of the form (127). Finally by collecting all the local term linear in $J$ we can write

$$
\begin{gathered}
\mathcal{L} K_{J}^{N-1}\left(\sqrt{Z_{N-1}} \psi^{[h, N-1]}\right)=\sum_{\omega}\left[Z_{N} \bar{T}_{0, \omega}\left(J, \psi^{[h, N-1]}\right)-\right. \\
\left.-\nu_{+, N-1} \bar{T}_{+, \omega}\left(J, \sqrt{Z_{N-2}} \psi^{[h, N-1]}\right)-\nu_{-, N-1} \bar{T}_{-, \omega}\left(J, \sqrt{Z_{N-2}} \psi^{[h, N-1]}\right)\right],
\end{gathered}
$$

where $Z_{N-2} \nu_{ \pm, N-1}=Z_{N-1}\left[\nu_{ \pm, N}-Z_{N-1}^{3, \pm}+\nu_{ \pm, N} G_{ \pm \omega, \pm \omega}^{(N)}(0,0)\right]$ (our definitions imply that $Z_{N-1}=Z_{N}$ ). The above integration procedure can be iterated with no important differences up to scale $h+1$. In particular, for all the marginal terms such that one of the fields in $\bar{T}_{0, \omega}$ in (121) is contracted at scale $j$, we put $\mathcal{R}=1$; in fact the second field has to be contracted at scale $h$ and, by (119), the extra factor $\gamma^{h-j}$ has the effect of automatically regularizing such contributions.

The above analysis implies that $\nu_{+, j}$ gets no contributions from trees with an endpoint of type $\nu_{-, k}, k>j$, and viceversa; moreover, if a tree has an endpoint corresponding to $\bar{T}_{0, \omega}$, this endpoint has scale index $N+1$. Hence we can write, for $h+1 \leq j \leq N-1$,

$$
\nu_{ \pm, j-1}=\nu_{ \pm, j}+\beta_{ \pm, \nu}^{j}\left(\lambda_{j}, \nu_{j} . ., \lambda_{N}, \nu_{N}\right)
$$

with

$$
\beta_{ \pm, \nu}^{j}\left(\lambda_{j}, \nu_{j} . ., \lambda_{N}, \nu_{N}\right)=\beta_{ \pm, \nu}^{j}\left(\lambda_{j}, . ., \lambda_{N}\right)+\sum_{j^{\prime}=j}^{N} \nu_{ \pm, j^{\prime}} \beta_{ \pm, \nu}^{j, j^{\prime}}\left(\lambda_{j}, . ., \lambda_{N}\right)
$$

and, given a positive $\vartheta<1 / 4$,

$$
\left|\beta_{ \pm, \nu}^{j}\left(\lambda_{j}, . ., \lambda_{N}\right)\right| \leq C \bar{\lambda}_{j} \gamma^{-2 \vartheta(N-j)} \quad,\left|\beta_{ \pm, \nu}^{j, j^{\prime}}\left(\lambda_{j}, . ., \lambda_{N}\right)\right| \leq C \bar{\lambda}_{j}^{2} \gamma^{-2 \vartheta\left|j-j^{\prime}\right|} .
$$

We fix $\nu_{ \pm, N}$ so that

$$
\nu_{ \pm, N}=-\sum_{j=h+1}^{N} \beta_{ \pm, \nu}^{j}\left(\lambda_{j}, \nu_{j} . ., \lambda_{N}, \nu_{N}\right) .
$$

By a fixed point argument (see $\S 4.6$ of [BM4]), one can show that, if $\bar{\lambda}_{h}$ is small enough, it is possible to choose $\nu_{ \pm, N}$ so that

$$
\left|\nu_{\omega, j}\right| \leq c_{0} \bar{\lambda}_{h} \gamma^{-\vartheta(N-j)}
$$

for any $h+1 \leq j \leq N$.

The convergence of $\nu_{ \pm, N}$ as $|h|, N \rightarrow \infty$ is an easy consequence of the previous considerations. Moreover, from an explicit computation of (130), we get $\nu_{-}=\frac{\lambda}{4 \pi}+O\left(\lambda^{2}\right)$ and $\nu_{+}=c_{+} \lambda^{2}+O\left(\lambda^{3}\right)$ with $c_{+}<0$. 

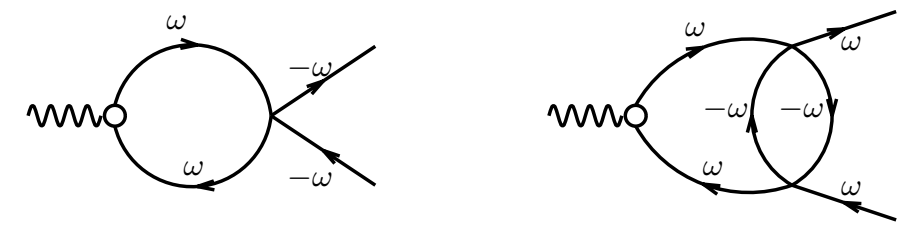

Figure 4: : Graphical representation of the lowest order contribution to $\nu^{-}$ and to $\nu^{+}$; the small circle represent the operator $C$ (111)

The convergence of $\widehat{G}_{\omega, \omega^{\prime}}^{2,1, N, h}(\mathbf{p} ; \mathbf{k})$ was discussed in $\S 2.4$. Hence, to complete the proof of Theorem 2, we have to prove that $\tilde{\chi}(\mathbf{p}) \widehat{R}_{\omega, \omega^{\prime}}^{2,1, N}(\mathbf{p}, \mathbf{k}) \rightarrow 0$ , if $\mathbf{p}, \mathbf{k}$ and $\mathbf{k}-\mathbf{p}$ are all different from 0 . In fact, since $\widetilde{\chi}(\mathbf{p})=1$ for $\mathbf{p} \neq 0$ and $|h|, N$ large enough, this implies (29).

This result can be obtained by a simple extension of the arguments given in $\S 2.4$ to prove that $\widehat{G}_{\omega, \omega^{\prime}}^{2,1, N, h}(\mathbf{p} ; \mathbf{k})$ is bounded uniformly in $h$ and $N$. In fact, $\widetilde{\chi}(\mathbf{p}) \widehat{R}_{\omega, \omega^{\prime}}^{2,1, h}(\mathbf{p}, \mathbf{k})$ can be written by a sum of trees essentially identical to the ones for $\widehat{G}_{\omega, \omega^{\prime}}^{2,1, h}$, with the only important difference that there are three different special endpoints associated to the field $J$, corresponding to the three different terms in (129); we call these endpoints of type $T_{0}, T_{+}, T_{-}$ respectively.

The sum over the trees such that the endpoint is of type $T_{ \pm}$can be bounded as in (101), the only difference being that, thanks to the bound (134), one has to multiply the r.h.s. by a factor $|\lambda| \gamma^{-\vartheta\left(N-h_{J}\right)}$, which has to be inserted also in the r.h.s. of the bounds (102) and (103). Hence, it is easy to see that the contributions of these trees vanishes as $N \rightarrow \infty$.

Let us now consider the trees with an endpoint of type $T_{0}$. In this case there are two possibilities. The first is that the fields of the $T_{0}$ endpoint are contracted at scale $j, N$; this implies that the sum over $h_{J}$ is missing in the r.h.s. of the bounds (102) and (103) and $h_{J}=N$. Hence it is easy to see that the sum over such trees goes to 0 as $N \rightarrow \infty$. The second possibility is that the fields of the $T_{0}$ endpoint are contracted at scale $j, h$; this implies that the sum over $j_{0}$ is missing in the r.h.s. of (101) and $j_{0}=h$. Since $d_{v}-1 / 4>0$ for all vertices belonging to the path connecting the root to the vertex $v_{0}^{*}$, we can add a factor $\gamma^{\left.-\left(j_{0}-h\right)\right) / 4}$ to the r.h.s. of the bounds (102) and (103), which then go to 0 as $h \rightarrow-\infty$.

\section{Schwinger-Dyson equations and new anomalies}

\subsection{Proof of Theorem 3}

In this section we study the last addend of the Schwinger-Dyson equation (31), so proving Theorem 3; the analysis rests heavily on $\S 4$ of [BM4].

Let us consider a fixed finite $\mathbf{k}$ and let us define its scale $h_{\mathbf{k}}$ as in $\S 2.4$; 
then, if $-h$ and $N$ are large enough, $\widehat{g}_{\omega}^{N, h}(\mathbf{k})=\widehat{g}_{\omega}(\mathbf{k})$. We start by putting (see $\S 4.1$ of $[\mathrm{BM} 4])$ :

$$
\widetilde{G}_{\varepsilon, \omega}^{2, N, h}(\mathbf{k}) \stackrel{\text { def }}{=} \widehat{g}_{\omega}(\mathbf{k}) \int \frac{d \mathbf{p}}{(2 \pi)^{2}} \widetilde{\chi}(\mathbf{p}) \frac{D_{\varepsilon \omega}(\mathbf{p})}{D_{-\omega}(\mathbf{p})} \widehat{R}_{\varepsilon \omega ; \omega}^{2,1, N, h}(\mathbf{p}, \mathbf{k})=\frac{\partial \mathcal{W}_{T, \varepsilon}}{\partial \widehat{\varphi}_{\mathbf{k}, \omega}^{+} \partial \widehat{J}_{\mathbf{k}, \omega}},
$$

where $\varepsilon= \pm$ and $\mathcal{W}_{T, \varepsilon}$ is defined (in the infinite volume limit) by the equation:

$$
\begin{aligned}
& e^{\mathcal{W}_{T, \varepsilon}(J, \varphi) \stackrel{\text { def }}{=}} \int \mathrm{d} P_{Z_{N}}(\psi) \exp \left\{-\lambda_{N} Z_{N}^{2} V(\psi) \int d \mathbf{x}\left[\varphi_{\mathbf{x}, \omega}^{+} \psi_{\mathbf{x}, \omega}^{[h, N]-}+\right.\right. \\
& \left.\left.+\psi_{\mathbf{x}, \omega}^{[h, N]+} \varphi_{\mathbf{x}, \omega}^{-}\right]+Z_{N}\left[T_{1}^{(\varepsilon)}-\nu_{+, N} T_{-\varepsilon}-\nu_{-, N} T_{\varepsilon}\right](\psi, J)\right\}
\end{aligned}
$$

with

$$
\begin{aligned}
T_{1}^{(\varepsilon)}(\psi, J) \stackrel{\text { def }}{=} & \int \frac{d \mathbf{p} d \mathbf{k}^{\prime}}{(2 \pi)^{4}} \tilde{\chi}(\mathbf{p}) \widehat{J}_{\mathbf{k}, \omega} \widehat{g}_{\omega}(\mathbf{k}) \frac{C_{\varepsilon \omega}\left(\mathbf{k}^{\prime}, \mathbf{k}^{\prime}-\mathbf{p}\right)}{D_{-\omega}(\mathbf{p})} \\
& \cdot \widehat{\psi}_{\mathbf{k}-\mathbf{p}, \omega}^{+} \widehat{\psi}_{\mathbf{k}^{\prime}, \varepsilon \omega}^{+} \widehat{\psi}_{\mathbf{k}^{\prime}-\mathbf{p}, \varepsilon \omega}^{-}, \\
T_{+}(\psi, J) \stackrel{\text { def }}{=} & \int \frac{d \mathbf{p} d \mathbf{k}^{\prime}}{(2 \pi)^{4}} \tilde{\chi}(\mathbf{p}) \widehat{J}_{\mathbf{k}, \omega} \widehat{g}_{\omega}(\mathbf{k}) \widehat{\psi}_{\mathbf{k}-\mathbf{p}, \omega}^{+} \widehat{\psi}_{\mathbf{k}^{\prime},-\omega}^{+} \widehat{\psi}_{\mathbf{k}^{\prime}-\mathbf{p},-\omega}^{-}, \\
T_{-}(\psi, J) \stackrel{\text { def }}{=} & \int \frac{d \mathbf{p} d \mathbf{k}^{\prime}}{(2 \pi)^{4}} \tilde{\chi}(\mathbf{p}) \widehat{J}_{\mathbf{k}, \omega} \widehat{g}_{\omega}(\mathbf{k}) \frac{D_{\omega}(\mathbf{p})}{D_{-\omega}(\mathbf{p})} \widehat{\psi}_{\mathbf{k}-\mathbf{p}, \omega}^{+} \widehat{\psi}_{\mathbf{k}^{\prime}, \omega}^{+} \widehat{\psi}_{\mathbf{k}^{\prime}-\mathbf{p}, \omega}^{-},
\end{aligned}
$$

and $\nu_{ \pm, N}$ are defined as in (133).

The calculation of $\widetilde{G}_{\varepsilon, \omega}^{2, N, h}(\mathbf{k})$ is done again via a multiscale expansion, very similar to the one described in $\S 4$ of [BM4]. The main differences are that here we are considering a quantity with two external lines, instead of four, and that the external momenta are on the scale $h_{\mathbf{k}}$, instead of the infrared cutoff scale $h$. However, the last remark implies that the integration of the fields of scale $j>h_{\mathbf{k}}+1$ differs from that discussed in [BM4] only for trivial scaling factors; in particular, there is no contribution to $\widetilde{G}_{\varepsilon, \omega}^{2, N, h}(\mathbf{k})$ associated with a tree, whose root has scale higher than $h_{\mathbf{k}}$.

Let us call $\overline{\mathcal{V}}^{(N-1)}\left(\psi^{[h, N-1]}\right)$ the sum over the terms linear in $J$, obtained after the integration of the field $\psi^{(N)}$; we put:

$$
\begin{aligned}
& \overline{\mathcal{V}}^{(N-1)}\left(\psi^{[h, N-1]}\right)=\overline{\mathcal{V}}_{a, 1}^{(N-1)}\left(\psi^{[h, N-1]}\right)+\overline{\mathcal{V}}_{a, 2}^{(N-1)}\left(\psi^{[h, N-1]}\right)+ \\
& \overline{\mathcal{V}}_{b, 1}^{(N-1)}\left(\psi^{[h, N-1]}\right)+\overline{\mathcal{V}}_{b, 2}^{(N-1)}\left(\psi^{[h, N-1]}\right),
\end{aligned}
$$

where $\overline{\mathcal{V}}_{a, 1}^{(N-1)}+\overline{\mathcal{V}}_{a, 2}^{(N-1)}$ is the sum of the terms in which the field $\widehat{\psi}_{\mathbf{k}-\mathbf{p}, \omega}^{+}$ appearing in the definition of $T_{1}^{(\varepsilon)}(\psi)$ or $T_{ \pm}(\psi)$ is contracted, $\overline{\mathcal{V}}_{a, 1}^{(N-1)}$ and $\overline{\mathcal{V}}_{a, 2}^{(N-1)}$ denoting the sum over the terms of this type containing a $T_{1}$ or a $T_{ \pm}$vertex, respectively; $\overline{\mathcal{V}}_{b, 1}^{(N-1)}+\overline{\mathcal{V}}_{b, 2}^{(N-1)}$ is the sum of the other terms, that 
is those where the field $\widehat{\psi}_{\mathbf{k}-\mathbf{p}, \omega}^{+}$is an external field, the index $i=1,2$ having the same meaning as before.

Let us consider first $\overline{\mathcal{V}}_{a, 1}^{(N-1)}$; we shall still distinguish different group of terms, those where both fields $\widehat{\psi}_{\mathbf{k}^{\prime}, \varepsilon \omega}^{+}$and $\widehat{\psi}_{\mathbf{k}^{\prime}-\mathbf{p}, \varepsilon \omega}^{-}$are contracted, those where only one among them is contracted and those where no one is contracted. If no one of the fields $\widehat{\psi}_{\mathbf{k}^{\prime}, \varepsilon \omega}^{+}$and $\widehat{\psi}_{\mathbf{k}^{\prime}-\mathbf{p}, \varepsilon \omega}^{-}$is contracted, we can only have terms with at least four external lines; for the properties of $\Delta_{\varepsilon \omega}^{(i, j)}$ (see (115)), at least one of the fields $\widehat{\psi}_{\mathbf{k}^{\prime}, \varepsilon \omega}^{+}$and $\widehat{\psi}_{\mathbf{k}^{\prime}-\mathbf{p}, \varepsilon \omega}^{-}$must be contracted at scale $h$. If one of these terms has four external lines, hence it is marginal, it has the following form

$$
\begin{aligned}
& Z_{N} \int \frac{d \mathbf{p} d \mathbf{k}^{\prime}}{(2 \pi)^{4}} \widehat{\psi}_{\omega, \mathbf{k}-\mathbf{p}}^{+} G_{2}^{(N)}(\mathbf{k}-\mathbf{p}) \widehat{g}_{\omega}^{N, h}(\mathbf{k}-\mathbf{p}) . \\
& \cdot \widehat{J}_{\mathbf{k}, \omega} \widehat{g}_{\omega}(\mathbf{k}) \widetilde{\chi}(\mathbf{p}) \frac{C_{\varepsilon \omega}\left(\mathbf{k}^{\prime}, \mathbf{k}^{\prime}-\mathbf{p}\right)}{D_{-\omega}(\mathbf{p})} \widehat{\psi}_{\mathbf{k}^{\prime}, \varepsilon \omega}^{+} \widehat{\psi}_{\mathbf{k}^{\prime}-\mathbf{p}, \varepsilon \omega}^{-},
\end{aligned}
$$

where $G_{2}^{(N)}(\mathbf{k})$ is a suitable function which can be expressed as a sum of graphs with an odd number of propagators, hence it vanishes at $\mathbf{k}=0$. This implies that $G_{2}^{(N)}(0)=0$, so that we can regularize it without introducing any running coupling.

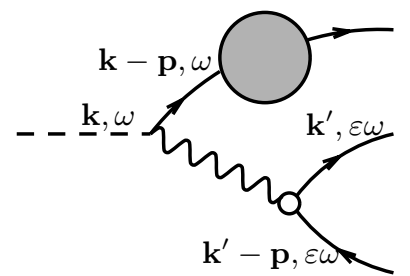

Figure 5: : Graphical representation of (139)

If both $\widehat{\psi}_{\mathbf{k}^{\prime}, \varepsilon \omega}^{+}$and $\widehat{\psi}_{\mathbf{k}^{\prime}-\mathbf{p}, \varepsilon \omega}^{-}$in $T_{1}^{(\varepsilon)}(\psi)$ are contracted, we get terms of the form (up to an integral over the external momenta)

$$
\widehat{J}_{\mathbf{k}, \omega} g_{\omega}(\mathbf{k}) \tilde{W}_{n+1}^{(N-1)}\left(\mathbf{k}, \mathbf{k}_{1}, . ., \mathbf{k}_{n}\right)\left(\sqrt{Z_{N}}\right)^{n-1} \prod_{i=1}^{n} \widehat{\psi}_{\mathbf{k}_{i}}^{\varepsilon_{i}},
$$

where $n$ is an odd integer. We want to define an $\mathcal{R}$ operation for such terms. There is apparently a problem, as the $\mathcal{R}$ operation involves derivatives and any term contributing to $\tilde{W}_{n+1}^{(N-1)}$ contains the $\Delta_{\varepsilon \omega}^{(N, N)}$ and the cutoff function $\tilde{\chi}(\mathbf{p})$. Hence one can worry about the derivatives of the factor $\tilde{\chi}(\mathbf{p}) \mathbf{p} D_{-\omega}(\mathbf{p})^{-1}$. However, as $\mathbf{k}$ is fixed independently from $N$ (and far enough from $\gamma^{N}$ ) and $\mathbf{k}-\mathbf{p}$ is fixed at scale $N$, then $|\mathbf{p}| \geq \gamma^{N-1} / 2$, so that we can freely multiply by a smooth cutoff function $\bar{\chi}(\mathbf{p})$ restricting $\mathbf{p}$ to the 
allowed region; this allows us to pass to coordinate space and shows that the $\mathcal{R}$ operation can be defined in the usual way. We define

$$
\begin{gathered}
\mathcal{L} \tilde{W}_{4}^{(N-1)}\left(\mathbf{k}, \mathbf{k}_{1}, \mathbf{k}_{2}, \mathbf{k}_{3}\right)=\tilde{W}_{4}^{(N-1)}(0, . ., 0), \\
\mathcal{L} \tilde{W}_{2}^{(N-1)}(\mathbf{k})=\tilde{W}_{2}^{(N-1)}(0)+\mathbf{k} \partial_{\mathbf{k}} \tilde{W}_{2}^{(N-1)}(0) .
\end{gathered}
$$

Note that by parity the first term in (142) is vanishing; this means that there are only marginal terms.

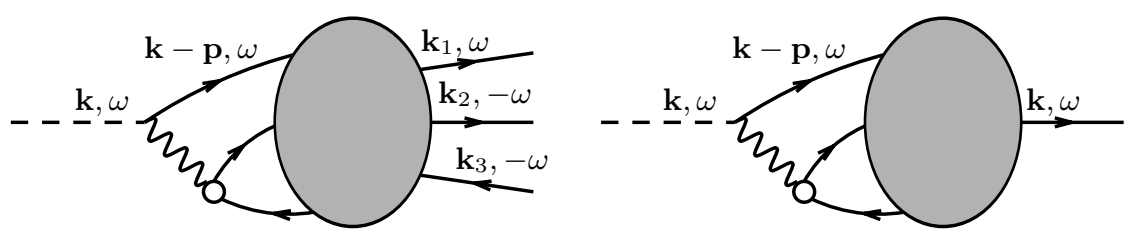

Figure 6: : Graphical representation of $\tilde{W}_{4}^{(-1)}$ and $\tilde{W}_{2}^{(-1)}$

If only one among the fields $\widehat{\psi}_{\mathbf{k}^{\prime}, \varepsilon \omega}^{+}$and $\widehat{\psi}_{\mathbf{k}^{\prime}-\mathbf{p}, \varepsilon \omega}^{-}$in $T_{1}(\psi)$ is contracted, we get terms with four external lines of the form (up to an integral over the external momenta):

$$
\begin{aligned}
& Z_{N} \widehat{g}_{\omega}(\mathbf{k}) \widehat{J}_{\mathbf{k}, \omega} \widehat{\psi}_{\mathbf{k}_{1}, \omega}^{+} \widehat{\psi}_{\mathbf{k}^{-}, \varepsilon \omega}^{-} \widehat{\psi}_{\mathbf{k}^{-}+\mathbf{k}-\mathbf{k}_{1}, \omega_{2}}^{+} \int d \mathbf{k}^{+} \tilde{\chi}\left(\mathbf{k}^{+}-\mathbf{k}^{-}\right) \cdot \\
& \cdot \widehat{g}_{\omega}^{N, h}\left(\mathbf{k}-\mathbf{k}^{+}+\mathbf{k}^{-}\right) G_{4}^{(N)}\left(\mathbf{k}^{+}, \mathbf{k}_{1}, \mathbf{k}+\mathbf{k}_{-}-\mathbf{k}_{1}\right) \cdot \\
& \cdot\left\{\frac{\left[C_{h, N}\left(\mathbf{k}^{-}\right)-1\right] D_{\varepsilon \omega}\left(\mathbf{k}^{-}\right) \widehat{g}_{\varepsilon \omega}^{N, h}\left(\mathbf{k}^{+}\right)}{D_{-\omega}\left(\mathbf{k}^{+}-\mathbf{k}^{-}\right)}-\frac{u_{N}\left(\mathbf{k}^{+}\right)}{D_{-\omega}\left(\mathbf{k}^{+}-\mathbf{k}^{-}\right)}\right\},
\end{aligned}
$$

or the similar one with the roles of $\mathbf{k}^{+}$and $\mathbf{k}^{-}$exchanged. Note that the indices $\omega_{1}$ and $\omega_{2}$ must satisfy the constraint $\omega_{1} \omega_{2}=\varepsilon$.

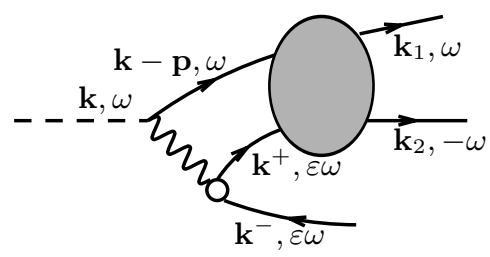

Figure 7: : Graphical representation of a single addend in (143)

The two terms in (143) must be treated differently, as concerns the regularization procedure. The first term is such that one of the external lines is associated with the operator $\left[C_{h, N}\left(\mathbf{k}^{-}\right)-1\right] D_{\varepsilon \omega}\left(\mathbf{k}^{-}\right) D_{-\omega}(\mathbf{p})^{-1}$. We define $\mathcal{R}=1$ for such terms; in fact, when such external line is contracted (and this can happen only at scale $h$ ), the factor $D_{\varepsilon \omega}\left(\mathbf{k}^{-}\right) D_{-\omega}(\mathbf{p})^{-1}$ produces an extra factor $\gamma^{h-N}$ in the bound, with respect to the dimensional one. The second 
term in (143) can be regularized as above, by subtracting the value of the kernel computed at zero external momenta, i.e. for $\mathbf{k}^{-}=\mathbf{k}=\mathbf{k}_{1}=0$. Note that such quantity vanishes, if the four $\omega$-indices are all equal, otherwise it is given by the product of the field variables times

$$
-Z_{N} \widehat{g}_{\omega}(\mathbf{k}) \widehat{J}_{\mathbf{k}, \omega} \int d \mathbf{k}^{+} \tilde{\chi}\left(\mathbf{k}^{+}\right) \widehat{g}_{\omega}^{N, h}\left(\mathbf{k}^{+}\right) G_{4}^{(N)}\left(\mathbf{k}^{+}, 0,0\right) \frac{u_{N}\left(\mathbf{k}^{+}\right)}{D_{-\omega}\left(\mathbf{k}^{+}\right)},
$$

and there is no singularity associated with the factor $D_{-\omega}\left(\mathbf{k}^{+}\right)^{-1}$, thanks to the support on scale $N$ of the propagator $\widehat{g}_{\omega}^{N, h}\left(\mathbf{k}^{+}\right)$. The terms with two external lines can be produced only if $\varepsilon=+1$ and can be treated in a similar way; they have the form

$$
\begin{aligned}
& \widehat{\psi}_{\mathbf{k}, \omega}^{-} \widehat{g}_{\omega}(\mathbf{k}) \widehat{J}_{\mathbf{k}, \omega} \int d \mathbf{k}^{+} \tilde{\chi}\left(\mathbf{k}^{+}-\mathbf{k}\right) G_{1}^{(N)}\left(\mathbf{k}_{+}\right) \\
& \cdot\left\{\frac{\left[C_{h, N}^{\varepsilon}(\mathbf{k})-1\right] D_{\varepsilon \omega}(\mathbf{k}) \widehat{g}_{\omega}^{(N)}\left(\mathbf{k}^{+}\right)}{D_{-\omega}\left(\mathbf{k}^{+}-\mathbf{k}\right)}-\frac{u_{N}\left(\mathbf{k}^{+}\right)}{D_{-\omega}\left(\mathbf{k}^{+}-\mathbf{k}\right)}\right\},
\end{aligned}
$$

where $G_{1}^{(N)}\left(\mathbf{k}_{+}\right)$is a smooth function of order 0 in $\lambda$. However, the first term in the braces is equal to 0 , since we keep $\mathbf{k}$ fixed and far from the cutoffs, hence $C_{h, N}^{\varepsilon}(\mathbf{k})-1=0$, and the second term can be regularized as above.

A similar (but simpler) analysis holds for the terms contributing to $\overline{\mathcal{V}}_{a, 2}^{(N-1)}$, which contain a vertex of type $T_{+}$or $T_{-}$and are of order $\lambda \nu_{ \pm}$. Now, the only thing to analyze carefully is the possible singularities associated with the factors $\tilde{\chi}(\mathbf{p})$ and $\mathbf{p} D_{-\omega}(\mathbf{p})^{-1}$. However, since in these terms the field $\widehat{\psi}_{\mathbf{k}-\mathbf{p}, \omega}^{+}$is contracted, $|\mathbf{p}| \geq \gamma^{N-1} / 2$; hence the regularization procedure can not produce bad dimensional bounds.

We will define $\tilde{z}_{N-1}^{(\varepsilon)}$ and $\tilde{\lambda}_{N-1}^{(\varepsilon)}$, so that (recall that $Z_{N-1}=Z_{N}$ )

$$
\begin{aligned}
& \mathcal{L}\left[\overline{\mathcal{V}}_{a, 1}^{(N-1)}+\overline{\mathcal{V}}_{a, 2}^{(N-1)}\right]\left(\psi^{[h, N-1]}\right)=-\tilde{\lambda}_{N-1}^{(\varepsilon)} \frac{Z_{N-2}^{2}}{Z_{N}} \bar{F}_{\lambda}^{[h, N-1]}\left(\psi^{[h, N-1]}, J\right)- \\
& -\tilde{z}_{N-1}^{(\varepsilon)} \frac{Z_{N-1}}{Z_{N}} \widehat{\psi}_{\mathbf{k}, \omega}^{[h, N-1]+} D_{\omega}(\mathbf{k}) \widehat{g}_{\omega}(\mathbf{k}) \widehat{J}_{\mathbf{k}, \omega}
\end{aligned}
$$

where we used the definition

$$
\bar{F}_{\lambda}\left(\psi^{[h, N-1]}, J\right)=\int \frac{d \mathbf{k}_{1} d \mathbf{k}_{2}}{(2 \pi)^{4}} \widehat{J}_{\mathbf{k}, \omega} \widehat{g}_{\omega}(\mathbf{k}) \widehat{\psi}_{\mathbf{k}_{2}, \omega}^{+} \widehat{\psi}_{\mathbf{k}+\mathbf{k}_{1}-\mathbf{k}_{2},-\omega}^{+} \widehat{\psi}_{\mathbf{k}_{1},-\omega}^{-}
$$

Let us consider now the terms contributing to $\overline{\mathcal{V}}_{b, i}^{(N-1)}$, that is those where $\widehat{\psi}_{\overline{\mathbf{k}}-\mathbf{p}, \omega}^{+}$is not contracted. Such terms can be analyzed exactly as in $\S 4.3$ of [BM4]; it turns out that

$$
\begin{aligned}
& \mathcal{L}\left[\overline{\mathcal{V}}_{b, 1}^{(N-1)}+\overline{\mathcal{V}}_{b, 2}^{(N-1)}\right]\left(\psi^{[h, N-1]}\right)=-\nu_{+, N-1} Z_{N-2} T_{-\varepsilon}\left(\psi^{[h, N-1]}, J\right)- \\
& -\nu_{-, N-1} Z_{N-2} T_{\varepsilon}\left(\psi^{[h, N-1]}, J\right),
\end{aligned}
$$


$\nu_{ \pm, N-1}$ being exactly the same constants appearing in (129).

The integration over subsequent scales is performed in a similar way; as described in more details in $\S 4.4$ of [BM4], it turns out that, if $j \geq h_{\mathbf{k}}$, the local part of the terms linear in $J$ has the form (coinciding with eq. (131) of [BM4] for $Z_{N}=1$ and $\omega=\varepsilon=-1$ ):

$$
\begin{aligned}
& \mathcal{L} \overline{\mathcal{V}}^{(j)}\left(\psi^{[h, j]}\right)=Z_{N} T_{1}^{(\varepsilon)}\left(\psi^{[h, j]}, J\right)-\nu_{+, j} Z_{j-1} T_{-\varepsilon}\left(\psi^{[h, j]}, J\right)- \\
& -\nu_{-, j} Z_{j-1} T_{\varepsilon}\left(\left(\psi^{[h, j]}, J\right)-\tilde{\lambda}_{j}^{(\varepsilon)} \frac{Z_{j-1}^{2}}{Z_{N}} \bar{F}_{\lambda}^{[h, j]}\left(\psi^{[h, j]}, J\right)-\right. \\
& -\sum_{i=j}^{N-1} \tilde{z}_{i}^{(\varepsilon)} \frac{Z_{i}}{Z_{N}} \widehat{\psi}_{\mathbf{k}, \omega}^{[h, j]+} D_{\omega}(\mathbf{k}) \widehat{g}_{\omega}(\mathbf{k}) \widehat{J}_{\mathbf{k}, \omega}
\end{aligned}
$$

If $j<h_{\mathbf{k}}, \mathcal{L} \overline{\mathcal{V}}^{(j)}$ has the same structure, but there is indeed no term with two external legs, since $\widehat{\psi}_{\mathbf{k}, \omega}^{[h, j]+}=0$; for a similar reason the term with four external legs is different from 0 only if $3 \gamma^{j-1}>\gamma^{h_{\mathbf{k}}}$. However, the constants $\tilde{\lambda}_{j}^{(\varepsilon)}$ and $\tilde{z}_{i}^{(\varepsilon)}$ are defined for any $j>h$ and their value is independent of $\mathbf{k}$. Note also that, as in the expansion of a normal Schwinger function, we do not localize the terms with four external legs, containing both a $J$ vertex and a $\varphi$ vertex.

It follows that we can write $\widetilde{G}_{\varepsilon, \omega}^{2, N, h}(\mathbf{k})$ as a sum of trees with two special endpoints, similar to those described in detail in $\S 4.5$ of [BM4]; they differ from those present in the expansion of the function $\widehat{G}_{\omega}^{2, N, h}(\mathbf{k})$, see $\S 2.5$, since one of the special endpoints corresponds to one of the addenda in (149), to be called of type $T, T_{+}, T_{-}, \widetilde{\lambda}^{(\varepsilon)}, \widetilde{z}^{(\varepsilon)}$. By construction the constants $\nu_{ \pm, j}$ coincide with those introduced in $\S 3.1$, hence they verify (134). Moreover, it was shown in $\S 4.6)$ of [BM4], by a fixed point argument, that, if $\bar{\lambda}_{h}$ is small enough, it is possible to choose $\alpha_{+, h, N}=c_{1} \lambda+O\left(\lambda^{2}\right)$ and $\alpha_{-, h, N}=c_{3}+O(\lambda)$ so that there exist two positive constant, $C$ and $\vartheta$, independent of $h$ and $N$, such that, if $h+1 \leq j \leq N-1$,

$$
\left|z_{i} \alpha_{\varepsilon, h, N}-\widetilde{z}_{i}^{(\varepsilon)}\right| \leq C \bar{\lambda}_{h} \gamma^{-\vartheta(N-i)}, \quad\left|\lambda_{i} \alpha_{\varepsilon, h, N}-\widetilde{\lambda}_{i}^{(\varepsilon)}\right| \leq C \bar{\lambda}_{h} \gamma^{-\vartheta(N-i)} .
$$

Then we can write

$$
\widetilde{G}_{\varepsilon, \omega}^{2, N, h}(\mathbf{k}) \stackrel{\text { def }}{=} A_{\varepsilon, \omega}^{\widetilde{\lambda}, N, h}(\mathbf{k})+A_{\varepsilon, \omega}^{\widetilde{z}, N, h}(\mathbf{k})+A_{\varepsilon, \omega}^{T_{ \pm}, N, h}(\mathbf{k})+A_{\varepsilon, \omega}^{T, N, h}(\mathbf{k}),
$$

where $A_{\varepsilon, \omega}^{\widetilde{\lambda}, N, h}, A_{\varepsilon, \omega}^{\widetilde{z}, N, h}, A_{\varepsilon, \omega}^{T_{ \pm}, N, h}$ and $A_{\varepsilon, \omega}^{T, N, h}$ contain respectively one endpoint of type $\widetilde{\lambda}^{(\varepsilon)}, \widetilde{z}^{(\varepsilon)}, T_{ \pm}, T$.

In order to bound $A_{\varepsilon, \omega}^{T, N, h}$, we repeat the analysis in $\S 4.8$ in [BM4]. It follows that it is bounded by an expression similar to the r.h.s. of (106), with the following differences. Given a tree $\tau$ contributing to $A_{\varepsilon, \omega}^{T, N, h}$, the dimensional bound differs from that of a tree contributing to $\widehat{G}_{\omega}^{2, N, h}(\mathbf{k})$ for the following reasons: 


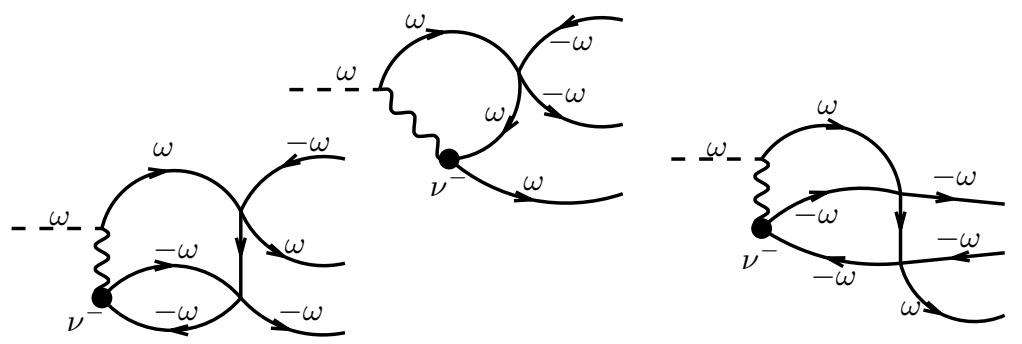

Figure 8: : Graphical representation of the leading terms contributing to $c_{1} \lambda$; other four graphs contributing to $c_{1} \lambda$, as well as the graph contributing to $c_{3}$, are vanishing in the limit of short tail $\left(\gamma_{0} \rightarrow 1\right)$ of the cutoff function (see definition (9)). The two graphs giving the 0 -th order expansion in $\lambda$ of $\alpha_{h, N}^{+}$cancell each other by symmetry.

(1) there is an extra factor $Z_{h_{\mathbf{k}}} / Z_{N}$, because one external propagator is substituted by the free one, $Z_{N}^{-1} \widehat{g}_{\omega}(\mathbf{k})$ (see the definition of $T_{1}^{(\varepsilon)}$ );

(2) since there is no external field renormalization for $T_{1}^{(\varepsilon)}$ (which is dimensionally equivalent to a term with four external fields), there is an extra factor $\left(Z_{N} / Z_{j_{T}}\right)^{2}$, if $j_{T}$ is the scale of the endpoint of type $T$;

(3) if at least one of fields in $T_{1}^{(\varepsilon)}$ ) is contracted on scale $h$, there is an extra factor $Z_{h} / Z_{N}$, because of the bound (119);

(4) because of (116), either $j_{T}=N+1$ or the root of $\tau$ has scale $h-1$.

Hence, $A_{\varepsilon, \omega}^{T, N, h}$ can be bounded by an expression equal to the r.h.s. of (106), multiplied by a factor $Z_{h} Z_{h_{\mathbf{k}}} / Z_{j_{T}}^{2} \leq \gamma^{C \lambda^{2}\left[\left(j_{T}-h\right)+\left|j_{T}-h_{\mathbf{k}}\right|\right]}$, which takes into account the items (1)-(3) above. This factor can be absorbed in the sum over the scale labels, since all vertices have an "effective" positive dimension (see remark before (107)). Then, by taking into account the remark in item (4) above, it is easy to show that

$$
\left|A_{\varepsilon, \omega}^{T, N, h}(\mathbf{k})\right| \leq C \frac{\gamma^{-h_{\mathbf{k}}}}{Z_{h_{\mathbf{k}}}}\left(\gamma^{-\left(N-h_{\mathbf{k}}\right) / 4}+\gamma^{-\left(h_{\mathbf{k}}-h\right) / 4}\right) .
$$

Let us now consider $A_{\varepsilon, \omega}^{T_{ \pm}, N, h}$. We still have some extra factors with respect to the bound (106), the same factor of item (1) above and a factor $\left(Z_{N} / Z_{j_{T}}\right)$, due to the partial field renormalization of $T_{ \pm}$; the product of these factors can be treated as before. We do not have anymore a condition like item (4) above, but we have to take into account that the running constant associated with the special vertex of type $T_{ \pm}$satisfies the bound (134). It follows that

$$
\left|A_{\varepsilon, \omega}^{T_{ \pm}, N, h}(\mathbf{k})\right| \leq C \frac{\gamma^{-h_{\mathbf{k}}}}{Z_{h_{\mathbf{k}}}} \gamma^{-\left(N-h_{\mathbf{k}}\right) / 4}
$$


Let us now consider $A_{\varepsilon, \omega}^{\widetilde{z}, N, h}$ and let us suppose that $|\mathbf{k}|=\gamma^{h_{\mathbf{k}}}$, so that $($ see $(60)$ and $(64)) \widehat{g}_{\omega}^{(j)}(\mathbf{k})=\left[D_{\omega}(\mathbf{k}) Z_{h_{\mathbf{k}}-1}\right]^{-1}$, if $j=h_{\mathbf{k}}$, while $\widehat{g}_{\omega}^{(j)}(\mathbf{k})=0$, if $j \neq h_{\mathbf{k}}$. This condition, which greatly simplifies the following discussion, is not really restrictive. In fact, since the external momentum $\mathbf{k}$ is fixed in this discussion, one could modify the definition (24) of the cutoff functions $f_{j}(\mathbf{p})$, by substituting it with $f_{j}\left(\mathbf{p} / p_{0}\right), p_{0}$ being a fixed positive number $\leq \gamma$, to be chosen so that $p_{0}^{-1}|\mathbf{k}|=\gamma^{h_{\mathbf{k}}}$, for some integer $h_{\mathbf{k}}$. Since our bounds would be clearly uniform in this new parameter and the removed cutoffs limit is independent of $\gamma$ (see $\S 2.5$ ), this procedure can not produce any trouble.

By using (149), we can write

$$
A_{\varepsilon, \omega}^{\widetilde{z}, N, h}(\mathbf{k})=A_{\varepsilon, \omega}^{1, \widetilde{z}, N, h}(\mathbf{k})-\frac{\widehat{g}_{\omega}(\mathbf{k})}{Z_{N}}\left[\frac{1}{Z_{h_{\mathbf{k}}-1}} \sum_{i=h_{\mathbf{k}}}^{N-1} \widetilde{z}_{i}^{(\varepsilon)} Z_{i}\right],
$$

where $A_{\varepsilon, \omega}^{1, \widetilde{z}, N, h}$ contains the contributions to $A_{\varepsilon, \omega}^{\widetilde{z}, N, h}$ coming from trees with at least one $\lambda$ endpoint. Since $Z_{j-1}=Z_{j}\left(1+z_{j}\right)$ and $Z_{N-1}=Z_{N}$,

$$
Z_{h_{\mathbf{k}}-1}-\sum_{j=h_{\mathbf{k}}}^{N-1} Z_{j} z_{j}=Z_{N}
$$

hence we can write

$$
\sum_{i=h_{\mathbf{k}}}^{N-1} \frac{\widetilde{z}_{i}^{(\varepsilon)} Z_{i}}{Z_{N}}=\sum_{i=h_{\mathbf{k}}}^{N-1}\left(\widetilde{z}_{i}^{(\varepsilon)}-z_{i} \alpha_{\varepsilon, h, N}\right) \frac{Z_{i}}{Z_{N}}+\alpha_{\varepsilon, h, N}\left(\frac{Z_{h_{\mathbf{k}}-1}}{Z_{N}}-1\right) .
$$

The first term in the r.h.s. of (156) can be written as

$$
\begin{aligned}
& \sum_{i=h_{\mathbf{k}}}^{N-1}\left(\widetilde{z}_{i}^{(\varepsilon)}-\alpha_{\varepsilon, h, N} z_{i}\right) \frac{Z_{i}}{Z_{N}}=\sum_{j=h}^{N-1}\left(\widetilde{z}_{j}^{(\varepsilon)}-\alpha_{\varepsilon, h, N} z_{j}\right) \frac{Z_{j}}{Z_{N}}-\sum_{j=h}^{h_{\mathbf{k}}-1}\left(\widetilde{z}_{j}^{(\varepsilon)}-\alpha_{\varepsilon, h, N} z_{j}\right) \frac{Z_{j}}{Z_{N}} \\
& \stackrel{\text { def }}{=}-\rho_{\varepsilon, h, N}+R_{\varepsilon}^{2, N, h}(\mathbf{k}),
\end{aligned}
$$

where $\rho_{\varepsilon, h, N}$ is independent of $\mathbf{k}$ and satisfies, by (150), the bound

$$
\left|\rho_{\varepsilon, h, N}\right| \leq C|\lambda| \sum_{j=h}^{N} \gamma^{-\left(\vartheta-c \bar{\lambda}_{h}^{2}\right)(N-j)} \leq C|\lambda|
$$

implying that there exists the limit $\rho_{\varepsilon}=\lim _{-h, N \rightarrow \infty} \rho_{\varepsilon, h, N}$. By an explicit computation one can show that $\rho_{+}=c_{2} \lambda+O\left(\lambda^{2}\right)$ and $\rho_{-}=c_{4}+O(\lambda)$, with $c_{2}$ and $c_{4}$ strictly positive constants. On the contrary, $R_{\varepsilon}^{2, N, h}(\mathbf{k})$ is vanishing for $-h, N \rightarrow \infty$; in fact

$$
\left|R_{\varepsilon}^{2, N, h}(\mathbf{k})\right| \leq C|\lambda| \sum_{j=h}^{h_{\mathbf{k}}} \gamma^{-\left(\vartheta-c \bar{\lambda}_{h}^{2}\right)(N-j)} \leq C|\lambda| \gamma^{-(\vartheta / 2)\left(N-h_{\mathbf{k}}\right)} .
$$




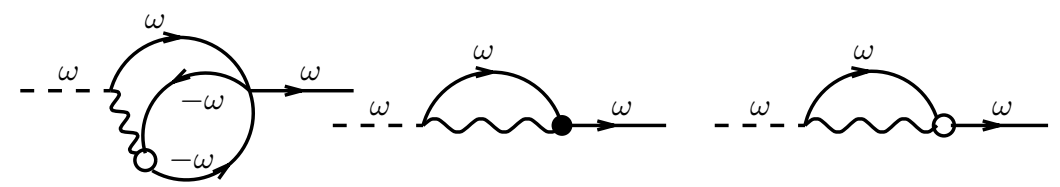

Figure 9: : The first two graphs are the graphical representation of $c_{2} \lambda$; the last is the graph for $c_{4}$.

By collecting all terms we get

$$
\begin{array}{r}
A_{\varepsilon, \omega}^{\widetilde{z}, N, h}(\mathbf{k})=A_{\varepsilon, \omega}^{1, \widetilde{z}, N, h}(\mathbf{k})-\alpha_{\varepsilon, N, h} \frac{\widehat{g}_{\omega}(\mathbf{k})}{Z_{N}}+ \\
+\left(\rho_{\varepsilon, N, h}+\alpha_{\varepsilon, N, h}\right) \widehat{g}_{\omega}^{\left(h_{\mathbf{k}}\right)}(\mathbf{k})-R_{\varepsilon}^{2, N, h}(\mathbf{k}) \widehat{g}_{\omega}^{\left(h_{\mathbf{k}}\right)}(\mathbf{k}),
\end{array}
$$

with $\left|R_{\varepsilon}^{2, N, h}(\mathbf{k}) \widehat{g}_{\omega}^{\left(h_{\mathbf{k}}\right)}(\mathbf{k})\right| \leq C|\lambda| \gamma^{-h_{\mathbf{k}}} Z_{h_{\mathbf{k}}}^{-1} \gamma^{-\vartheta\left(N-h_{\mathbf{k}}\right)}$.

We now consider $A^{1, \widetilde{z}, N, h}$ together to $A^{\widetilde{\lambda}, N, h}$. We proceed as in [BM3], formulas (161)-(165); to summarize, given a tree $\tau \in \mathcal{T}_{\lambda, n}, n \geq 1$, we can associate to it a tree $\tau^{\prime} \in \mathcal{T}_{z, n+1}$, substituting the endpoint $v^{*}$, on scale $j^{*}$, of type $\tilde{\lambda}$ with an endpoint of type $\lambda$, and linking the endpoint $v^{*}$ to an endpoint of type $\widetilde{z}$. If we define

$$
\widehat{G}_{\omega}^{2, N, h}(\mathbf{k}) \stackrel{\text { def }}{=} \widehat{g}_{\omega}^{\left(h_{\mathbf{k}}\right)}(\mathbf{k})+\sum_{j^{*}=h_{\mathbf{k}}}^{N} \lambda_{j^{*}} B_{\omega, j^{*}}^{2, N, h}(\mathbf{k}),
$$

then it is easy to check that

$$
\begin{aligned}
A_{\varepsilon, \omega}^{\widetilde{\lambda}, N, h}(\mathbf{k}) & =\frac{Z_{h_{\mathbf{k}}-1}}{Z_{N}} \sum_{j^{*}=h_{\mathbf{k}}}^{N-1} \widetilde{\lambda}_{j^{*}}^{(\varepsilon)} B_{\omega, j^{*}}^{2, N, h}(\mathbf{k}), \\
A_{\varepsilon, \omega}^{1, \widetilde{z}, N, h}(\mathbf{k}) & =-\frac{Z_{h_{\mathbf{k}}-1}}{Z_{N}} \sum_{j^{*}=h_{\mathbf{k}}}^{N-1} \lambda_{j^{*}}\left(\frac{\sum_{i=h_{\mathbf{k}}}^{N-1} \widetilde{z}_{i}^{(\varepsilon)} Z_{i}}{Z_{h_{\mathbf{k}}-1}}\right) B_{\omega, j^{*}}^{2, N, h}(\mathbf{k}) .
\end{aligned}
$$

Using (155) and the definitions of $\rho_{\varepsilon, h, N}$ and $R_{\varepsilon}^{2, N, h}(\mathbf{k})$, we get:

$$
\begin{aligned}
& \tilde{\lambda}_{j^{*}}^{(\varepsilon)}-\lambda_{j^{*}} \frac{\sum_{j=h_{\mathbf{k}}}^{N-1} \widetilde{z}_{j}^{(\varepsilon)} Z_{j}}{Z_{h_{\mathbf{k}}-1}}=\left(\widetilde{\lambda}_{j^{*}}^{(\varepsilon)}-\alpha_{\varepsilon} \lambda_{j^{*}}\right)+ \\
& +\lambda_{j^{*}} \frac{Z_{N}}{Z_{h_{\mathbf{k}}-1}}\left[\alpha_{\varepsilon, h, N}+\rho_{\varepsilon, h, N}-R_{\varepsilon}^{2, N, h}(\mathbf{k})\right] .
\end{aligned}
$$

By the usual arguments, one can see that $\left|B_{\omega, j^{*}}^{2, N, h}(\mathbf{k})\right| \leq C \gamma^{-h_{\mathbf{k}}} Z_{h_{\mathbf{k}}}^{-1}\left(\gamma^{-\vartheta\left|j^{*}-h_{\mathbf{k}}\right|}\right)$; hence, by summing the two equations in (162), we get:

$$
A_{\varepsilon, \omega}^{\widetilde{\lambda}, N, h}(\mathbf{k})+A_{\varepsilon, \omega}^{1, \widetilde{z}, N, h}(\mathbf{k})=\left(\alpha_{\varepsilon, h, N}+\rho_{\varepsilon, h, N}\right)\left[\widehat{G}_{\omega}^{2, N, h}(\mathbf{k})-\widehat{g}_{\omega}^{\left(h_{\mathbf{k}}\right)}(\mathbf{k})\right]+R_{\varepsilon}^{3, N, h}(\mathbf{k}),
$$


where

$R_{\varepsilon}^{3, N, h}(\mathbf{k}) \stackrel{\text { def }}{=} \frac{Z_{h_{\mathbf{k}}-1}}{Z_{N}} \sum_{j^{*}=h_{\mathbf{k}}}^{N} B_{\omega, j^{*}}^{2, N, h}(\mathbf{k})\left[\left(\tilde{\lambda}_{j^{*}}^{(\varepsilon)}-\alpha_{\varepsilon, h, N} \lambda_{j^{*}}\right)-\frac{Z_{N}}{Z_{h_{\mathbf{k}}-1}} \lambda_{j^{*}} R_{\varepsilon}^{2, N, h}(\mathbf{k})\right]$

is bounded by $C|\lambda| \gamma^{-h_{\mathbf{k}}} Z_{h_{\mathbf{k}}}^{-1} \gamma^{-(\vartheta / 2)\left(N-h_{\mathbf{k}}\right)}$.

Finally, the summation of all terms in the r.h.s. of (151) gives

$$
\widetilde{G}_{\varepsilon, \omega}^{2, N, h}(\mathbf{k})=-\alpha_{\varepsilon, h, N} \frac{g_{\omega}(\mathbf{k})}{Z_{N}}+\left(\alpha_{\varepsilon, h, N}+\rho_{\varepsilon, h, N}\right) G_{\omega}^{2, N, h}(\mathbf{k})+R_{\varepsilon}^{4, N, h}(\mathbf{k}),
$$

with

$$
\left|R_{\varepsilon}^{4, N, h}(\mathbf{k})\right| \leq C|\lambda| \frac{\gamma^{-h_{\mathbf{k}}}}{Z_{h_{\mathbf{k}}}}\left(\gamma^{-(\vartheta / 2)\left(h_{\mathbf{k}}-h\right)}+\gamma^{-(\vartheta / 2)\left(N-h_{\mathbf{k}}\right)}\right) .
$$

This ends the proof of Theorem 3 .

\subsection{Proof of Corollary 1}

If we insert the identity (166) in the r.h.s. of (31) and we take the limit $h \rightarrow-\infty$, we get

$$
\widehat{G}_{\omega}^{2, N}(\mathbf{k}) D_{\omega}(\mathbf{k})=\frac{B_{N}}{Z_{N}}-b_{N} \int \frac{d \mathbf{p}}{(2 \pi)^{2}} \bar{\chi}_{N}(\mathbf{p}) \frac{\widehat{G}_{\omega}^{2, N}(\mathbf{k}-\mathbf{p})}{D_{-\omega}(\mathbf{p})}+H_{N, \omega}(\mathbf{k}),
$$

where $\bar{\chi}_{N}(\mathbf{p})$ is the function appearing in (30), $B_{N} \equiv\left(1-\lambda_{N} \sum_{\varepsilon} A_{\varepsilon, N} a_{\varepsilon, N}\right)[1-$ $\left.\lambda_{N} \sum_{\varepsilon} A_{\varepsilon, N}\left(a_{\varepsilon, N}+\rho_{\varepsilon, N}\right)\right]^{-1}, b_{N} \equiv \lambda_{N} A_{+, N}\left[1-\lambda_{N} \sum_{\varepsilon} A_{\varepsilon, N}\left(a_{\varepsilon, N}+\rho_{\varepsilon, N}\right)\right]^{-1}$ and $H_{N, \omega}(\mathbf{k})$ is a function satisfying the bound

$$
\left|H_{N, \omega}(\mathbf{k})\right| \leq C|\lambda| Z_{h_{\mathbf{k}}}^{-1} \gamma^{-(\vartheta / 2)\left(N-h_{\mathbf{k}}\right)} .
$$

On the other hand, by (108), there is a function $f(\lambda)$, independent of $\mathbf{k}$, such that

$$
\widehat{G}_{\omega}^{2, N}(\mathbf{k})=\frac{|\mathbf{k}|^{\eta_{z}}}{D_{\omega}(\mathbf{k})} F_{N}(\mathbf{k}) \quad, \quad F_{N}(\mathbf{k})=f(\lambda)+O\left(\gamma^{-N}|\mathbf{k}|\right)^{\vartheta}
$$

hence, we can rewrite (168) as

$$
|\mathbf{k}|^{\eta_{z}} F_{N}(\mathbf{k})=\frac{B_{N}}{Z_{N}}+b_{N} \int \frac{d \mathbf{p}}{(2 \pi)^{2}} \bar{\chi}_{N}(\mathbf{p}+\mathbf{k})|\mathbf{p}|^{\eta_{z}} \frac{F_{N}(\mathbf{p})}{D_{-\omega}(\mathbf{p}+\mathbf{k}) D_{\omega}(\mathbf{p})}+H_{N, \omega}(\mathbf{k})
$$

and, subtracting the equation with $\mathbf{k}=0$, we obtain

$$
\begin{aligned}
& |\mathbf{k}|^{\eta_{z}} F_{N}(\mathbf{k})=b_{N} \int \frac{d \mathbf{p}}{(2 \pi)^{2}} \frac{|\mathbf{p}|^{\eta_{z}}}{D_{\omega}(\mathbf{p})} F_{N}(\mathbf{p})\left[\frac{\bar{\chi}_{N}(\mathbf{k}+\mathbf{p})}{D_{-\omega}(\mathbf{k}+\mathbf{p})}-\frac{\bar{\chi}_{N}(\mathbf{p})}{D_{-\omega}(\mathbf{p})}\right]+ \\
& +H_{N ; \omega}(\mathbf{k})-H_{N, \omega}(0)
\end{aligned}
$$


The integral can be written as the sum of two terms

$$
\begin{aligned}
& \int \frac{d \mathbf{p}}{(2 \pi)^{2}} \frac{|\mathbf{p}|^{\eta_{z}}}{|\mathbf{p}|^{2}} F_{N}(\mathbf{p}) \bar{\chi}_{N}(\mathbf{k}+\mathbf{p}) \frac{D_{-\omega}(\mathbf{k})}{D_{-\omega}(\mathbf{k}+\mathbf{p})}- \\
& -\int \frac{d \mathbf{p}}{(2 \pi)^{2}} \frac{|\mathbf{p}|^{\eta_{z}}}{|\mathbf{p}|^{2}} F_{N}(\mathbf{p})\left[\bar{\chi}_{N}(\mathbf{k}+\mathbf{p})-\bar{\chi}_{N}(\mathbf{p})\right]
\end{aligned}
$$

and the second addend is vanishing in the $N \rightarrow \infty$ limit, as it can be written as

$$
\gamma^{\eta_{z} N} \int \frac{d \mathbf{p}}{(2 \pi)^{2}} \frac{|\mathbf{p}|^{\eta_{z}}}{|\mathbf{p}|^{2}} F_{N}(\mathbf{p})\left[\bar{\chi}_{0}\left(\gamma^{-N} \mathbf{k}+\mathbf{p}\right)-\bar{\chi}_{0}(\mathbf{p})\right]
$$

and $\bar{\chi}_{0}\left(\gamma^{-N} \mathbf{k}+\mathbf{p}\right)-\bar{\chi}_{0}(\mathbf{p})$ is $O\left(\gamma^{-N}|\mathbf{k}|\right)$ and with compact support. On the other hand, by (170), the integral we obtain, if we substitute $F_{N}(\mathbf{p})$ with $f(\lambda)$, is vanishing as $N \rightarrow \infty$. Hence, in the limit $N \rightarrow \infty$ we get the identity:

$$
|\mathbf{k}|^{\eta_{z}}=b_{\infty} \int \frac{d \mathbf{p}}{(2 \pi)^{2}} \frac{|\mathbf{p}|^{\eta_{z}}}{|\mathbf{p}|^{2}} \frac{D_{-\omega}(\mathbf{k})}{D_{-\omega}(\mathbf{k}+\mathbf{p})}=\frac{b_{\infty}}{2 \pi} \int_{0}^{\infty} \frac{d \rho}{\rho^{1-\eta_{z}}} \int_{0}^{2 \pi} \frac{d \vartheta}{2 \pi} \frac{|\mathbf{k}|}{|\mathbf{k}|+\rho e^{i \vartheta}}
$$

that is

$$
1=\frac{b_{\infty}}{2 \pi} \int_{0}^{\infty} \frac{d \rho}{\rho^{1-\eta_{z}}} \int_{0}^{2 \pi} \frac{d \vartheta}{2 \pi} \frac{1}{1+\rho e^{i \vartheta}}=\frac{b_{\infty}}{2 \pi} \int_{0}^{1} \frac{d \rho}{\rho^{1-\eta_{z}}}=\frac{b_{\infty}}{2 \pi \eta_{z}}
$$

which proves $(36)$.

\subsection{Proof of Theorem 5}

The Beta function equations for the running coupling or renormalization constants are

$$
\begin{aligned}
\lambda_{j-1} & =\lambda_{j}+\beta_{\lambda}^{j}\left(\lambda_{j}, \ldots, \lambda_{N}\right), \\
\frac{Z_{j-1}}{Z_{j}} & =1+\beta_{z}^{(j)}\left(\lambda_{j}, . ., \lambda_{N}\right), \\
\frac{Z_{j-1}^{(2)}}{Z_{j}^{(2)}} & =1+\beta_{z_{2}}^{(j)}\left(\lambda_{j}, \ldots, \lambda_{N}\right), \\
\frac{\mu_{j-1}}{\mu_{j}} & =1+\sum_{k \geq j} \frac{\mu_{k}}{\mu_{j}} \beta_{\mu}^{(j, k)}\left(\lambda_{j}, . ., \lambda_{N}\right),
\end{aligned}
$$

with $\beta_{z}^{(j)}, \beta_{z_{2}}^{(j)}, \beta_{\mu}^{(j, k)}$ independent from $\mu$ and, if $a_{\mu}, a_{z}, a_{z_{2}}$ are suitable positive constants,

$$
\begin{aligned}
\beta_{\mu}^{(j, k)}\left(\lambda_{j}, . . \lambda_{j}\right) & =a_{\mu} \lambda_{j} \delta_{j, k}+O\left(\bar{\lambda}_{j}^{2}\right), \\
\beta_{z}^{(j)}\left(\lambda_{j}, . ., \lambda_{j}\right) & =a_{z} \lambda_{j}^{2}+O\left(\bar{\lambda}_{j}^{4}\right) \\
\beta_{z_{2}}^{(j)}\left(\lambda_{j}, . ., \lambda_{j}\right) & =a_{z_{2}} \lambda_{j}^{2}+O\left(\bar{\lambda}_{j}^{4}\right) .
\end{aligned}
$$


Moreover, these functions do not depend directly of $Z_{N}$, but only depend on the ratios $Z_{j-1} / Z_{j}, j \leq N$; hence the value of $\lambda_{j}$ is a function of $\lambda_{N}=\lambda$ and the number of RG steps needed to reach scale $j$ starting from scale $N$. It follows that, if we call $\widehat{\lambda}_{j}, j \leq 0$, the constants we get for $N=0$, then, for any $N>0$ and $j \leq N, \lambda_{j}=\widehat{\lambda}_{j-N}$. The problem with $N=0$ was studied in detail in [BM4], where it has been proved (see Theorem 2 of that paper) that there exist constants $c_{1}, \varepsilon_{1}$ (independent of $N, h$ ), such that, if $|\lambda| \leq \varepsilon_{1}$, then $\left|\lambda_{j}\right| \leq c_{1} \varepsilon_{1}$ for any $j$. The proof of this statement is based on the analogue of SDe equation (31) for the four point function; if the momenta are calculated at the infrared cut-off scale $\gamma^{j}$, a relation is obtained between $\lambda_{j}$ and $\lambda$ implying that $\lambda_{j}=\lambda+O\left(\lambda^{2}\right)$. This properties implies, see (3.48) of [BM3], that

$$
\left|\beta_{\lambda}^{j}\left(\lambda_{j}, \ldots, \lambda_{j}\right)\right| \leq C\left|\lambda_{j}\right|^{2} \gamma^{-(N-j) / 4}
$$

From (177) and (193) one gets immediately, see $\S 4.10$ of [BM1], the bound (70) with $\lambda_{-\infty}(\lambda)=\lambda+O\left(\lambda^{2}\right)$ together with $\left|\log _{\gamma}\left(Z_{j-1} / Z_{j}\right)-\eta_{z}\right| \leq C \lambda^{2} \gamma^{-(N-j) / 4}$, $\left|\log _{\gamma}\left(\mu_{j-1} / \mu_{j}\right)-\eta_{\mu}\right| \leq C|\lambda| \gamma^{-(N-j) / 4}$; finally by the WTi (26) with momenta calculated at the infrared cut-off scale $\gamma^{j}$ one gets, see [BM2], $\left|Z_{j}^{(2)} / Z_{j}-1\right| \leq$ $C|\lambda|$.

\section{$5 \quad$ Lattice Wilson fermions}

\subsection{Integration of the doubled fermions}

In order to prove Theorem 4, we have to compare the Schwinger functions of the continuum model with ultraviolet cutoff scale $N$ with those of the lattice model (37) with $a=\pi /\left(4 \gamma^{N+1}\right)$. In this model the momentum $\mathbf{k}$ belongs to the two-dimensional torus $\mathcal{D}_{a}$ of size $2 \pi / a$ and we shall denote by $\left|\mathbf{k}-\mathbf{k}^{\prime}\right|$ the corresponding distance.

To begin with, we define $\bar{f}(\mathbf{k})$ so that

$$
C_{N}^{-1}(\mathbf{k})+\bar{f}(\mathbf{k})=1,
$$

where $C_{N}^{-1}(\mathbf{k})=\sum_{j=-\infty}^{N} f_{j}(\mathbf{k})$, with $f_{j}(\mathbf{k})$ as in $(10)$; since $C_{N}^{-1}(\mathbf{k})=0$ for $|\mathbf{k}| \geq \gamma^{N+1}=\pi /(4 a)$, the support of the function $\bar{f}(\mathbf{k})$ is given by the set $\{\mathbf{k}:|\mathbf{k}-\pi / a| \leq 3 \pi / 4 a\}$. Therefore, it is possible to decompose the propagator $\widehat{r}_{\omega, \omega^{\prime}}(\mathbf{k})$, defined in $(38)$, as the sum of $\widehat{r}_{\omega, \omega^{\prime}}^{(\leq N)}(\mathbf{k})=C_{N}^{-1}(\mathbf{k}) \widehat{r}_{\omega, \omega^{\prime}}(\mathbf{k})$ and $\widehat{r}_{\omega, \omega^{\prime}}^{(N+1)}(\mathbf{k})=\bar{f}(\mathbf{k}) \widehat{r}_{\omega, \omega^{\prime}}(\mathbf{k})$. With this decomposition we associate the following decomposition of the measure (37)

$$
P_{Z_{a}}(d \psi)=P_{Z_{a}}\left(d \psi^{(\leq N)}\right) P_{Z_{a}}\left(d \psi^{(N+1)}\right) .
$$

Note that the second integration has a "very massive" propagator; in fact, since the function $\bar{f}(\mathbf{k})$ is a Gevrais function of class 2, with a compact 
support of size $a^{-2}$, and $\left[1-\cos \left(k_{0} a\right)+1-\cos (k a)\right] / a \geq \tilde{C} a^{-1}=C \gamma^{N}$ on its support, it is easy to show that

$$
\left|r_{\omega, \omega^{\prime}}^{(N+1)}(\mathbf{x})\right| \leq C \gamma^{N} e^{-c \sqrt{\gamma^{N}|\mathbf{x}|}} .
$$

The integration of $\mathcal{W}(\varphi, J)$ is performed in a way very similar to the one presented in $\S 2$, except for the first step, made with $Z_{N+1}=Z_{a}, \lambda_{N+1}=\lambda_{a}$, $\nu_{N+1}=\nu_{a}, \mu_{N+1}(\mathbf{k})=\mu_{a}(\mathbf{k})$. We define all localization operators as in $\S 2$, except $\mathcal{L}_{1}$, which is defined as

$$
\mathcal{L}_{1} \widehat{W}_{2, \omega, \omega^{\prime}}^{(h)}(\mathbf{k})=\frac{1}{4} \sum_{\eta, \eta^{\prime}= \pm 1} \widehat{W}_{2, \omega, \omega^{\prime}}^{(h)}\left(\overline{\mathbf{k}}_{\eta \eta^{\prime}}\right)\left[\eta \frac{\sin k_{0} a}{\sin \frac{\pi a}{L}}+\eta^{\prime} \frac{\sin k a}{\sin \frac{\pi a}{L}}\right],
$$

in order to take into account the lattice structure of the space coordinates; hence the localization procedure is essentially unchanged. However, the presence in the interaction of the term proportional to $\nu_{N+1}$ has the effect (see below) that in the effective potential a new type of vertex will appear (which we shall call $\nu$ vertex); this new vertex changes the symmetry properties of the functions $\widehat{W}_{2, \omega, \omega^{\prime}}^{(j)}$, so that, in particular, $\mathcal{P}_{0} \widehat{W}_{2, \omega,-\omega}^{(j)} \neq 0$.

To be more precise, we note that $\widehat{W}_{2, \omega, \omega}^{(j)}$ is given by the sum of graphs with

1. either an even number of $\nu$ vertices, an even number of non diagonal propagators and an odd number of diagonal propagators;

2. or an odd number of $\nu$ vertices, an odd number of non diagonal propagators and an odd number of diagonal propagators.

Moreover $\widehat{W}_{2, \omega,-\omega}^{(j)}$ is given by the sum of graphs with

3. either an even number of $\nu$ vertices, an odd number of non diagonal propagators and an even number of diagonal propagators;

4. or an odd number of $\nu$ vertices, an even number of non diagonal propagators and an even number of diagonal propagators.

As the diagonal propagators are odd in the exchange $\mathbf{k} \rightarrow-\mathbf{k}$ while the non diagonal ones are even, we get $\mathcal{L}_{0} \mathcal{P}_{0} \widehat{W}_{2, \omega, \omega}^{(j)}=\mathcal{L}_{0} \mathcal{P}_{1} \widehat{W}_{2, \omega, \omega}^{(j)}=0$ and $\mathcal{L}_{1} \mathcal{P}_{0} \widehat{W}_{2, \omega,-\omega}^{(j)}=0$. Then

$$
\mathcal{L} \widehat{W}_{2, \omega, \omega}^{(j)}=\mathcal{L}_{1} \mathcal{P}_{0} \widehat{W}_{2, \omega, \omega}^{(j)}, \quad \mathcal{L} \widehat{W}_{2, \omega,-\omega}^{(j)}=\mathcal{L}_{0} \mathcal{P}_{0} \widehat{W}_{2, \omega,-\omega}^{(j)}+\mathcal{L}_{0} \mathcal{P}_{1} \widehat{W}_{2, \omega,-\omega}^{(j)} .
$$

This implies that we can write

$$
\mathcal{L} \mathcal{V}^{(j)}\left(\psi^{[h, j]}\right)=z_{j} F_{\zeta}^{[h, j]}+\left(s_{j}+\gamma^{j} n_{j}\right) F_{\sigma}^{[h, j]}+l_{j} F_{\lambda}^{[h, j]},
$$

where $\gamma^{j} n_{j}=\mathcal{L}_{0} \mathcal{P}_{0} \widehat{W}_{2, \omega,-\omega}^{(j)}$, while $s_{j}=\mathcal{L}_{0} \mathcal{P}_{1} \widehat{W}_{2, \omega,-\omega}^{(j)}$, as in (50)-(51). 
The renormalization of the free measure is done exactly as in $\S 2$, see (60), that is we do not put the term proportional to $n_{j}$ in the free measure, but we define a new running coupling constant $\nu_{j}=n_{j}\left(Z_{j} / Z_{j-1}\right)$. It follows that the rescaled potential $\widehat{\mathcal{V}}^{(j)}\left(\psi^{[h, j]}\right)$ differs from that of $(67)$ because its local part contains the term $\gamma^{j} \nu_{j} F_{\sigma}^{[h, j]}$.

For $j \leq N$, the renormalized measure takes the form:

$$
\widehat{r}_{\omega, \omega^{\prime}}^{(j)}(\mathbf{k}) \stackrel{\text { def }}{=} \frac{\tilde{f}_{j}(\mathbf{k})}{e_{+}(\mathbf{k}) e_{-}(\mathbf{k})-\mu_{j}^{2}(\mathbf{k})}\left(\begin{array}{cc}
e_{-}(\mathbf{k}) & -\tilde{\mu}_{j}(\mathbf{k}) \\
-\tilde{\mu}_{j}(\mathbf{k}) & e_{+}(\mathbf{k})
\end{array}\right)_{\omega, \omega^{\prime}}
$$

with $\tilde{\mu}_{j}(\mathbf{k})=\widehat{\mu}_{j}(\mathbf{k})+\left[Z_{N} / Z_{j}(\mathbf{k})\right]\left[1-\cos \left(k_{0} a\right)+1-\cos (k a)\right] / a, \widehat{\mu}_{j}(\mathbf{k})$ being a function equal to $\mu$ for $j=N$, which satisfies the same recursion relation as $\tilde{\mu}_{j}(\mathbf{k})$ in $(60)$.

It is convenient to split the propagator (187) as

$$
r_{\omega, \omega^{\prime}}^{(j)}(\mathbf{x}, \mathbf{y})=g_{\omega, \omega^{\prime}}^{(j)}(\mathbf{x}, \mathbf{y})+g_{\omega, \omega^{\prime}}^{R,(j)}(\mathbf{x}, \mathbf{y})
$$

where $g_{\omega, \omega^{\prime}}^{(j)}$ is obtained from $r_{\omega, \omega^{\prime}}^{(j)}$ by substituting $\tilde{\mu}_{j}(\mathbf{k})$ with $\widehat{\mu}_{j}(\mathbf{k})$ (hence it has the same form as the propagator of (43)). We shall prove below that the flow of the running couplings and the free measure can be controlled as in $\S 2$, if the value of $\nu_{a}$ is suitable chosen. This implies that there is $h^{*}$, satisfying a bound like (73), such that, as far as $h>h^{*},\left|\tilde{\mu}_{j}(\mathbf{k})\right| \leq \gamma^{j}$, so that

$$
\left|g_{\omega, \omega^{\prime}}^{R,(j)}(\mathbf{x}, \mathbf{y})\right| \leq C \gamma^{-(N-j)} \gamma^{j} e^{-c \sqrt{\gamma^{j}|\mathbf{x}-\mathbf{y}|}} .
$$

The flow equation for $\lambda_{j}$ can be written, for $j \leq N+1$, as

$$
\begin{aligned}
\lambda_{j-1}=\quad & \lambda_{j}+\beta_{\lambda}^{j}\left(\lambda_{N}, \ldots, \lambda_{j}\right)+r_{\lambda}^{j}\left(\lambda_{a}, \lambda_{N}, \ldots, \lambda_{j}\right) \\
& +\sum_{k \geq j} \nu_{k} \tilde{\beta}_{\lambda}^{j, k}\left(\lambda_{a}, \nu_{a}, \lambda_{N}, \nu_{N}, \ldots, \lambda_{j}, \nu_{j}\right),
\end{aligned}
$$

where the functions in the r.h.s. can be represented as sums over trees similar to those of (69); in particular, we have included the sum over all trees with at least one $\nu$-endpoint in the last term in the r.h.s. of (191) and we have split the sum of all trees with no $\nu$-endpoints as $\beta_{\lambda}^{j}+r_{\lambda}^{j}$, where $\beta_{\lambda}^{j}$ contains the trees with propagator $g_{\omega, \omega^{\prime}}^{(j)}$ (the decomposition (188) is used), while all other terms are included in $r_{\lambda}^{j}$. The fact that the contribution of a single tree satisfies a bound similar to that of (77), with $d_{v}>0$ for any $v$, easily implies that, if $\left|\nu_{j}\right| \leq C\left|\lambda_{a}\right|$ for any $j$,

$$
\left|\tilde{\beta}_{\lambda}^{j, k}\right| \leq C \bar{\lambda}_{j} \gamma^{-(k-j) / 4} \quad, \quad\left|r_{\lambda}^{j}\right| \leq C \bar{\lambda}_{j}^{2} \gamma^{-(N-j) / 4} .
$$

Note also that (193) still holds, as the only difference comes from the fact that in the continuum model the delta function of conservation of momenta is 
$L^{2} \delta_{k, 0} \delta_{k_{0}, 0}$, while in the lattice model is $L^{2} \sum_{n, m \in Z^{2}} \delta_{k, 2 \pi n / a} \delta_{k_{0}, 2 \pi m / a}$. However, the difference between the two delta functions has no effect on the local part $\mathcal{L} \mathcal{V}^{N}$, because of the compact support of $\psi^{\leq N}$ and only slightly affects the non local terms. To see that, let us consider a particular tree $\tau$ and a vertex $v \in \tau$ of scale $h_{v}$ with $2 n$ external fields of space momenta $\mathbf{k}_{i}$; the conservation of momentum implies that $\sum_{i} \varepsilon_{i} \mathbf{k}_{i}=\mathbf{m} \frac{2 \pi}{a}$, with $m$ an arbitrary integer. On the other hand, $\mathbf{k}_{i}$ is of order $\gamma^{h_{v}}$ for any $i$, hence $m$ can be different from 0 only if $n$ is of order $\gamma^{N-h_{v}}$. Since the number of endpoints following a vertex with $2 n$ external fields is greater or equal to $n-1$ and there is a small factor (of order $\bar{\lambda}_{j}$ ) associated with each endpoint, we get an improvement, in the bound of the terms with $|m|>0$, with respect to the others, of a factor $\exp \left(-C \gamma^{N-h_{v}}\right)$. Hence, by using the remark preceding (192), it is easy to show that the difference between the two beta functions is of order $\bar{\lambda}_{j}^{2} \gamma^{-(N-j) / 4}$.

Lemma 1 For any given $\lambda_{N+1}$ small enough, it is always possible to fix $\nu_{N+1}$ so that, for any $j \leq N+1$,

$$
\left|\nu_{j}\right| \leq C\left|\lambda_{a}\right| \gamma^{-(N-j) / 8}, \quad\left|\lambda_{j}-\lambda_{a}\right| \leq C \lambda_{a}^{2} .
$$

Proof. We consider the Banach space $\mathcal{M}_{\xi}$ of sequences $\underline{\nu}=\left\{\nu_{j}\right\}_{j \leq N+1}$ such that

$$
\|\underline{\nu}\|_{\xi}=\sup _{j \leq N+1} \gamma^{(N-j) / 8}\left|\nu_{j}\right| \leq \xi\left|\lambda_{a}\right|,
$$

with $\xi$ to be fixed later. From (191), (192) and (193) it follows, see $\S 4$ of [BM1] or Appendix 5 of [GiM] for details, that there exists $\varepsilon_{0}$ such that, if both $\left|\lambda_{a}\right|$ and $\xi\left|\lambda_{a}\right|$ are smaller than $\varepsilon_{0}$, then, for any $\underline{\nu}, \underline{\nu^{\prime}} \in \mathcal{M}_{\xi}$,

$$
\left|\lambda_{j}(\underline{\nu})-\lambda_{a}\right| \leq C \lambda_{a}^{2} \quad, \quad\left|\lambda_{j}(\underline{\nu})-\lambda_{j}\left(\underline{\nu}^{\prime}\right)\right| \leq C\left|\lambda_{a}\right|\left|\underline{\nu}-\underline{\nu^{\prime}}\right|_{\xi} .
$$

We want to show that it is possible to choose $\nu_{N+1}$ so that $\underline{\nu} \in \mathcal{M}_{\xi}$. Note that $\underline{\nu}$ verifies by construction the equation

$$
\nu_{j-1}=\gamma \nu_{j}+\beta_{\nu}^{(j)}\left(\lambda_{a}, \nu_{a} ; \lambda_{N}, \nu_{N} ; \ldots ; \lambda_{j}, \nu_{j}\right)
$$

and that, if $\underline{\nu} \in \mathcal{M}_{\xi}, \lim _{j \rightarrow-\infty} \nu_{j}=0$; by some simple algebra, this implies that

$$
\nu_{j}=-\sum_{k \leq j} \gamma^{k-j-1} \beta_{\nu}^{(k)}\left(\lambda_{a}, \nu_{a} ; \lambda_{N}, \nu_{N} ; \ldots ; \lambda_{j}, \nu_{j}\right) .
$$

Hence, we look for a fixed point of the operator $\mathbf{T}: \mathcal{M}_{\xi} \rightarrow \mathcal{M}_{\xi}$ defined as

$$
\mathbf{T}(\underline{\nu}){ }_{j} \stackrel{\text { def }}{=}-\sum_{k \leq j} \gamma^{k-j-1} \beta_{\nu}^{(k)}\left(\lambda_{a}, \nu_{a}, \lambda_{N}(\underline{\nu}), \nu_{N}, . ., \lambda_{j}(\underline{\nu}), \nu_{j}\right) .
$$


Note that

$$
\begin{aligned}
& \beta_{\nu}^{(j)}\left(\lambda_{a}, \nu_{a}, \lambda_{N}, \nu_{N}, \ldots, \lambda_{j}, \nu_{j}\right)=\beta_{\nu}^{(1, j)}\left(\lambda_{N}, \ldots, \lambda_{j}\right)+ \\
& +\sum_{k \geq j} \nu_{k} \tilde{\beta}_{\nu}^{(j, k)}\left(\lambda_{a}, \nu_{a}, \lambda_{N}, \nu_{N}, \ldots, \lambda_{j}, \nu_{j}\right)
\end{aligned}
$$

where $\beta_{\nu}^{(j, 1)}$ is a sum over trees with no endpoints of type $\nu$ and no endpoints of scale $N+1$. By using the decomposition (188), the parity properties of $g_{\omega, \omega^{\prime}}^{(j)}(\mathbf{x}, \mathbf{y})$ and the remark preceding (192), we get the bounds

$$
\left|\beta_{\nu}^{(1, j)}\right| \leq C\left|\lambda_{a}\right| \gamma^{-(N-j) / 4} \quad, \quad\left|\tilde{\beta}_{\nu}^{(j, k)}\right| \leq C\left|\lambda_{a}\right| \gamma^{-(k-j) / 4},
$$

which implies that

$$
\left|\mathbf{T}(\underline{\nu})_{j}\right| \leq \sum_{k \leq j} C\left|\lambda_{a}\right| \gamma^{-(j-k)} \gamma^{-(N-k) / 8} \leq c_{0}\left|\lambda_{a}\right| \gamma^{-(N-j) / 8} .
$$

Hence the operator $\mathbf{T}: \mathcal{M}_{\xi} \rightarrow \mathcal{M}_{\xi}$ leaves $\mathcal{M}_{\xi}$ invariant, if $\xi \geq c_{0}$ and $\lambda_{a}$ is sufficiently small, and it is also a contraction since $\left|\mathbf{T}(\underline{\nu})_{j}-\mathbf{T}\left(\underline{\nu^{\prime}}\right)_{j}\right| \leq$ $C\left|\lambda_{a}\right||| \nu-\nu^{\prime}||_{\xi}$. It follows that there is a unique fixed point in $\mathcal{M}_{\xi}$, satisfying the flow equation (196).

An important consequence of the bound (193) is that, if we construct as in $\S 2$ the Schwinger functions, by imposing the normalization conditions (71), we get, as $N \rightarrow \infty$, exactly the same expansion in terms of trees, containing only $\lambda$ endpoints with a fixed coupling constant $\tilde{\lambda}_{-\infty}\left(\lambda_{a}\right)=$ $\lim _{j \rightarrow-\infty} \lambda_{j}$; in fact, the trees containing at least one $\nu$ vertex vanish in this limit.

By a fixed point argument, one can show that we can fix $\lambda_{a}$ so that $\tilde{\lambda}_{-\infty}\left(\lambda_{a}\right)$ has the same value as $\lambda_{-\infty}(\lambda)$ in the continuum model; this remark completes the proof of Theorem 4 .

\section{A Osterwalder-Schrader axioms}

Osterwalder-Schrader axioms were partially stated in [OS1] and completed in [OS2] by the "linear growth property". We show here that they are satisfied by the Schwinger functions of our model.

\section{A.1 Linear growth condition and Clustering}

In order to verify the linear growth property, see the bound (4.1) of [OS2], for $s=3$, let us consider the space $\mathcal{S}_{0}\left(\mathbb{R}^{2 k}\right)$ of the test functions such that, for any $m \in \mathbb{N}$,

$$
\|f\|_{m} \stackrel{\text { def }}{=} \sup _{\substack{\underline{\mathbf{x}} \in \mathbb{R}^{2 k} \\|\underline{\alpha}| \leq m}}\left|\left(1+|\underline{\mathbf{x}}|^{2}\right)^{m / 2}\left(D^{\underline{\alpha}} f\right)(\underline{\mathbf{x}})\right|<\infty
$$


and which vanish, together with all their partial derivatives, if at least two among the points in the set $\underline{\mathbf{x}}=\left\{\mathbf{x}_{1}, \ldots, \mathbf{x}_{k}\right\}$ are coinciding. By (99)

$$
\left|\left(S_{k, \underline{\omega}}, f\right)\right| \leq C^{k}(k !)^{3+2 \eta} \sum_{i<j} \int d \mathbf{x}_{1} \cdots d \mathbf{x}_{k} \frac{|f(\underline{\mathbf{x}})|}{\left|\mathbf{x}_{i}-\mathbf{x}_{j}\right|^{k(1+\eta) / 2-2 \varepsilon}} .
$$

On the other hand, by (202), $|f(\underline{\mathbf{x}})| \leq\|f\|_{4 k+1}\left(1+|x|^{4 k+1}\right)^{-1}$ and, for any $i \neq j,|f(\underline{\mathbf{x}})| \leq 2^{k}[(2 k) !]^{-1}\left|\mathbf{x}_{i}-\mathbf{x}_{j}\right|^{2 k}\|f\|_{2 k}$; hence, since $\|f\|_{2 k} \leq\|f\|_{4 k+1}$,

$$
|f(\underline{\mathbf{x}})| \leq\|f\|_{4 k+1} \sqrt{\left(1+|\underline{\mathbf{x}}|^{4 k+1}\right)^{-1}} \sqrt{2^{k}[(2 k) !]^{-1}\left|\mathbf{x}_{i}-\mathbf{x}_{j}\right|^{2 k}} .
$$

It follows that

$$
\left|\left(S_{k, \underline{\omega}}, f\right)\right| \leq C^{k}(k !)^{2+2 \eta}\|f\|_{4 k+1} .
$$

In order to prove the "cluster property", fixed any integer $p \in[1, k-1]$, $\mathbf{y} \in \mathbb{R}^{2}$ and $f \in \mathcal{S}_{0}\left(\mathbb{R}^{2 k}\right)$, we first prove that $\left(S_{k, \underline{\omega}}, f_{p, \mathbf{y}}\right)$ goes to 0 as $|\mathbf{y}| \rightarrow$ $\infty$, if $f_{p, \mathbf{y}}(\underline{\mathbf{x}}) \equiv f\left(\mathbf{x}_{1}, \ldots \mathbf{x}_{p}, \mathbf{x}_{p+1}-\mathbf{y}, \ldots, \mathbf{x}_{k}-\mathbf{y}\right)$. Let us consider the characteristic functions $\chi_{\mathbf{y}}(\underline{\mathbf{x}})$ and $\chi_{\mathbf{y}}^{\prime}(\underline{\mathbf{x}})$ of the set

$$
M \stackrel{\text { def }}{=}\left\{\underline{\mathbf{x}} \in \mathbb{R}^{2 k}: \max _{1 \leq j \leq p}\left|\mathbf{x}_{j}\right| \leq|\mathbf{y}| / 4, \max _{p+1 \leq j \leq k}\left|\mathbf{x}_{j}-\mathbf{y}\right| \leq|\mathbf{y}| / 4\right\}
$$

and of its complementary, respectively. Since $D_{\underline{\mathbf{x}}} \geq|\mathbf{y}| / 2$ in $M$, by using (99) and (204), we see that $\left|\left(S_{k, \underline{\omega}}, f_{p, \mathbf{y}} \chi_{\mathbf{y}}\right)\right| \leq\left[1+(|\mathbf{y}| / 2)^{2 \varepsilon}\right]^{-1} C^{k}(k !)^{2+2 \eta}\|f\|_{4 k+1}$, so that $\left(S_{k, \underline{\omega}}, f_{p, \mathbf{y}} \chi_{\mathbf{y}}\right)$ is uniformly bounded and vanishes as $|\mathbf{y}| \rightarrow \infty$. On the

other hand, by (204), $\left|\left(S_{k, \underline{\omega}}, f_{p, \mathbf{y}} \chi_{\mathbf{y}}^{\prime}\right)\right| \leq C^{k}(k !)^{2+2 \eta}\|f\|_{4 k+1} \int d \underline{\mathbf{x}} \sqrt{\left(1+|\underline{\mathbf{x}}|^{4 k+1}\right)^{-1}} \chi_{0}^{\prime}(\underline{\mathbf{x}})$, so that even $\left(S_{k, \underline{\omega}}, f_{p, \mathbf{y}} \chi_{\mathbf{y}}^{\prime}\right)$ is uniformly bounded and vanishes in the limit $|\mathbf{y}| \rightarrow \infty$, as well as $\left(S_{k, \underline{\omega}}, f_{p, \mathbf{y}}\right)$.

The cluster property E0, defined in $\S 3$ of [OS1], now simply follows, by decomposing the connected Schwinger functions as finite linear combinations of the truncated Schwinger functions, .

\section{A.2 Symmetry, Euclidean invariance and Reflection positiv- ity}

From the explicit definition of the generating functional, (13), two properties immediately follow. First, since the fields anticommute, the Schwinger functions are antisymmetric in the exchange of their arguments. Moreover, the generating functional (13) is invariant under the Lorentz transformation of the fields by construction.

Finally the "reflection positivity" E2, defined in $\S 6$ of [OS1], is verified in the lattice regularization (40), as proved in [OSe], hence it holds even in the removed cutoffs limit of the regularized model (13), which we have shown to be equivalent to the $a=0$ limit of the lattice model, see Theorem 1.4. 


\section{B Lowest order computation of $\nu_{-}$and $\nu_{+}$}

\section{B.1 Lowest order computation of $\nu_{-}$}

Calling $\widehat{g}_{\omega, \omega}(\mathbf{k}) \stackrel{\text { def }}{=} \widehat{g}_{\omega}(\mathbf{k})$ and $u_{0}(t) \stackrel{\text { def }}{=} 1-\chi_{0}(t)$, the lowest order contibution to the $\nu_{-, N}$, appearing in (113), is obtained, from (125) and (126), by taking the $\mathbf{p} \rightarrow 0$ limit of the following expression (see the first graph in Fig. 4), whose value is independent of the infrared cutoff for any fixed $\mathbf{p}$ and $|h|$ large enough:

$$
\begin{aligned}
& \lambda \int \frac{d \mathbf{k}}{(2 \pi)^{2}} \frac{C_{h, N ; \omega}(\mathbf{k}, \mathbf{k}-\mathbf{p})}{D_{-\omega}(\mathbf{p})} \widehat{g}_{\omega}^{(\leq N)}(\mathbf{k}) \widehat{g}_{\omega}^{(\leq N)}(\mathbf{k}-\mathbf{p})= \\
= & -\lambda \frac{D_{\omega}(\mathbf{p})}{D_{-\omega}(\mathbf{p})} \int \frac{d \mathbf{k}}{(2 \pi)^{2}} \frac{u_{0}\left(\gamma^{-N}|\mathbf{k}-\mathbf{p}|\right) \chi_{0}\left(\gamma^{-N}|\mathbf{k}|\right)}{D_{\omega}(\mathbf{k}-\mathbf{p}) D_{\omega}(\mathbf{k})}+ \\
& +\lambda \int \frac{d \mathbf{k}}{(2 \pi)^{2}} \frac{\chi_{0}\left(\gamma^{-N}|\mathbf{k}|\right)-\chi_{0}\left(\gamma^{-N}|\mathbf{k}-\mathbf{p}|\right)}{D_{\omega}(\mathbf{k}-\mathbf{p}) D_{-\omega}(\mathbf{p})}
\end{aligned}
$$

where we have used (111) and rearranged the terms. In the limit $|\mathbf{p}| \rightarrow 0$, the first contribution in the r.h.s. of (207) vanishes by the symmetry $\widehat{g}_{\omega}(\mathbf{k})=$ $-i \omega \widehat{g}_{\omega}\left(\mathbf{k}^{*}\right), \mathbf{k}^{*}=\left(-k_{0}, k\right)$. As regards the second term, if we write the first order Taylor expansion in $\mathbf{p}$ of the numerator as a linear combination of $D_{-\omega}(\mathbf{p})$ and $D_{\omega}(\mathbf{p})$, the term proportional to $D_{\omega}(\mathbf{p})$ also vanishes, again for the symmetry $\mathbf{k} \rightarrow \mathbf{k}^{*}$, so that

$$
\nu_{-}=-\frac{\lambda}{2} \int \frac{d \mathbf{k}}{(2 \pi)^{2}} \frac{\chi_{0}^{\prime}(|\mathbf{k}|)}{|\mathbf{k}|}=-\frac{\lambda}{4 \pi} \int_{1}^{\infty} d \rho \chi_{0}^{\prime}(\rho)=\frac{\lambda}{4 \pi} .
$$

\section{B.2 Lowest order computation of $\nu_{+}$}

If we define

$$
I_{\omega}\left(\gamma^{-N} \mathbf{k}\right)=\int \frac{d \mathbf{k}^{\prime}}{(2 \pi)^{2}} \widehat{g}_{\omega}^{(\leq N)}\left(\mathbf{k}^{\prime}\right) \widehat{g}_{\omega}^{(\leq N)}\left(\mathbf{k}^{\prime}+\mathbf{k}\right)
$$

then the lowest order contribution to the anomaly coefficient $\nu_{+, N}$, appearing in (113), is is obtained, from (125) and (126), by taking the $\mathbf{p} \rightarrow 0$ limit and, after that, the $h \rightarrow-\infty$ limit of the following expression (see the second graph in Fig. 4):

$$
\begin{aligned}
& -\lambda^{2} \int \frac{d \mathbf{k}}{(2 \pi)^{2}} \frac{C_{h, N: \omega}(\mathbf{k}, \mathbf{k}-\mathbf{p})}{D_{\omega}(\mathbf{p})} g_{\omega}^{(\leq N)}(\mathbf{k}) g_{\omega}^{(\leq N)}(\mathbf{k}-\mathbf{p}) I_{-\omega}\left(\gamma^{-N} \mathbf{k}\right)= \\
& =\lambda^{2} \int \frac{d \mathbf{k}}{(2 \pi)^{2}} \frac{u_{0}\left(\gamma^{-N}|\mathbf{k}-\mathbf{p}|\right) \chi_{0}\left(\gamma^{-N}|\mathbf{k}|\right)}{D_{\omega}(\mathbf{k}-\mathbf{p}) D_{\omega}(\mathbf{k})} I_{-\omega}\left(\gamma^{-N} \mathbf{k}\right)- \\
& -\lambda^{2} \int \frac{d \mathbf{k}}{(2 \pi)^{2}} \frac{\chi_{0}\left(\gamma^{-N}|\mathbf{k}|\right)-\chi_{0}\left(\gamma^{-N}|\mathbf{k}-\mathbf{p}|\right)}{D_{\omega}(\mathbf{k}-\mathbf{p}) D_{\omega}(\mathbf{p})} I_{-\omega}\left(\gamma^{-N} \mathbf{k}\right) .
\end{aligned}
$$


In the limit $|\mathbf{p}| \rightarrow 0$ and $h \rightarrow-\infty$, we get

$$
\nu_{+} \quad=\lambda^{2} \int \frac{d \mathbf{k}}{(2 \pi)^{2}}\left[\frac{u_{0}(|\mathbf{k}|) \chi_{0}(|\mathbf{k}|)}{|\mathbf{k}|^{4}}-\frac{\chi_{0}^{\prime}(|\mathbf{k}|)}{2|\mathbf{k}|^{3}}\right] I_{-\omega}(\mathbf{k}) D_{-\omega}^{2}(\mathbf{k})
$$

where we are using the symbol $I_{-\omega}(\mathbf{k})$ to denote even its $h=-\infty$ limit, which is finite. Note that the term in square brackets is nonnegative; moreover, it is different from 0 only for $1 \leq|\mathbf{k}| \leq \gamma_{0}$ (defined in (9)). We now fix $\omega=+$ for definiteness (the result is $\omega$-independent); then if $i k_{0}+k=y e^{i \phi}$ and $i k_{0}^{\prime}+k^{\prime}=x e^{i \vartheta}$ we get:

$$
I_{-}(\mathbf{k})=e^{-2 i \phi} \int \frac{d x d \vartheta}{(2 \pi)^{2}} \chi_{0}(x) \frac{\chi_{0}\left(\left|x e^{-i \vartheta}+y\right|\right)}{\left|x e^{-i \vartheta}+y\right|^{2}} e^{-i \vartheta}\left(x e^{-i \vartheta}+y\right),
$$

so that

$$
D_{-}^{2}(\mathbf{k}) I_{-}(\mathbf{k})=y^{2} \int \frac{d x d \vartheta}{(2 \pi)^{2}} \chi_{0}(x) \frac{\chi_{0}\left(\left|x e^{-i \vartheta}+y\right|\right)}{\left|x e^{-i \vartheta}+y\right|^{2}}(x \cos 2 \vartheta+y \cos \vartheta) .
$$

The integral (211) is easily shown to be strictly negative in the limit $\gamma_{0} \rightarrow 1$; hence by continuity in $\gamma_{0}, \nu_{+}<0$ for $\gamma_{0}-1$ small enough. Indeed in the limit $\gamma_{0} \rightarrow 1$ (211) becomes

$$
\frac{\pi \lambda^{2}}{(2 \pi)^{4}} \int_{0}^{1} d x \int_{0}^{2 \pi} d \vartheta \frac{\chi_{0}\left(\left|x e^{-i \vartheta}+1\right|\right)}{\left|x e^{-i \vartheta}+1\right|^{2}}(x \cos 2 \vartheta+\cos \vartheta)
$$

on the other hand, since $\left|x e^{-i \vartheta}+1\right| \leq 1, \cos \vartheta<0$ if $x>0$ and $x \cos 2 \vartheta+$ $\cos \vartheta=\cos \vartheta(1+x \cos \vartheta)-x \sin ^{2} \vartheta<0$ if $0<x<1$; it follows that the integrand of $(214)$ is $<0$ for $x \neq 0,1$.

A numerical calculation also shows that $\left|\nu_{+}\right|$is not constant as a function of $\gamma_{0}$, but is a strictly decreasing function near $\gamma_{0}=1$.

Acknowledgments We are indebted with K Gawedzki for enlightening discussions on the Thirring model which we have summarized in the considerations after (1.4) in the introduction. P.F. gratefully acknowledges the hospitality and the financial support of the Erwin Schrödinger Institute for Mathematical Physics (Vienna) during the preparation of this work.

\section{References}

[A] Adler S. L.: Axial-Vector Vertex in Spinor Electrodynamics. Phys. Rev. 177, 2426-2438, 1969.

[A1] Adler S. L.: Anomalies. hep-th/0411038 To appear in the Encyclopedia of Mathematical Physics, Elsevier, 2006. 
[AB] Adler S. L., Bardeen W.A.: Absence of higher order corrections in the anomalous axial vector divergence equation. Phys. Rev. 182, 1517-1536, 1969.

[AF] Akiyama A., Futami Y.: Two-fermion-loop contribution to the axial anomaly in the massive Thirring model. Phys. Rev. D 46, 798-805, 1992.

[BM1] Benfatto G., Mastropietro V.: Renormalization group, hidden symmetries and approximate Ward identities in the $X Y Z$ model. Rev. Math. Phys. 13, 1323-1435, 2001.

[BM2] Benfatto G., Mastropietro V.: On the density-density critical indices in interacting Fermi systems. Comm. Math. Phys. 231, 97$134,2002$.

[BM3] Benfatto G., Mastropietro V.: Ward identities and vanishing of the Beta function for $d=1$ interacting Fermi systems. J. Stat. Phys. 115, 143-184, 2004.

[BM4] Benfatto G., Mastropietro V.: Ward identities and chiral anomaly in the Luttinger liquid. Comm. Math. Phys. 258, 609-655, 2005.

[C] Coleman S.: Quantum sine-Gordon equation as the massive Thirring model. Phys. Rev. D 11, 2088-2097, 1975.

[CRW] Carey A.L., Ruijsenaars S.N.M., Wrigth J.D.: The massless Thirring model: Positivity of Klaiber's n-point functions. Comm. Math. Phys. 99, 347-364, 1985.

[DFZ] Dell'Antonio G., Frishman Y., Zwanziger, D.: Thirring Model in Terms of Currents: Solution and Ligth-Cone Expansions. Phys. Rev. D 6, 988-1007 1972.

[DR] Disertori M., Rivasseau, V.: Interacting Fermi Liquid in Two Dimensions at Finite Temperature. Part I: Convergent Attributions. Comm. Math. Phys. 215, 251-290, 2000.

[FGS] Furuya K., Gamboa Saravi S, Schaposnik F. A. : Path integral formulation of chiral invariant fermion models in two dimensions. Nucl. Phys. B 208, 159-181, 1982.

[FMRS] Feldman J., Magnen J., Rivasseau V, Sénéor R.: Massive GrossNeveu Model: A Rigorous Perturbative Construction. Phys. Rev. Lett 54, 1479-1481, 1985.

[G] Gallavotti G.: Renormalization theory and ultraviolet stability for scalar fields via renormalization group methods. Rev.Mod.Phys. 57, 471-562, 1985. 
[GK] Gawedzki K., Kupiainen A.: Gross-Neveu model through convergent perturbation expansions. Comm.Math.Phys. 102, 1-30, 1985.

[GL] Gomes M., Lowenstein J.H.: Asymptotic scale invariance in a massive Thirring model. Nucl. Phys. B 45, 252-266, 1972.

[GR] Georgi H., Rawls J.M.: Anomalies of the Axial-Vector Current in Two dimensions. Phys.Rev.D 3, 874-879, 1971.

[GiM] Giuliani A., Mastropietro V.: Anomalous Universality in the Ashkin-Teller model. Comm. Math. Pys 2003.

[J] Johnson K.: Solution of the Equations for the Green's Functions of a two Dimensional Relativistic Field Theory. Nuovo Cimento 20, 773-790, 1961.

[K] Klaiber B.: The Thirring model. In: Quantum theory and statistical physics. Vol X A editors Barut A.O. and Brittin. W.F. Gordon and Breach., 1968.

[Le] Lesniewski A.: Effective action for the Yukawa 2 quantum field theory. Comm. Math. Phys. 108, 437-467, 1987.

[M] Mastropietro V.: preprint 2006

[MM] Montvay I., Münster G.: Quantum Fields on a Lattice. Cambridge University Press, 1994.

[OS1] Osterwalder K., Schrader R.: Axioms for Euclidean Green's Functions. Comm. Math. Phys. 31, 83-112, 1973.

[OS2] Osterwalder K., Schrader R.: Axioms for Euclidean Green's Functions II. Comm. Math. Phys. 42, 281-305, 1975.

[OSe] Osterwalder K., Seiler E.: Gauge Field Theories on a Lattice. Ann.Phys. 110, 440-471, 1978.

[S] Seiler E: Phys. Rev. D 22, 2412-2418, 1980.

[T] Thirring W.: A soluble relativistic field theory. Ann.Phys. 3, 91$112,1958$.

[Wi] Wilson K.G.: Non-Lagrangian Models of Current Algebra. Phys. Rev. 179, 1499-1512, 1969.

[W] Wightman W.: Cargese lectures, 1976. 